Supporting Information for:

WHAT MAKES FOR A GOOD CATALYTIC CYCLE? A THEORETICAL STUDY OF THE ROLE OF ANIONIC Pd(0) COMPLEXES IN THE CROSS-COUPLING REACTION OF AN ARYL HALIDE WITH AN ANIONIC NUCLEOPHILE

By

Sebastian Kozuch ${ }^{a}$, Christian Amatore ${ }^{b}$, Anny Jutand ${ }^{b}$, Sason Shaik $^{a *}$

Contribution from [a] Department of organic Chemistry and the Lise Meitner-Minerva Center for Computational Quantum Chemistry, and [b] Ecole Normale Supérieure, Département de Chimie UMR CNRS-ENS-UPMC 8640, 24 Rue Lhomond, F-75231 Paris Cedex, France

[*] Department of Organic Chemistry, Givat Ram Campus, Jerusalem 91904, ISRAEL sason@yfaat.ch.huji.ac.il

FAX: $+972-2-6584680$ 
Table S1: Absolute and relative energies, key geometric parameters and charges of the small systems $\left(\mathrm{L}=\mathrm{PH}_{3}\right.$ and $\left.\mathrm{H}_{2} \mathrm{P}\left(\mathrm{CH}_{2}\right)_{n} \mathrm{PH}_{2}\right)$ during oxidative addition.

\begin{tabular}{|c|c|c|c|c|c|c|c|c|c|c|c|c|c|c|}
\hline & \multicolumn{2}{|c|}{ Energy } & \multicolumn{6}{|c|}{ Distances $(\AA)$} & \multirow{2}{*}{$\begin{array}{l}\text { Angles } \\
\text { P-Pd-P }\end{array}$} & \multicolumn{5}{|c|}{ Mulliken Charges } \\
\hline & Hartrees & $\mathrm{kcal} / \mathrm{mol}$ & Pd-P1 & Pd-P2 & Pd-Cl1 & $\mathrm{Pd}-\mathrm{Cl} 2$ & Pd-C & $\mathrm{Cl} 2-\mathrm{C}$ & & $\mathrm{Pd}$ & 2.L & $\mathrm{Ph}$ & Cl1 & $\mathrm{Cl} 2$ \\
\hline $\mathbf{R}_{\mathrm{H}, \mathrm{Cl}}$ & -1965.21601 & 0.00 & 2.314 & 2.279 & 2.645 & & & 1.761 & 146 & 0.151 & -0.464 & 0.019 & -0.687 & -0.019 \\
\hline $\mathrm{C}_{\mathrm{H}, \mathrm{Cl}}$ & -1965.22923 & -8.29 & 2.295 & 2.309 & 2.644 & 5.593 & 5.089 & 1.772 & 134.7 & 0.057 & -0.298 & -0.026 & -0.669 & -0.064 \\
\hline $\mathrm{TS}_{\mathrm{H}, \mathrm{Cl}}^{\mathrm{B}-\mathrm{F}}$ & -1965.21191 & 2.58 & 2.397 & 2.353 & 3.005 & & & 1.773 & 109.4 & -0.08 & 0.000 & -0.030 & -0.8 & -0.087 \\
\hline $\mathrm{TS}_{\mathrm{H}, \mathrm{Cl}}^{345}$ & -1965.21130 & 2.96 & & & & & & & & & & & & \\
\hline $\mathbf{I}_{\mathrm{H}, \mathrm{Cl}}{ }^{34}$ & -1965.21662 & -0.38 & 2.39 & 2.395 & 4.614 & 5.281 & 3.756 & 1.774 & 103.1 & -0.134 & 0.222 & -0.134 & -0.870 & -0.085 \\
\hline $\mathrm{TS}_{\mathrm{H}, \mathrm{Cl}}{ }^{234}$ & -1965.21092 & 3.20 & & & & & & & & & & & & \\
\hline $\mathbf{I}_{\mathrm{H}, \mathrm{Cl}}{ }^{23}$ & -1965.21760 & -1.00 & 2.393 & 2.393 & 4.769 & 4.272 & 3.136 & 1.771 & 103.7 & -0.113 & 0.248 & -0.190 & -0.864 & -0.080 \\
\hline $\mathrm{TS}_{\mathrm{H}, \mathrm{Cl}}{ }^{123}$ & -1965.20704 & 5.63 & & & & & & & & & & & & \\
\hline $\mathbf{I}_{\mathrm{H}, \mathrm{Cl}}{ }^{12}$ & -1965.21220 & 2.39 & 2.408 & 2.404 & 4.764 & 3.268 & 2.204 & 1.81 & 102.2 & -0.139 & 0.200 & -0.122 & -0.858 & -0.081 \\
\hline $\mathrm{TS}_{\mathrm{H}, \mathrm{Cl}}{ }^{612}$ & -1965.20445 & 7.26 & & & & & & & & & & & & \\
\hline $\mathrm{TS}_{\mathrm{H}, \mathrm{Cl}}{ }^{\mathrm{oXX}, \mathrm{Back}}$ & -1965.19276 & 14.59 & 2.459 & 2.421 & 4.824 & 2.657 & 2.108 & 2.055 & 99.9 & -0.162 & 0.310 & -0.115 & -0.845 & -0.187 \\
\hline $\mathrm{TS}_{\mathrm{H}, \mathrm{Cl}}{ }^{\text {OX,FRONT }}$ & -1965.18608 & 18.78 & 2.482 & 2.36 & 4.608 & 2.553 & 2.142 & 2.121 & 113.2 & -0.133 & 0.166 & -0.009 & -0.864 & -0.160 \\
\hline $\mathbf{I I}_{\mathrm{H}, \mathrm{Cl}}$ & -1965.25299 & -23.21 & 2.445 & 2.305 & 4.895 & 2.432 & 2.056 & & 94 & -0.243 & 0.663 & -0.231 & -0.793 & -0.396 \\
\hline $\mathbf{R}_{\mathbf{H}}$ & -1504.93100 & 0.00 & 2.305 & 2.304 & & & & & 179.9 & 0.066 & -0.066 & 0.019 & & -0.019 \\
\hline $\mathrm{C}_{\mathrm{H}}$ & -1504.93097 & 0.02 & 2.321 & 2.33 & & 4.047 & 4.078 & 1.76 & 166.8 & 0.103 & -0.067 & -0.008 & & -0.029 \\
\hline $\mathrm{TS}_{\mathrm{H}}{ }^{\mathrm{ox}}$ & -1504.89576 & 22.12 & 2.453 & 2.393 & & 2.523 & 2.161 & 2.093 & 108.7 & -0.212 & 0.330 & -0.041 & & -0.076 \\
\hline $\mathbf{I I}_{\mathrm{H}}$ & -1504.94043 & -5.91 & 2.461 & 2.307 & & 2.393 & 2.051 & 3.161 & 103.9 & -0.233 & 0.691 & -0.159 & & -0.299 \\
\hline $\mathbf{R}_{\mathbf{H}, 2}$ & -1582.33872 & 0.00 & 2.314 & 2.313 & & & & & 96 & & & 0.019 & & -0.019 \\
\hline $\mathbf{I}_{\mathrm{H}, 2}{ }^{12}$ & -1582.34668 & -5.00 & 2.38 & 2.379 & & 3.276 & 2.372 & 1.782 & 88.3 & -0.240 & 0.222 & 0.034 & & -0.017 \\
\hline $\mathbf{I}_{\mathbf{H}, 2}{ }^{23}$ & -1582.35178 & -8.20 & 2.382 & 2.371 & & & & 1.762 & 88.5 & -0.244 & 0.216 & 0.074 & & -0.045 \\
\hline $\mathbf{I}_{\mathrm{H}, 2}{ }^{34}$ & -1582.35171 & -8.15 & 2.371 & 2.369 & & & & 1.763 & 88.7 & -0.221 & 0.192 & 0.060 & & -0.031 \\
\hline $\mathrm{TS}_{\mathrm{H}, 2}{ }^{\mathrm{ox}}$ & -1582.32333 & 9.65 & 2.444 & 2.405 & & 2.521 & 2.156 & 2.082 & 85.8 & -0.205 & 0.328 & -0.044 & & -0.079 \\
\hline $\mathbf{I I}_{\mathbf{H}, 2}$ & -1582.36939 & -19.25 & 2.45 & 2.296 & & 2.385 & 2.045 & 3.16 & 85 & -0.355 & 0.702 & -0.070 & & -0.276 \\
\hline $\mathbf{R}_{\mathbf{H}, 3}$ & -1621.65779 & 0.00 & 2.291 & 2.29 & & & & & 112.8 & -0.111 & 0.111 & 0.019 & & -0.019 \\
\hline $\mathrm{C}_{\mathrm{H}, 3}$ & -1621.65799 & -0.12 & 2.303 & 2.303 & & 6.406 & 5.936 & 1.761 & 113.3 & -0.085 & 0.090 & 0.016 & & -0.022 \\
\hline $\mathbf{I}_{\mathrm{H}, 3}{ }^{12}$ & -1621.66157 & -2.37 & 2.372 & 2.358 & & 3.269 & 2.351 & 1.782 & 98.3 & -0.271 & 0.262 & 0.035 & & -0.025 \\
\hline $\mathbf{I}_{\mathrm{H}, 3}{ }^{23}$ & -1621.66747 & -6.07 & 2.355 & 2.352 & & & & 1.763 & 99.9 & -0.141 & 0.210 & -0.027 & & -0.042 \\
\hline $\mathbf{I}_{\mathbf{H}, 3}{ }^{34}$ & -1621.66632 & -5.35 & 2.355 & 2.357 & & & & 1.765 & 99.8 & -0.298 & 0.248 & 0.082 & & -0.032 \\
\hline $\mathrm{TS}_{\mathrm{H}, 3}{ }^{\mathrm{ox}}$ & -1621.63834 & 12.21 & 2.427 & 2.387 & & 2.532 & 2.166 & 2.074 & 95.3 & -0.215 & 0.348 & -0.047 & & -0.087 \\
\hline $\mathbf{I I}_{\mathbf{H}, 3}$ & -1621.68742 & -18.59 & 2.42 & 2.284 & & 2.401 & 2.05 & 3.183 & 95 & -0.275 & 0.723 & -0.147 & & -0.302 \\
\hline $\mathbf{R}_{\mathrm{H}, 4}$ & -1660.97426 & 0.00 & 2.312 & 2.31 & & & & & 127 & -0.099 & 0.099 & 0.019 & & -0.019 \\
\hline $\mathrm{C}_{\mathrm{H}, 4}$ & -1660.97696 & -1.69 & 2.315 & 2.311 & & 7.561 & 6.217 & 1.762 & 131.9 & -0.110 & 0.130 & 0.002 & & -0.022 \\
\hline $\mathbf{I}_{\mathrm{H}, 4}{ }^{23}$ & -1660.97687 & -1.63 & 2.376 & 2.36 & & & & 1.764 & 115 & -0.159 & 0.190 & 0.001 & & -0.032 \\
\hline $\mathbf{I}_{\mathrm{H}, 4}{ }^{34}$ & -1660.97686 & -1.63 & 2.354 & 2.349 & & & & 1.763 & 117.2 & -0.232 & 0.203 & 0.058 & & -0.028 \\
\hline $\mathbf{T S}_{\mathrm{H}, 4}{ }^{o x}$ & -1660.94691 & 17.16 & 2.423 & 2.379 & & 2.542 & 2.174 & 2.061 & 103 & -0.197 & 0.343 & -0.062 & & -0.084 \\
\hline $\mathbf{I}_{\mathbf{H}, 4}$ & -1660.99765 & -14.68 & 2.427 & 2.284 & & 2.403 & 2.052 & 3.162 & 100.3 & -0.269 & 0.725 & -0.150 & & -0.307 \\
\hline $\mathbf{R}_{\mathrm{H}, 5}$ & -1700.29219 & 0.00 & 2.317 & 2.316 & & & & & 144.5 & -0.061 & 0.061 & 0.019 & & -0.019 \\
\hline $\mathrm{C}_{\mathrm{H}, 5}$ & -1700.29085 & 0.84 & 2.314 & 2.314 & & 4.202 & 6.348 & 1.76 & 136.3 & -0.087 & 0.117 & -0.007 & & -0.023 \\
\hline $\mathbf{I}_{\mathrm{H}, 5}{ }^{23}$ & -1700.29115 & 0.65 & 2.368 & 2.363 & & & & 1.764 & 119.2 & -0.214 & 0.191 & 0.057 & & -0.034 \\
\hline $\mathbf{I}_{\mathbf{H}, 5^{34}}$ & -1700.29134 & 0.54 & 2.359 & 2.363 & & & & 1.764 & 119.1 & -0.215 & 0.193 & 0.051 & & -0.030 \\
\hline $\mathrm{TS}_{\mathrm{H}, 5}{ }^{\mathrm{ox}}$ & -1700.26180 & 19.08 & 2.462 & 2.412 & & 2.54 & 2.159 & 2.084 & 109.3 & -0.163 & 0.333 & -0.062 & & -0.108 \\
\hline
\end{tabular}




\begin{tabular}{|c|c|c|c|c|c|c|c|c|c|c|c|c|}
\hline $\mathbf{I I}_{\mathrm{H}, 5}$ & -1700.30878 & -10.41 & 2.439 & 2.308 & 2.4 & 2.053 & 3.132 & 102.3 & -0.261 & 0.717 & -0.151 & -0.305 \\
\hline $\mathbf{R}_{\mathrm{H}, 6}$ & -1739.60701 & 0.00 & 2.325 & 2.314 & & & & 157.2 & -0.056 & 0.056 & 0.019 & -0.019 \\
\hline $\mathbf{I}_{\mathrm{H}, 6}{ }^{23}$ & -1739.60372 & 2.06 & 2.35 & 2.351 & & & 1.761 & 128.6 & -0.180 & 0.167 & 0.042 & -0.028 \\
\hline $\mathbf{I}_{\mathbf{H}, 6}{ }^{34}$ & -1739.60318 & 2.41 & 2.351 & 2.367 & & & 1.763 & 127.5 & & & & \\
\hline $\mathbf{T S}_{\mathrm{H}, 6}{ }^{\text {ox }}$ & -1739.57265 & 21.57 & 2.471 & 2.38 & 2.548 & 2.18 & 2.09 & 116.4 & -0.202 & 0.363 & -0.055 & -0.106 \\
\hline $\mathbf{I I}_{\mathrm{H}, 6}$ & -1739.62053 & -8.48 & 2.529 & 2.316 & 2.408 & 2.036 & 3.087 & 110 & -0.277 & 0.687 & -0.116 & -0.293 \\
\hline
\end{tabular}

Table S2. Absolute, relative and zero point energies for full catalytic cycle with $\mathbf{1}_{\mathbf{H}}$.

\begin{tabular}{|c|c|c|c|c|}
\hline $1_{\mathrm{H}}$ & E (Har) & $\mathrm{E}(\mathrm{kcal} / \mathrm{mol})$ & ZPE (Har) & $\begin{array}{l}\text { ZPE } \\
(\mathrm{kcal} / \mathrm{mol})\end{array}$ \\
\hline $\mathrm{R}_{\mathrm{H}}$ & -1903.75549 & 0.00 & -1903.60433 & 0.00 \\
\hline $\mathrm{C}_{\mathrm{H}}$ & -1903.75545 & 0.02 & -1903.60408 & 0.16 \\
\hline $\mathrm{I}_{\mathrm{H}}{ }^{12}$ & Not Stable & & & \\
\hline $\mathrm{I}_{\mathrm{H}}{ }^{23}$ & Not Stable & & & \\
\hline $\mathrm{I}_{\mathrm{H}}^{34}$ & Not Stable & & & \\
\hline $\mathrm{T}_{\mathrm{H}}^{\mathrm{ox}}$ & -1903.72024 & 22.12 & -1903.57144 & 20.64 \\
\hline II & -1903.76491 & -5.91 & -1903.61288 & -5.36 \\
\hline $\mathrm{II}_{\mathrm{SH}}$ & -1903.80703 & -32.35 & -1903.65793 & -33.63 \\
\hline $\mathrm{TS}_{\mathrm{SH}}^{\mathrm{SH}-\mathrm{L}}$ & -1903.79513 & -24.88 & -1903.64296 & -24.24 \\
\hline $\mathrm{II}_{\mathrm{L}}$ & -1903.81808 & -39.28 & -1903.66712 & -39.40 \\
\hline $\mathrm{TS}_{\mathrm{SH}}^{\mathrm{L}-\mathrm{Cl}}$ & -1903.80868 & -33.38 & -1903.64296 & -24.24 \\
\hline $\mathrm{II}_{\mathrm{Cl}}^{\prime}$ & -1903.82798 & -45.49 & -1903.67499 & -44.34 \\
\hline $\mathrm{II}^{\prime}$ & -1903.78847 & -20.70 & -1903.63490 & -19.18 \\
\hline $\mathrm{TS}_{\mathrm{SH}}{ }_{\mathrm{HED}}^{\mathrm{RED}}$ & -1903.75800 & -1.58 & -1903.60640 & -1.30 \\
\hline $\mathrm{C}_{\mathrm{SH}}$ & -1903.79970 & -27.75 & -1903.64608 & -26.20 \\
\hline $\mathrm{P}$ & -1903.79235 & -23.14 & -1903.63879 & -21.62 \\
\hline
\end{tabular}


Table S3. Absolute, relative and zero point energies for full catalytic cycle with $\mathbf{1}_{\mathbf{H}, \mathbf{C l}}$.

\begin{tabular}{|c|c|c|c|c|}
\hline $1_{\mathrm{H}, \mathrm{Cl}}$ & E (Har) & $\mathrm{E}(\mathrm{kcal} / \mathrm{mol})$ & ZPE (Har) & $\begin{array}{l}\mathrm{ZPE} \\
(\mathrm{kcal} / \mathrm{mol})\end{array}$ \\
\hline $\mathrm{R}_{\mathrm{H}, \mathrm{Cl}}$ & -2364.04049 & 0.00 & -2363.89030 & 0.00 \\
\hline $\mathrm{C}_{\mathrm{H}, \mathrm{Cl}}$ & -2364.05371 & -8.29 & -2363.90392 & -8.55 \\
\hline $\mathrm{T}_{\mathrm{SH}, \mathrm{Cl}}^{\mathrm{F}-\mathrm{B}}$ & -2364.03639 & 2.58 & -2363.88727 & 1.90 \\
\hline $\mathrm{T}_{\mathrm{SH}, \mathrm{Cl}}{ }^{612}$ & -2364.02893 & 7.26 & -2363.88181 & 5.33 \\
\hline $\mathrm{I}_{\mathrm{H}, \mathrm{Cl}}^{12}$ & -2364.03669 & 2.39 & -2363.88774 & 1.61 \\
\hline $\mathrm{T}_{\mathrm{SH}, \mathrm{Cl}}{ }^{123}$ & -2364.03152 & 5.63 & -2363.88267 & 4.78 \\
\hline $\mathrm{I}_{\mathrm{H}, \mathrm{Cl}}^{23}$ & -2364.04209 & -1.00 & -2363.89216 & -1.17 \\
\hline $\mathrm{T}_{\mathrm{SH}, \mathrm{Cl}}^{234}$ & -2364.03540 & 3.20 & -2363.88558 & 2.96 \\
\hline $\mathrm{I}_{\mathrm{H}, \mathrm{Cl}}^{34}$ & -2364.04111 & -0.38 & -2363.89138 & -0.68 \\
\hline $\mathrm{T}_{\mathrm{SH}, \mathrm{Cl}}{ }^{345}$ & -2364.03578 & 2.96 & -2363.88573 & 2.86 \\
\hline $\mathrm{T}_{\mathrm{SH}, \mathrm{Cl}}{ }^{\text {OX,Back }}$ & -2364.01724 & 14.59 & -2363.86874 & 13.53 \\
\hline $\mathrm{T}_{\mathrm{SH}, \mathrm{Cl}}{ }^{\text {oX,Front }}$ & -2364.01056 & 18.78 & -2363.86275 & 17.28 \\
\hline $\mathrm{II}_{\mathrm{H}, \mathrm{Cl}}$ & -2364.07747 & -23.21 & -2363.92630 & -22.59 \\
\hline II & -2364.03637 & 2.59 & -2363.88434 & 3.74 \\
\hline $\mathrm{II}_{\mathrm{SH}}$ & -2364.07850 & -23.85 & -2363.92939 & -24.53 \\
\hline $\mathrm{TS}_{\mathrm{SH}}^{\mathrm{SH}-\mathrm{L}}$ & -2364.06659 & -16.38 & -2363.91442 & -15.14 \\
\hline $\mathrm{II}_{\mathrm{L}}$ & -2364.09244 & -32.60 & -2363.93858 & -30.30 \\
\hline $\mathrm{TS}_{\mathrm{SH}}^{\mathrm{L}-\mathrm{Cl}}$ & -2364.08015 & -24.88 & -2363.91442 & -15.14 \\
\hline $\mathrm{II}_{\mathrm{Cl}}^{\prime}$ & -2364.09944 & -36.99 & -2363.94645 & -35.24 \\
\hline II' & -2364.05993 & -12.20 & -2363.90636 & -10.08 \\
\hline $\mathrm{TS}_{\mathrm{SH}}{ }^{\mathrm{RED}}$ & -2364.02946 & 6.92 & -2363.87786 & 7.81 \\
\hline $\mathrm{C}_{\mathrm{SH}}$ & -2364.07116 & -19.24 & -2363.91755 & -17.10 \\
\hline $\mathrm{P}$ & -2364.06382 & -14.64 & -2363.91025 & -12.52 \\
\hline $\mathrm{P}_{\mathrm{Cl}}$ & -2364.07736 & -23.14 & -2363.92475 & -21.62 \\
\hline
\end{tabular}


Table S4. Absolute, relative and zero point energies for full catalytic cycle with $\mathbf{2}_{\mathbf{H}, 3}$.

\begin{tabular}{lllll}
\hline $2_{\mathrm{H}, 3}$ & $\mathrm{E}(\mathrm{Har})$ & $\mathrm{E}(\mathrm{kcal} / \mathrm{mol})$ & $\mathrm{ZPE}(\mathrm{Har})$ & $\begin{array}{l}\mathrm{ZPE} \\
(\mathrm{kcal} / \mathrm{mol})\end{array}$ \\
\hline $\mathrm{R}_{\mathrm{H}, 3}$ & -2020.48228 & 0.00 & -2020.26352 & 0.00 \\
$\mathrm{C}_{\mathrm{H}, 3}$ & -2020.48247 & -0.12 & -2020.26357 & -0.03 \\
$\mathrm{I}_{\mathrm{H}, 3}^{12}$ & -2020.48605 & -2.37 & -2020.26699 & -2.17 \\
$\mathrm{I}_{\mathrm{H}, 3}^{23}$ & -2020.49195 & -6.07 & -2020.27236 & -5.55 \\
$\mathrm{I}_{\mathrm{H}, 3}{ }^{34}$ & -2020.49080 & -5.35 & -2020.27182 & -5.21 \\
$\mathrm{~T}_{\mathrm{SH}, 3}{ }^{\circ}$ & -2020.46282 & 12.21 & -2020.24471 & 11.81 \\
$\mathrm{II}_{3}$ & -2020.51190 & -18.59 & -2020.29171 & -17.69 \\
$\mathrm{II}_{\mathrm{SH}, 3}$ & -2020.55067 & -42.92 & -2020.32802 & -40.47 \\
$\mathrm{TS}_{\mathrm{SH}, 3}{ }_{\mathrm{SH}-\mathrm{Cl}}$ & -2020.53880 & -35.47 & -2020.31754 & -33.89 \\
$\mathrm{II}_{\mathrm{Cl}, 3}^{\prime}$ & -2020.57149 & -55.98 & -2020.34939 & -53.88 \\
$\mathrm{II}_{3}^{\prime}$ & -2020.53618 & -33.82 & -2020.31369 & -31.48 \\
$\mathrm{TS}_{\mathrm{SH}, 3}{ }^{\mathrm{RED}}$ & -2020.50035 & -11.34 & -2020.27921 & -9.85 \\
$\mathrm{C}_{\mathrm{SH}, 3}$ & Not Stable & & -2020.29798 & -21.62 \\
$\mathrm{P}_{3}$ & -2020.51914 & -23.14 & & \\
\hline & & & & \\
\hline
\end{tabular}

Table S5. Absolute, relative and zero point energies for full catalytic cycle with $\mathbf{2}_{\mathbf{H}, \mathbf{6}}$.

\begin{tabular}{lllll}
\hline $2_{\mathrm{H}, 6}$ & $\mathrm{E}(\mathrm{Har})$ & $\mathrm{E}(\mathrm{kcal} / \mathrm{mol})$ & $\mathrm{ZPE}(\mathrm{Har})$ & $\begin{array}{l}\mathrm{ZPE} \\
(\mathrm{kcal} / \mathrm{mol})\end{array}$ \\
\hline $\mathrm{R}_{\mathrm{H}, 6}$ & -2138.43150 & 0.00 & -2138.12650 & 0.00 \\
$\mathrm{C}_{\mathrm{H}, 6}$ & -2138.43393 & -1.53 & -2138.12862 & -1.33 \\
$\mathrm{I}_{\mathrm{H}, 6}^{12}$ & Not Stable & & & \\
$\mathrm{I}_{\mathrm{H}, 6}^{23}$ & -2138.42821 & 2.06 & -2138.12353 & 1.86 \\
$\mathrm{I}_{\mathrm{H}, 6}^{34}$ & -2138.42766 & 2.41 & -2138.12279 & 2.33 \\
$\mathrm{~T}_{\mathrm{SH}, 6}$ ox & -2138.39713 & 21.57 & -2138.09324 & 20.87 \\
$\mathrm{II}_{6}$ & -2138.44502 & -8.48 & -2138.13858 & -7.58
\end{tabular}




\begin{tabular}{lllll}
$\mathrm{II}_{\mathrm{SH}, 6}$ & -2138.48851 & -35.78 & -2138.18191 & -34.77 \\
$\mathrm{TS}_{\mathrm{SH}, 6}{ }_{\mathrm{SH}-\mathrm{Cl}}$ & -2138.46651 & -21.97 & -2138.15967 & -20.82 \\
$\mathrm{II}_{\mathrm{Cl}, 6}$ & -2138.50586 & -46.67 & -2138.19785 & -44.77 \\
$\mathrm{II}_{6}^{\prime}$ & -2138.46966 & -23.95 & -2138.16152 & -21.97 \\
$\mathrm{TS}_{\mathrm{SH}, 6}{ }_{\mathrm{RED}}$ & -2138.43515 & -2.29 & -2138.12850 & -1.25 \\
$\mathrm{C}_{\mathrm{SH}, 6}$ & -2138.47104 & -24.81 & -2138.16309 & -22.96 \\
$\mathrm{P}_{6}$ & -2138.46836 & -23.14 & -2138.16096 & -21.62 \\
\hline
\end{tabular}

Table S6. Absolute, relative and zero point energies for oxidative addition with $\mathbf{2}_{\mathbf{H}, \mathbf{2}}$.

\begin{tabular}{lllll}
\hline $2_{\mathrm{H}, 2}$ & $\mathrm{E}(\mathrm{Har})$ & $\mathrm{E}(\mathrm{kcal} / \mathrm{mol})$ & $\mathrm{ZPE}(\mathrm{Har})$ & $\begin{array}{l}\mathrm{ZPE} \\
(\mathrm{kcal} / \mathrm{mol})\end{array}$ \\
\hline $\mathrm{R}_{\mathrm{H}, 2}$ & -1582.33872 & 0.00 & -1582.15413 & 0.00 \\
$\mathrm{C}_{\mathrm{H}, 2}$ & Not Stable & & & \\
$\mathrm{I}_{\mathrm{H}, 2}^{12}$ & -1582.34668 & -5.00 & -1582.16233 & -5.15 \\
$\mathrm{I}_{\mathrm{H}, 2}^{23}$ & -1582.35178 & -8.20 & -1582.16732 & -8.28 \\
$\mathrm{I}_{\mathrm{H}, 2}^{34}$ & -1582.35171 & -8.15 & -1582.16709 & -8.14 \\
$\mathrm{~T}_{\mathrm{SH}, 2}$ ox & -1582.32333 & 9.65 & -1582.13966 & 9.07 \\
$\mathrm{II}_{2}$ & -1582.36939 & -19.25 & -1582.18355 & -18.47 \\
\hline
\end{tabular}

Table S7. Absolute, relative and zero point energies for oxidative addition with $\mathbf{2}_{\mathbf{H , 4}}$.

\begin{tabular}{lllll}
\hline $2_{\mathrm{H}, 4}$ & $\mathrm{E}(\mathrm{Har})$ & $\begin{array}{l}\mathrm{E} \\
(\mathrm{kcal} / \mathrm{mol})\end{array}$ & $\mathrm{ZPE}(\mathrm{Har})$ & $\begin{array}{l}\mathrm{ZPE} \\
(\mathrm{kcal} / \mathrm{mol})\end{array}$ \\
\hline $\mathrm{R}_{\mathrm{H}, 4}$ & -1660.97426 & 0.00 & -1660.73201 & 0.00 \\
$\mathrm{C}_{\mathrm{H}, 4}$ & -1660.97696 & -1.69 & -1660.73459 & -1.62 \\
$\mathrm{I}_{\mathrm{H}, 4}^{12}$ & Not Stable & & & \\
$\mathrm{I}_{\mathrm{H}, 4}{ }^{23}$ & -1660.97687 & -1.63 & -1660.73568 & -2.30 \\
$\mathrm{I}_{\mathrm{H}, 4}{ }^{34}$ & -1660.97686 & -1.63 & -1660.73526 & -2.04
\end{tabular}




\begin{tabular}{lllll}
$\mathrm{T}_{\mathrm{SH}, 4}$ ox & -1660.94691 & 17.16 & -1660.70656 & 15.97 \\
$\mathrm{II}_{4}$ & -1660.99765 & -14.68 & -1660.75471 & -14.24 \\
\hline
\end{tabular}

Table S8. Absolute, relative and zero point energies for oxidative addition with $\mathbf{2}_{\mathbf{H , 5}}$.

\begin{tabular}{lllll}
\hline $2_{\mathrm{H}, 5}$ & $\mathrm{E}(\mathrm{Har})$ & $\begin{array}{l}\mathrm{E} \\
(\mathrm{kcal} / \mathrm{mol})\end{array}$ & $\mathrm{ZPE}(\mathrm{Har})$ & $\begin{array}{l}\mathrm{ZPE} \\
(\mathrm{kcal} / \mathrm{mol})\end{array}$ \\
\hline $\mathrm{R}_{\mathrm{H}, 5}$ & -1700.29219 & 0.00 & -1700.02156 & 0.00 \\
$\mathrm{C}_{\mathrm{H}, 5}$ & -1700.29085 & 0.84 & -1700.02018 & 0.87 \\
$\mathrm{I}_{\mathrm{H}, 5}{ }^{12}$ & Not Stable & & & \\
$\mathrm{I}_{\mathrm{H}, 5}{ }^{23}$ & -1700.29115 & 0.65 & -1700.02127 & 0.18 \\
$\mathrm{I}_{\mathrm{H}, 5}{ }^{34}$ & -1700.29134 & 0.54 & -1700.02102 & 0.34 \\
$\mathrm{~T}_{\mathrm{SH}, 5}{ }^{\circ}$ & -1700.26180 & 19.08 & -1699.99230 & 18.36 \\
$\mathrm{II}_{5}$ & -1700.30878 & -10.41 & -1700.03660 & -9.44 \\
\hline
\end{tabular}

Table S9. Solvation energy of $\mathrm{Cl}^{-}$and $\mathbf{T} \mathbf{S}_{\mathbf{H}, \mathbf{C l}}^{\text {ox,back }}$ with explicit $\mathrm{THF}$ molecules and polarizable continuum model.

\begin{tabular}{cc}
\hline \multicolumn{3}{c}{ Cl $^{-}$with $\boldsymbol{n}$ THF } \\
Number of THF & Solv. E $(\mathrm{kcal} / \mathrm{mol})^{\mathrm{a}}$ \\
\hline 4 & $21.94(71.5)$ \\
7 & $34.19(71.5)$ \\
9 & $34.72(71.5)$ \\
10 & $34.31(71.7)$ \\
\hline continuum & 136.04 \\
\hline Transition State with $\boldsymbol{n}$ $\mathbf{~ H F}$ \\
Number of THF & Solv. E $(\mathrm{kcal} / \mathrm{mol})$ \\
\hline 5 & 35.86 \\
6 & 37.69 \\
7 & 34.68 \\
7 - opt & 38.64 \\
8 & 39.50 \\
\hline
\end{tabular}

${ }^{a}$ Data in parentheses corresponds to $\mathrm{Cl}^{-}(\mathrm{THF})_{\mathrm{n}}$ embedded in a continuum with the dielectric constant of THF.

${ }^{b}$ In all entries the geometry of $\mathbf{T S H}_{, \mathbf{C l}}{ }^{\text {ox,Back }}$ was kept fixed, except for the entry "7-opt" where the position of $\mathrm{Cl}^{-}$was

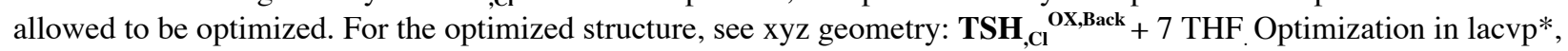
single point in lacvp*+. 


\section{XYZ Structures:}

$1_{\mathrm{H}}$

$\begin{array}{lr}\text { Geometry: } \\ \text { Pd } & 0.0180941520 \\ \text { P } & -0.0270900554 \\ \text { P } & 0.0674374739 \\ \text { H } & -1.1315007626 \\ \text { H } & 0.6924603275 \\ \text { H } & 0.6933998563 \\ \text { H } & -1.2560689329 \\ \text { H } & 0.5671125945 \\ \text { H } & 0.5669512345\end{array}$

$1_{\mathrm{H}, \mathrm{Cl}}$

Geometry:

$\begin{array}{lr}\mathrm{Pd} & 0.0357298749 \\ \mathrm{P} & -0.0832728429 \\ \mathrm{P} & 1.4268507838 \\ \mathrm{H} & 1.0635508528 \\ \mathrm{H} & 2.8226048951 \\ \mathrm{H} & 1.5412275937 \\ \mathrm{H} & -1.2216130436 \\ \mathrm{H} & -0.1332903595 \\ \mathrm{H} & 0.8374895314 \\ \mathrm{Cl} & -2.2992216764\end{array}$

68 Total energy:

0.0122057375

0.0459737697

0.0530793902

0.8461841498

0.3620031247

$-1.1483690651$

0.5712543366

$-1.2044485766$

0.6182859948

$-0.3119939053$

$\mathrm{C}_{\mathrm{H}, \mathrm{Cl}}$

\begin{tabular}{lr}
\multicolumn{2}{c}{22} \\
Geometry: \\
P & 1.0026383019 \\
Pd & 0.6101578361 \\
$\mathrm{Cl}$ & 2.6995149448 \\
$\mathrm{P}$ & -1.1893500301 \\
$\mathrm{H}$ & -1.4441606348 \\
$\mathrm{H}$ & -2.5685832643 \\
$\mathrm{H}$ & -1.2869682341 \\
$\mathrm{H}$ & 1.9486282686 \\
$\mathrm{H}$ & 1.5140807809 \\
$\mathrm{H}$ & 0.0126648868 \\
$\mathrm{C}$ & -0.9338559853 \\
$\mathrm{C}$ & -0.1175214277 \\
$\mathrm{C}$ & 1.0241084756 \\
$\mathrm{C}$ & 1.3290040260 \\
$\mathrm{C}$ & 0.4978846541 \\
$\mathrm{C}$ & -0.6456184205 \\
$\mathrm{H}$ & -0.3465414728 \\
$\mathrm{H}$ & 1.6574406844 \\
$\mathrm{H}$ & 2.2168233233 \\
$\mathrm{H}$ & 0.7329567290 \\
$\mathrm{H}$ & -1.3001297271 \\
$\mathrm{Cl}$ & -2.3829551705
\end{tabular}

62 Total energy:

0.1992230990

$-0.4982928279$

$-0.3182484153$

$-1.4356733528$

$-1.2781315062$

$-1.2731522251$

$-2.8596918413$

1.2040837415

$-0.7538892830$

0.6854134150

4.0754493727

2.9486527042

2.8728762465

3. 9183493322

5.0412146271

5.1289841854

2.1307644283

1. 9877263809

3.8543133135

5.8560291423

5.9940858759

4.1886632476
$-813.084331739233$

$-0.0004714940$

2.3039108789

$-2.3039800000$

$-3.0618691611$

$-3.0199423136$

$-3.0180448807$

3. 0121868458

3. 0457168940

3. 0455460290

$-1273.36933989174$

0.0543141114

2.3299212880

$-1.7946179993$

$-2.9265029959$

$-1.9318023316$

$-2.5580175404$

3. 0211976601

3.0196787485

3.2709592578

$-1.1448302966$

$-1965.22922872770$

$-0.3312105022$

1.8195214282

3. 4291266850

2. 9208043906

4.3204605176

2.5744362430

2. 9736607334

$-0.7081552191$

$-1.2667576178$

$-1.2440072202$

3. 4300247152

3.3396693229

4.1476573809

5.0261501353

5.1020767778

4. 3010544574

2. 6604123722

4.0717349418

5.6514365160

5.7848007906

4. 3491115221

2. 4158474882

\section{$\mathrm{T}_{\mathrm{SH}, \mathrm{Cl}}{ }^{\mathrm{F}-\mathrm{B}}$}

$\begin{array}{lcrrr}\text { Geometry: } & 10 & \text { Total } & \text { energy: } & -1965.21190786359 \\ \mathrm{C} & 0.0367363426 & -0.0345243902 & 0.0591958049 \\ \mathrm{C} & 0.0879677311 & -0.0380916807 & 1.4838239614 \\ \mathrm{C} & 1.2667630421 & -0.0059154718 & -0.6413818301 \\ \mathrm{X} & 0.0618928675 & -0.0362760630 & 0.7587414295 \\ \mathrm{Pd} & -0.7064408666 & 2.0554268389 & 0.7725122476 \\ \mathrm{P} & -1.1293971597 & 3.2625487067 & -1.2541342382 \\ \mathrm{P} & -1.3847931424 & 3.3557933263 & 2.6123583168 \\ \mathrm{Cl} & -3.6052936127 & 1.6679258452 & 0.0819747735 \\ \mathrm{H} & -0.6459040961 & 3.8524825117 & 3.7381489766 \\ \mathrm{H} & -2.0064665572 & 4.6130428376 & 2.3555920770 \\ \mathrm{H} & -2.4623435851 & 2.8638733703 & 3.4025397116 \\ \mathrm{H} & -1.7610236704 & 2.5859934486 & -2.3228286580 \\ \mathrm{H} & -2.0991892444 & 4.2879807337 & -1.1398291546 \\ \mathrm{H} & -0.2441933815 & 4.0263521550 & -2.0909834988 \\ \mathrm{C} & 1.3448239189 & 0.0241502190 & 2.1378628892 \\ \mathrm{C} & 2.5242269095 & 0.0725649348 & 1.4098260182\end{array}$




$\begin{array}{lrrr}\mathrm{C} & 2.4926161845 & 0.0510159008 & -0.0037542839 \\ \mathrm{H} & -0.8857752608 & -0.2745084929 & -0.4599440148 \\ \mathrm{H} & -0.8049017643 & -0.2679799173 & 2.0561918828 \\ \mathrm{H} & 1.3738666280 & 0.0206413250 & 3.2253629576 \\ \mathrm{H} & 3.4835319656 & 0.1244310981 & 1.9198819122 \\ \mathrm{H} & 3.4103304831 & 0.0826817656 & -0.5826250320 \\ \mathrm{Cl} & 1.2204058813 & -0.0570363311 & -2.4126937147\end{array}$

\section{$\mathrm{I}_{\mathrm{H}, \mathrm{Cl}}{ }^{12}$}

$\begin{array}{lrrrr}\text { Geometry: } & 12 & \text { Total } & \text { energy: } & -1965.21220384947 \\ \mathrm{Pd} & 0.0395454627 & -0.0113457178 & -0.0107373720 \\ \mathrm{C} & 0.0113718586 & -0.0030570334 & 2.1933949897 \\ \mathrm{Cl} & 1.7188444998 & -0.0103185422 & 2.7927302877 \\ \mathrm{P} & -0.5117927274 & -1.1576555514 & -2.0556737190 \\ \mathrm{P} & 1.1192954482 & 1.9787277054 & -0.8196539714 \\ \mathrm{H} & 1.0484240796 & 2.2293404322 & -2.2183591597 \\ \mathrm{H} & 0.7813561836 & 3.2761393963 & -0.3420640712 \\ \mathrm{H} & 2.5224498515 & 2.1306348930 & -0.6544715681 \\ \mathrm{H} & -1.8240884168 & -1.6458834416 & -2.3101491084 \\ \mathrm{H} & -0.3002268527 & -0.4374743835 & -3.2655647868 \\ \mathrm{H} & 0.1521609573 & -2.3637022381 & -2.4141288871 \\ \mathrm{C} & -0.8367318521 & 1.0183557198 & 2.7230943920 \\ \mathrm{C} & -2.2027199267 & 0.8283501416 & 2.7772266837 \\ \mathrm{C} & -2.7830543268 & -0.4028500538 & 2.3664737635 \\ \mathrm{C} & -1.9773580658 & -1.4165158557 & 1.8874676402 \\ \mathrm{C} & -0.5643816737 & -1.2489845206 & 1.7693696038 \\ \mathrm{H} & -0.3858416453 & 1.9442005139 & 3.0678530789 \\ \mathrm{H} & -2.8392980474 & 1.6313262752 & 3.1416841259 \\ \mathrm{H} & -3.8586655962 & -0.5443353451 & 2.4365192218 \\ \mathrm{H} & -2.4087850880 & -2.3734798302 & 1.6007160652 \\ \mathrm{H} & 0.0681255399 & -2.1246845878 & 1.6569474835 \\ \mathrm{Cl} & 0.5487568803 & 1.4628865284 & -4.5122328312 \\ \mathrm{X} & -0.2877305172 & -0.6252638785 & 1.9896989375 \\ & & & \end{array}$

\section{$\mathbf{I}_{\mathrm{H}, \mathrm{Cl}}{ }^{23}$}

1 HCl.PhCl. int-C23Pd.PS.sp.xyz

$\begin{array}{rrrr}\mathrm{Pd} & 0.00000 & 0.00000 & 0.00000 \\ \mathrm{C} & 2.28313 & 0.00000 & 0.00000 \\ \mathrm{C} & 2.85378 & 1.30100 & 0.00000 \\ \mathrm{Cl} & 3.49336 & 1.92474 & -1.52960 \\ \mathrm{P} & -0.82135 & 0.85023 & -2.08031 \\ \mathrm{Cl} & -4.56124 & 0.46511 & -1.31392 \\ \mathrm{P} & -1.98262 & -0.65855 & 1.16716 \\ \mathrm{H} & -2.28477 & -0.21156 & 2.48613 \\ \mathrm{H} & -3.22584 & -0.34084 & 0.54758 \\ \mathrm{H} & -2.24263 & -2.03348 & 1.43603 \\ \mathrm{H} & -0.49121 & 0.26318 & -3.33489 \\ \mathrm{H} & -2.23715 & 0.85813 & -2.22069 \\ \mathrm{H} & -0.56580 & 2.18809 & -2.48816 \\ \mathrm{C} & 1.81791 & -0.52157 & 1.25106 \\ \mathrm{C} & 1.91917 & 0.28246 & 2.42253 \\ \mathrm{C} & 2.45217 & 1.55661 & 2.36226 \\ \mathrm{C} & 2.93488 & 2.07969 & 1.13317 \\ \mathrm{H} & 2.47069 & -0.65873 & -0.84138 \\ \mathrm{H} & 1.64415 & -1.58972 & 1.35465 \\ \mathrm{H} & 1.56832 & -0.12070 & 3.36952 \\ \mathrm{H} & 2.51001 & 2.17122 & 3.25738 \\ \mathrm{H} & 3.36163 & 3.07640 & 1.08504\end{array}$

$I_{\mathrm{H}, \mathrm{Cl}}{ }^{34}$

$\begin{array}{lrrrr}\text { Geometry: } & 23 & \text { Total } & \text { energy: } & -1965.21662464236 \\ \text { Pd } & -0.0458322059 & 0.0149662168 & 0.0356291751 \\ \text { C } & -0.0313165869 & -0.0051566193 & 2.3309430095 \\ \text { C } & 2.4003691917 & 0.0509452833 & 1.9923017359 \\ \text { C } & 1.1331179465 & 0.6889608127 & 1.8791561636 \\ \text { C } & 0.1077339071 & -1.3131721387 & 2.8807029526 \\ \text { C } & 2.5216296645 & -1.2246854767 & 2.5115085141 \\ \text { C } & 1.3548087385 & -1.8932956405 & 2.9520476613 \\ \text { Cl } & 1.5244918230 & -3.5260631701 & 3.6251483028 \\ \text { P } & -2.1552116525 & -0.7672113225 & -0.7701771677 \\ \text { Cl } & -2.2607708869 & 1.7096658132 & -3.6404079872 \\ \text { P } & 0.9934232806 & 0.8964833348 & -1.9334078853 \\ \text { H } & 1.6771231774 & 0.0517390851 & -2.8514768014 \\ \text { H } & 0.0668834528 & 1.4713472944 & -2.8484927943\end{array}$


1.9830495175
-3.3423388212
-2.6763665520
-2.2872210460
-0.7677958065
-0.9581743501
1.0956869383
3.2905080857
3.4882022337

$\mathrm{T}_{\mathrm{SH}, \mathrm{Cl}}{ }^{123}$

Geometry:

Pd $\quad 0.0334925506$

$-0.0957872835$

1. 1408925120

2.1391862770

1. 9107263736

0.6782007201

$-0.3474966916$

$\mathrm{Cl} \quad-1.4345789048$

1.3069590195

3. 1011140243

2. 6934066472

0.4811385093

$-1.3572624408$

0.1926145337

$-0.0694293662$

1. 2011339351

0.3325653747

$-0.8791110046$

0.8335169355

0.0878998390

$-1.2374410126$

$\mathrm{Cl} \quad 0.3583820658$

\section{$\mathrm{T}_{\mathrm{SH}, \mathrm{Cl}}{ }^{234}$}

Geometry:

Pd $\quad-0.0528142235$

C $\quad 0.0504485063$

1. 4355591786

2.2323056836

1.6438061399

0.2430043021

$-0.5665146953$

$\mathrm{Cl} \quad-0.9598771929$

$\mathrm{H} \quad 1.8856339674$

3. 3143003256

2. 2579586550

$-0.2252653668$

$-1.6468110816$

1.3088605964

$-2.0431905627$

2. 6567222256

0.7371553195

1. 5059653245

$-2.4522106798$

$-2.0268468094$

$-3.3462510211$

Cl -1.0439560804

\section{$\mathrm{T}_{\mathrm{SH}, \mathrm{Cl}}{ }^{345}$}

$\begin{array}{lr}\text { Geometry: } \\ \text { Pd } & -0.1042552765 \\ \text { C } & 0.0551883854 \\ \text { C } & 2.1071435940 \\ \text { C } & 0.7662915359 \\ \text { C } & 0.6744950006 \\ \text { C } & 2.7338443814 \\ \text { C } & 2.0061513784 \\ \text { Cl } & 2.7960768619 \\ \text { P } & -2.2601011246 \\ \text { Cl } & -1.5557179827 \\ \text { P } & 1.0241140167 \\ \text { H } & 2.3844881155\end{array}$

1. 9230555117

$-0.8958648330$

0.0306513856

$-2.0344222146$

$-1.8419706869$

0.5330847554

1. 7594614097

0.5822626927

$-1.7123485701$

9 Total energy:

$-0.0540964937$

0.0848429132

$-0.0862933868$

0.8694784214

2.0028655173

2.1686706538

1. 2082612229

$-0.9821901746$

$-0.9612253853$

0.7317039712

2. 7449082173

3. 0439762097

1. 4083155314

1.1557230063

$-2.3747526881$

2. 1045901866

0.2686146965

1. 9424321874

$-3.3778757770$

$-2.6082768310$

$-3.1662698010$

$-1.7700069934$

34 Total energy:

0.0176328109

0.0022859270

$-0.0435186585$

0.7443417786

1.5622955005

1. 5967261706

0.8068840988

$-0.9924871689$

$-0.6877154660$

0.7013385253

2. 1814139112

2.3194173366

0.8614087215

$-0.2030157135$

$-1.2532321204$

$-0.6705007839$

$-1.1612980074$

0.8302362448

$-2.3440831807$

$-1.9366462222$

$-0.6850107890$

$-2.6520253055$

5 Total energy: 0.3751189016 $-0.7817799697$ 0.2328838770 0.3647547805 $-2.0394382354$ $-1.0211043998$ $-2.1356441264$ $-3.7183478818$ $-0.4100833130$ $-0.0052625175$

1. 2966677711

1.0740627031
$-1.9506926822$

0.0094838451

$-1.8266097922$

$-1.4030327878$

3. 2441117568

2.5062674325

1.6991486495

1.6639193812

2.5844704894

$-1965.20704321352$

0.0438287121

2. 7958981828

3. 4400121604

3. 2630714494

2. 4644743149

1.8348397596

1. 9827388984

3. 2532380113

4. 0602190141

3. 7510031719

2.3315740058

1.2228899898

1. 6334579313

$-2.0062604153$

$-0.4650717548$

$-2.3526190897$

$-3.1217124381$

$-2.5214640367$

$-0.0027135611$

$-1.8614115661$

$-0.2722455745$

$-4.2818953120$

$-1965.21091730461$

$-0.0756950672$

3. 5021889689

3. 6425892156

2.8004660823

1.8322060770

1. 6932612611

2.5363388959

4. 5601266827

4.3914588073

2. 8933521762

1.1855500194

1.0272786491

2. 4745614714

$-2.0050327398$

$-0.3823235680$

$-2.0633291420$

$-2.8926610566$

$-2.9671920446$

0.4366821456

$-1.6285053499$

$-0.4918179126$

$-3.7758028456$

$-1965.21130288787$

0.0962489004

2. 6984582098

1. 8821852452

2. 2948937732

2.7049113510

1. 8823699755

2. 2994550089

2. 3158133687

$-0.4978659450$

$-4.2183704363$

$-1.7751618871$

$-2.1535437984$ 


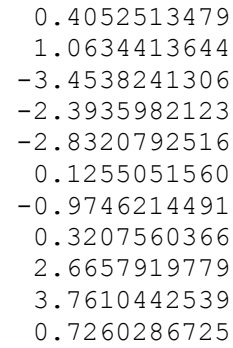

31 Total energy: 0.0067405457 0.0037148478

0.0195209364

$-0.9539762797$

1. 7831489597

2. 0170387453

1. 6854988089

3.1238975219

$-2.2606499842$

$-0.2488990318$

$-1.1123651772$

1.0896587509

0.8901919506

$-0.3849951466$

$-1.4681136302$

$-1.2960088206$

2. 0751063259

1. 7404065065

$-0.5258794331$

$-2.4650707019$

$-2.1468375116$

1. 3825865365
$-2.9971051286$

$-2.0262571489$

$-0.2530652328$

$-1.9114697657$

$-0.1457681663$

2. 9998641097

3. 0286174323

2. 4113762206

1. 5824009425

1.5496846949

2. 2955147216

$-1965.20454914908$

$-0.0210447417$

2. 0193147321

2. 7525241408

3.1803769272

2. 8591617311

2.0998008321

1. 6751744244

1. 8559213652

3.0231279465

3. 7372827216

3.1826251660

1. 8200085895

1.1280995228

$-0.3716159205$

$-2.0789950068$

0.4906490613

$-1.5333430460$

$-0.7072950006$

$-2.2630205044$

$-2.9599034907$

$-2.9976476696$

$-3.6383795976$

$-1965.19276136457$

$-0.0028205041$

2.1052743906

1.7384978169

$-2.2584521922$

$-0.4347460710$

$-1.7829913524$

0.1570635907

$-0.0224153245$

$-2.5315483312$

$-3.2629732265$

$-2.9470903604$

2.8716694429

3. 7320751779

3. 8911949424

3.1918193436

2. 3313997044

2.7380976235

4.2769748816

4.5669905629

3. 3258222798

1.8382965272

$-4.1287801516$

\section{$\mathbf{T}_{\mathrm{SH}, \mathrm{Cl}}$ ox, Front}

23

Geometry: 8 Total energy: $\quad-1965.18607789498$

C $\quad-0.0049826722$

Cl $\quad-0.0037582019$

C $\quad 1.2592593101$

1. 3957302614

0.3223502741

$-0.9151473984$

$-1.0847043692$

2.1184539286

2. 3631439278

0.4456616691

$\begin{array}{rr}-0.0078714704 & -0.0184238325 \\ -0.0139329352 & 2.1027724888 \\ 0.0031863134 & -0.6252863079 \\ 0.6478682283 & -1.8640307956 \\ 1.3274987807 & -2.4470641148 \\ 1.3611101099 & -1.7842769557 \\ 0.7109227384 & -0.5609646735 \\ -0.5106088062 & -0.1951001900 \\ 0.6054526178 & -2.3581015882 \\ 1.8358770404 & -3.4007425825\end{array}$




$\begin{array}{lr}\mathrm{H} & -1.7577827640 \\ \mathrm{H} & -2.0381013416 \\ \mathrm{X} & -0.0044054200 \\ \mathrm{Pd} & -0.1798824697 \\ \mathrm{P} & -1.6595955076 \\ \mathrm{C} 1 & 4.2802797255 \\ \mathrm{P} & 1.4437092040 \\ \mathrm{H} & 1.3745353664 \\ \mathrm{H} & 2.6906940088 \\ \mathrm{H} & 1.9193873950 \\ \mathrm{H} & -1.1765149727 \\ \mathrm{H} & -2.1158746722 \\ \mathrm{H} & -2.9278024503\end{array}$

\section{$I I_{H, C l}$}

$\begin{array}{lr}\text { Geometry: } \\ \text { Pd } & 0.0060143638 \\ \mathrm{C} & 0.0236129454 \\ \mathrm{Cl} & 2.4371386111 \\ \mathrm{P} & 0.0267806456 \\ \mathrm{P} & -2.2907485669 \\ \mathrm{H} & -3.0588880638 \\ \mathrm{H} & -2.7789200375 \\ \mathrm{H} & -2.9295754376 \\ \mathrm{H} & 0.6011748779 \\ \mathrm{H} & -1.1946613456 \\ \mathrm{H} & 0.8243668294 \\ \mathrm{C} & -0.5193485048 \\ \mathrm{C} & -0.4817396553 \\ \mathrm{C} & 0.0854746096 \\ \mathrm{C} & 0.6278278587 \\ \mathrm{C} & 0.6000026659 \\ \mathrm{H} & -0.9858366076 \\ \mathrm{H} & -0.9051656075 \\ \mathrm{H} & 0.1091509374 \\ \mathrm{H} & 1.0832672801 \\ \mathrm{H} & 1.0350106820 \\ \mathrm{Cl} & -3.7917817937\end{array}$

\section{$\mathrm{C}_{\mathrm{H}}$}

1.8912890423

0.7338406838 $-0.0107290273$ $-2.0564789987$ $-2.7923235068$ $-2.3158830234$ $-3.7963279048$ $-5.1740815568$ $-3.4809710063$ $-4.0000582888$ $-2.6961162117$ $-4.1352450048$ $-2.2063711848$

5 Total energy: 0.0059035664 0.0133683443 $-0.0075946609$ $-0.0160577118$ $-0.1242961700$ $-0.0849480279$ $-1.2720250268$ 0.8543776480 $-1.1692028040$ 0.0899989703 0.9630585460 1.0703231052 1. 0501828267 $-0.0422382143$ $-1.0974335203$ $-1.0670693232$ 1. 9119921590 1.8844337624 $-0.0706868575$ $-1.9439888472$ $-1.8907893345$ 0.2314431851

Total energy:

0.0003326025

0.0049441374

0.0973965594

1. 4263165925

$-1.0036842489$

$-1.1991941104$

$-2.2942525142$

$-0.3510425248$

1. 4476568356

2. 8260374452

1. 4134227570

1. 1201849445

1.0375049958

$-0.1482692829$

$-1.2589387096$

$-1.1887537075$

2.0336317467

1. 9040542676

$-0.2068799994$

$-2.1855505770$

$-2.0460812494$
$-2.2251105814$

$-0.0426430747$

0.9815719251

0.5818217013

$-1.1035534687$

$-0.5471119475$

1. 2871611208

0.9423981268

0.6472821256

2. 6102551420

$-2.4349595221$

$-1.2343433256$

$-1.3924556478$

$-1965.25299287607$

$-0.0183549002$

2.0371697020

$-0.0786182617$

$-2.4630010103$

0.1222947793

$-1.0974673430$

0.7871957725

0.9132820056

$-3.0586381903$

$-3.1939130648$

$-3.1034480587$

2. 7924662134

4. 2043511617

4. 8800591903

4.1314312355

2. 7281442188

2.2847986516

4.7685165541

5.9742599585

4.6393013755

2.1654264348

$-3.0991749685$

\footnotetext{
$-1504.93096525816$

$-0.0508764584$

4.0194135131

3. 7114663419

$-0.3014740064$

0.0408350113

$-1.1656492747$

0.5765130021

0.7386413041

$-1.4847125969$

$-0.2517025714$

0.6106461549

3.7742321167

4.0296966212

4.5163550567

4. 7456231326

4.4984582178

3.3886515992

3. 8429329077

4. 7155145786

5.1196301334

4. 6718542148
}

$\mathrm{T}_{\mathrm{H}}$ ox

Geometry:

Pd $\quad 0.0083768872$

56 Total energy:

0.0412346751

$-1504.89575925605$

0.0024100544

2.1635447519

1. 5440030647

0.5591502922

$-0.8373156605$

0.7838365816

0.9603352164

0.0252388925

$-2.1298188325$

$-0.3070841060$

$-1.5655052663$

0.2775168545

0.2389661556

2. 0298629621

$-2.4459731893$ 


\begin{abstract}
0.3661766374
2. 2206208434

$-0.6592886262$

$-1.5472451337$

$-1.7568563476$

$-1.0507823763$

$-0.1446239738$

$-0.4847872116$

$-2.0791538323$

$-2.4435028069$

$-1.1818207190$

0.4439536692
\end{abstract}

\section{II}

Geometry:

$\begin{array}{lr}\mathrm{Pd} & 0.0229637570 \\ \mathrm{C} & 0.0047204701 \\ \mathrm{Cl} & 2.4160739404 \\ \mathrm{P} & 0.3269661715 \\ \mathrm{P} & -2.2675432811 \\ \mathrm{H} & -3.1504016762 \\ \mathrm{H} & -2.8362825508 \\ \mathrm{H} & -2.8319462398 \\ \mathrm{H} & -0.6891147453 \\ \mathrm{H} & 1.0971359717 \\ \mathrm{H} & 1.0923826765 \\ \mathrm{C} & 0.0133071828 \\ \mathrm{C} & 0.0640020148 \\ \mathrm{C} & 0.1022833641 \\ \mathrm{C} & 0.0916848549 \\ \mathrm{C} & 0.0412084638 \\ \mathrm{H} & 0.0015427375 \\ \mathrm{H} & 0.0809134364 \\ \mathrm{H} & 0.1448816632 \\ \mathrm{H} & 0.1299522614 \\ \mathrm{H} & 0.0517253090\end{array}$

\section{$I_{\mathrm{SH}}$}

Geometry:

$\mathrm{Pd} \quad 0.4301261166$

C $\quad-0.6948519605$

C $\quad-0.5247115173$

-0.5247115173
0.1127478294

0.5679907155

0.3924990506

$-0.2404588667$

0.7914357852

$-1.7694247690$

2.7470091810

$-2.6709941615$

$-2.0568385967$

$-2.3902189456$

0.3047907654

0.3559372919

2.1478015940

1. 0704377245

0.7554995414

$-0.3775276321$

$-1.1878345557$

$-0.8902731153$

$-2.9306029161$

$-4.0154759051$

\section{$\mathrm{TS}_{\mathrm{SH}}^{\mathrm{SH}-\mathrm{L}}$}

Geometry:

$\mathrm{Pd} \quad-0.0217959426$

C $\quad-0.0196155593$

C1 2.4759087495

P $\quad 0.2147371243$

P $\quad-1.9670325785$

$\mathrm{H} \quad-2.9697223961$

$-2.8317172039$

$-2.0782464204$

$-0.8625537425$
$-0.3620864018$

$-0.7592012686$

1.0926117599

0.7445592499

$-0.6004721963$

$-1.5999355671$

$-1.2735122575$

2.1327192621

1.5314365749

$-0.8587776903$

$-2.6435432136$

$-2.0454991217$
$-3.3784893629$

$-2.4681713193$

2. 8718529262

3. 8887026856

4. 2337255485

3. 5597356807

2. 5422329461

2. 6160456797

4.4188309979

5.0348857460

3.8362540686

2.0570201266
28 Total energy:

$-0.0062428513$

$-0.0024161797$

$-0.0194720442$

$-0.0037427760$

0.0011331629

0.0008817424

$-1.0658945802$

1.0752139599

0.0015352107

1.0657009100

$-1.0741175098$

1.2097100821

1. 2119564961

0.0054633958

$-1.2049997202$

$-1.2107859763$

2. 1567247350

2. 1590767183

0.0086278876

$-2.1489190661$

$-2.1607332904$

$-1504.94042619830$

$-0.0005923216$

2. 0503863587

0.0067075987

$-2.4432383867$

0.2726813783

$-0.8340936297$

0.9984626801

0.9929150354

$-3.4368291183$

$-2.9459100826$

$-2.9524798455$

2. 7488049516

4.1495720195

4.8555372458

4.1560515246

2.7552478693

2. 2145219579

4. 6845958985

5.9418184520

4.6964631259

2.2258972443

56 Total energy: 0.1655598947 $-1.3741514691$

$-1.1965426472$

$-0.0546517206$

0.9121256403

0.7416557395

$-0.4024866707$

0.3811673356

0.8855497213

$-0.5888180404$

$-0.0212598915$

2. 0069857372

1.1453783960

$-0.6170312206$

1. 4951281882

0.3686593466

1. 8050872738

1.5048705966

$-0.5347908435$

$-2.2718978780$

$-1.9640216732$

1. 1907523002

0.3987571175

$-1903.80703317212$

0.1067242634

4.0032014146

2. 6233215005

2. 1125615361

3. 0223071792

4. 4024650847

4.9006382596

$-2.3288804738$

$-0.0024398568$

0.3201830409

0.5993679530

0.8166765985

$-1.3885600335$

$-3.2015955910$

$-3.0828451812$

$-2.7271483894$

2. 6592415429

5.0894740313

5.9722040111

4. 3740035670

1. 9448723426

$-3.2659581926$

$-3.0837135044$

20 Total energy:

$-0.0655783125$

$-0.0710630272$

0.2051184806

$-0.3153947490$

1.3973629443

1. 3881954410

1.0059549541

2. 8001871939

$-0.4421995460$

$-1903.79512853575$

$-0.0082192393$

2.0270391133

$-0.0931571986$

$-2.3936453842$

0.1498844117

$-0.8634035619$

1.2009212298

0.4121201787

$-3.3059830654$ 


\begin{abstract}
0.9858092647
0.9189992739

0.6481634475

0.6654842164

0.0242924544

$-0.6334631787$

$-0.6616544852$

1.1829512923

1.1911433341

0.0398292600

$-1.1300276391$

$-1.1713258905$

$-1.5266817013$

$-2.6504718169$
\end{abstract}

\section{$I I_{L}$}

Geometry:

$\begin{array}{lr}\mathrm{Pd} & -0.1281622389 \\ \mathrm{C} & -0.1083002313 \\ \mathrm{Cl} & 2.2143179810 \\ \mathrm{P} & 0.0189743987 \\ \mathrm{P} & 0.3630830118 \\ \mathrm{H} & -0.8839474859 \\ \mathrm{H} & 1.0962706351 \\ \mathrm{H} & 0.6292930935 \\ \mathrm{H} & -0.9989438456 \\ \mathrm{H} & 1.0984510571 \\ \mathrm{H} & 0.2094959741 \\ \mathrm{C} & 0.9429131385 \\ \mathrm{C} & 1.0149856941 \\ \mathrm{C} & 0.0422298074 \\ \mathrm{C} & -1.0047969033 \\ \mathrm{C} & -1.0825437872 \\ \mathrm{H} & 1.7261097842 \\ \mathrm{H} & 1.8407852774 \\ \mathrm{H} & 0.1012948117 \\ \mathrm{H} & -1.7719211899 \\ \mathrm{H} & -1.9200150333 \\ \mathrm{~S} & -2.3865680949 \\ \mathrm{H} & -2.7207396273\end{array}$

\section{$\mathrm{TS}_{\mathrm{SH}}^{\mathrm{L}-\mathrm{Cl}}$}

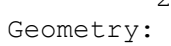

$\begin{array}{lrr}\mathrm{Pd} & 0.0042685705 & -0.0818201654 \\ \mathrm{C} & 0.0115827104 & -0.0688104158 \\ \mathrm{Cl} & 2.7656311153 & 0.1392227131 \\ \mathrm{P} & 0.1701205253 & -0.2752696133 \\ \mathrm{P} & 0.6660180310 & 2.5801514354 \\ \mathrm{H} & -0.0709142157 & 3.7703864097 \\ \mathrm{H} & 1.6113242205 & 2.7337091489 \\ \mathrm{H} & 1.4584745570 & 3.2021334858 \\ \mathrm{H} & -0.9605424193 & -0.4520346289 \\ \mathrm{H} & 0.8094718500 & 0.7164575778 \\ \mathrm{H} & 0.9116001100 & -1.3800785605 \\ \mathrm{C} & 1.1721995554 & -0.4942567438 \\ \mathrm{C} & 1.2453994546 & -0.4540871658 \\ \mathrm{C} & 0.1676084888 & 0.0220457903 \\ \mathrm{C} & -0.9869538811 & 0.4587763013 \\ \mathrm{C} & -1.0660574996 & 0.4056073572 \\ \mathrm{H} & 2.0260591532 & -0.8366805057 \\ \mathrm{H} & 2.1522547297 & -0.7944503460 \\ \mathrm{H} & 0.2272079818 & 0.0528521707 \\ \mathrm{H} & -1.8340481747 & 0.8344649546 \\ \mathrm{H} & -1.9789233136 & 0.7241921140 \\ \mathrm{~S} & -1.9218512315 & -1.5090535109 \\ \mathrm{H} & -1.7989897980 & -2.0274388375\end{array}$

\section{II' $\mathrm{Cl}$}

$\begin{array}{ll}\text { Geometry: } \\ \text { C } & 2.7796672002 \\ \text { C } & 2.0524695758 \\ \text { C } & 2.7704817752 \\ \text { C } & 4.1694082150 \\ \text { C } & 4.8809382771\end{array}$

\author{
0.6115145817 \\ $-1.4803663334$ \\ 0.9588797974 \\ 0.9981694925 \\ $-0.0003614633$ \\ $-1.0336675208$ \\ $-1.0691905295$ \\ 1. 7199277968 \\ 1.8055417103 \\ 0.0268467811 \\ $-1.8213997314$ \\ $-1.8775305206$ \\ $-2.3488277001$ \\ $-1.6492537936$
}

26 Total energy: $-0.0919618937$

$-0.0912936927$

$-0.8783564696$

$-0.1143923154$

3. 5126493370

3. 7882905112

3.5363285003

4.8903687977

0.3810935357

0.5770789002

$-1.3489080434$

0.5535773161

0.5428309505

$-0.1238573302$

$-0.7710652718$

$-0.7498493823$

1.0539541461

1.0511203185

$-0.1389354260$

$-1.2895817864$

$-1.2386615315$

0.6689919856

0.5934626658
32 Total energy: $-0.0311642572$

0.0210590607

$-0.0129751200$

$-0.1440801535$
$-0.0933307708$
$-3.1591415346$

$-2.7495302499$

2. 7117310478

4. 1125753508

4.8541002707

4.1780814779

2.7741411453

2.1503059160

4. 6222905660

5.9427650467

4. 7437281333

2.2569143397

$-0.5977227720$

$-0.8920467461$

$-1903.81807797853$

0.0531821441

2.0916658308

$-0.0938494869$

$-2.3501703650$

$-0.7168206425$

$-0.1023040929$

0.4996848455

$-1.0218879370$

$-3.2164601142$

$-2.9441777416$

$-3.0219764612$

2. 7708662534

4.1705470385

4.9259997205

4. 2627006044

2. 8624055081

2.2045966304

4. 6701209471

6.0133757210

4.8341564619

2. 3713611297

0.1843371432

$-1.1246303818$

$-1903.80868473279$

0.0057853460

2. 0279658242

$-0.5190383521$

$-2.4066292699$

0.0954927794

0.4187447748

1. 1362116420

$-0.9054249367$

$-3.2565891133$

$-3.1891774573$

$-2.8745441565$

2. 6951784952

4.0946380571

4.8508872307

4.1925899615

2.7936621519

2. 1170716205

4.5931675887

5.9374825772

4. 7659880088

2. 2973444555

0.0556458809

1. 2966010114

$-1903.82797920471$

$-1.2043513730$

$-0.0033644899$

1. 2038690467

1. 2124103353

0.0088693107 


$\begin{array}{lr}\mathrm{C} & 4.1786199125 \\ \mathrm{Pd} & 0.0058909430 \\ \mathrm{P} & 0.0634652032 \\ \mathrm{~S} & 0.1551225075 \\ \mathrm{P} & -2.4298372113 \\ \mathrm{H} & -1.1503728281 \\ \mathrm{H} & 0.7694467071 \\ \mathrm{H} & 0.7854733958 \\ \mathrm{H} & -3.1984686498 \\ \mathrm{H} & -3.0409654471 \\ \mathrm{H} & -3.0439187361 \\ \mathrm{H} & 2.2365515581 \\ \mathrm{H} & 4.7015530749 \\ \mathrm{H} & 5.9670320597 \\ \mathrm{H} & 4.7181374530 \\ \mathrm{H} & 2.2528555560 \\ \mathrm{H} & 1.4984694668 \\ \mathrm{Cl} & -3.4073164012\end{array}$

\section{II'}

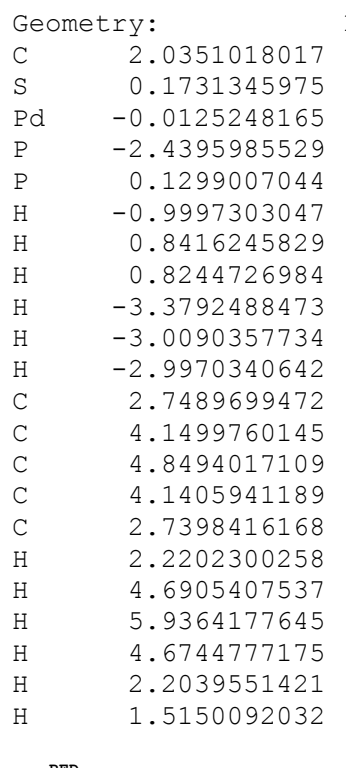

\section{$\mathrm{TS}_{\mathrm{SH}}{ }^{\mathrm{RED}}$}

$\begin{array}{lr}\text { Geometry: } \\ \text { C } & 0.0022615919 \\ \text { S } & 0.0009187414 \\ \text { Pd } & 1.9715847322 \\ \text { P } & 3.7683463933 \\ \text { P } & 3.0797591612 \\ \text { H } & 4.4702280805 \\ \text { H } & 2.6534110521 \\ \text { H } & 2.8864206713 \\ \text { H } & 4.9703071728 \\ \text { H } & 3.4140389813 \\ \text { H } & 4.3932687636 \\ \mathrm{C} & -0.2474083192 \\ \mathrm{C} & -0.9413094743 \\ \mathrm{C} & -1.4331322553 \\ \mathrm{C} & -1.2181067050 \\ \mathrm{C} & -0.5095925613 \\ \mathrm{H} & 0.0843850214 \\ \mathrm{H} & -1.1100023164 \\ \mathrm{H} & -1.9837601560 \\ \mathrm{H} & -1.5937756940 \\ \mathrm{H} & -0.3457051442 \\ \mathrm{H} & -0.4603502313\end{array}$

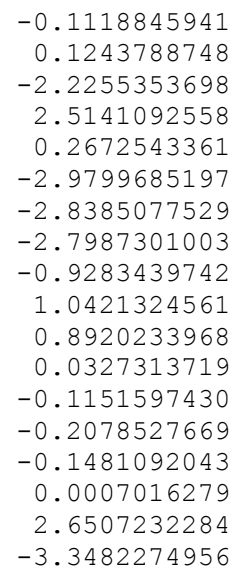

$-0.0094390988$ 2. 4146563762 0.0444553978 0.3515575611 $-2.3002943598$ $-3.1593642015$ $-2.9264058488$ $-2.8804234584$ $-0.7088101651$ 1.1527582674 1.0259392757 $-0.0082541304$ $-0.0244115511$ $-0.0412477448$ $-0.0440915415$ $-0.0290159928$ 0.0321374941 $-0.0120369706$ $-0.0455838630$ $-0.0471225137$ $-0.0041179753$ 2. 5454372450

$-1.2007309185$

$-0.0115992905$

0.0279678417

$-0.0436591568$

$-0.0192799900$

0.0541289304

$-1.0331944691$

1.1000867529

0.0630648070

0.9948107276

$-1.1306625287$

2.1505018367

2. 1623527326

0.0135492610

$-2.1460507222$

$-2.1554640561$

$-0.0475273504$

0.1534692325

$-1443.51700403818$

$-0.0016085039$

$-0.0472477712$

0.0029399640

$-0.0186957836$

0.0558228723

0.0638458610

$-0.9908562567$

1.1441098917

0.0366353178

0.9963393585

$-1.1276728693$

1. 2045652159

1.1988563998

$-0.0114710869$

$-1.2169432231$

$-1.2128565679$

2. 1536332100

2.1428110115

$-0.0158058478$

$-2.1647714424$

$-2.1584402079$

$-0.0668757056$

4 Total energy:

0.0006032431

0.0001927858

$-0.0005293454$

0.8353880843

$-0.9198644135$

$-1.1554413123$

$-2.1929365064$

$-0.2493256283$

1. 4935649485

1. 7571741605

$-0.0989489714$

1. 2628057416

1. 3624687240

0.2195207743

$-1.0337597320$

$-1.1486581304$

2.1664837309

2. 3448714724

0.3021130652

$-1.9334444847$

$-2.1329995215$

$-1.2641386423$

$-1443.48653524130$

$-0.0017666057$

2. 2009618785

0.8119695504

2. 2829796184

$-1.1337413187$

$-1.3130939860$

$-1.5916971654$

$-2.3672142140$

1. 9020522332

3.3003874456

3. 1478427768

$-0.5796348527$

$-1.7901566498$

$-2.4286707985$

$-1.8395448036$

$-0.6419735098$

$-0.0760617329$

$-2.2260526248$

$-3.3617541027$

$-2.3227877533$

$-0.2101747767$

2.1103540165

\section{$\mathrm{C}_{\mathrm{SH}}$}

22

Geometry: 23 Total energy: -1443.52823778263

$\begin{array}{llll}\mathrm{Pd} & 0.0218121138 & -0.0523999082 & -0.0253677238\end{array}$

C $\quad 4.2762188173 \quad 0.0824877346 \quad 0.1732668261$

S $\quad 3.2727307910 \quad 1.5454392663 \quad 0.3779760302$ 


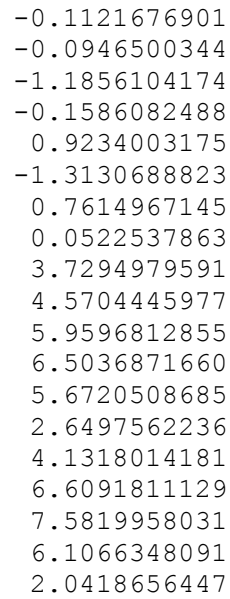

6 Total energy: 0.2729252767 0.2472396251 0.0364402534 0.7855007431 $-1.2310580860$ $-0.5571651984$ 1. 4424451106 $-0.2947816534$ 0.3157067155 $-0.0557436739$ $-1.3888378961$ 1. 4038997581 $-0.0761684594$
$-2.3004276706$

2. 2452526982

3. 0235228683

2. 6696268645

3. 1445253755

$-2.8377050866$

$-2.9145154514$

$-3.2557457342$

$-0.0369966828$

$-0.1855268297$

$-0.1281831486$

0.0813594597

0.2310030204

$-0.0840162984$

$-0.3466848438$

$-0.2447775329$

0.1285574574

0.3944373554

0.2170495585

$-890.492043434123$

$-0.2037066130$

0.2406054616

$-2.4966743685$

$-3.6487400420$

$-3.1394740700$

1.0093338276

0.3801290197

$-1.4684096976$

$-2.6326724278$

$-1.5919285549$

$-1.4876646381$

$-2.6553356316$

$-3.5855720829$

$2_{\mathrm{H}, 3}$

\begin{tabular}{lr}
\multicolumn{3}{c}{16} \\
Geometry: \\
Pd & -0.0903519886 \\
P & -2.3220227215 \\
P & 0.3279669794 \\
H & -3.2056947447 \\
H & -2.9083459458 \\
H & 0.6630847274 \\
H & 1.0987288929 \\
C & -3.1866377066 \\
C & -1.3521686337 \\
C & -2.5476314260 \\
H & -1.2941026045 \\
H & -1.5245813941 \\
H & -2.2765253942 \\
H & -3.3367688319 \\
H & -3.2040928351 \\
H & -4.2314411704
\end{tabular}

$2_{\mathrm{H}, 4}$

Geometry:

Pd $\quad 0.0196790671$

P $\quad-2.2532580962$

1. 1738385987

1. 4642550353

2. 4072442317

$-3.1961429697$

$-2.6971798010$

$-3.1581355666$

$-2.2373443016$

$-1.6059427432$

$-2.8553147689$

$-3.5698492985$

$-4.0119254228$

0.0650296775

0.5364785930

0.0504771697

$-1.3605923984$
38 Total energy:

$-0.0072323038$

0.1399816510

0.1097449522

$-0.6315709360$

1. 3921917652

1. 3483977573

$-0.6862909870$

$-0.1468494628$

$-0.1662952369$

0.5492522569

0.1429605512

$-1.2504619472$

1. 5842418074

0.6288030177

$-1.2327611403$

0.1737941645

39 Total energy: $-0.5214852545$ $-0.2827188121$ $-0.1835567774$ 1.1692366057 $-0.6858967192$ $-1.0527649569$ 0.9699264366 $-0.2610651984$ 0.1329319340 0.9759630968 0.5075986745 $-1.2641315655$ 0.4218583262 $-0.6530612902$ $-1.5106311044$ 0.1637423653 $-1.0374496247$
$-929.811121446157$

$-0.1514412247$

0.3449811296

$-2.3999788663$

1.1572865139

0.6825876004

$-3.0159106265$

$-3.2989082168$

$-1.3029504828$

$-3.2039575542$

$-2.5292757680$

$-4.2545509787$

$-3.2050302294$

$-2.2779822241$

$-3.2898672061$

$-1.4630266096$

$-1.2120434833$

$-969.127590006470$

$-0.0537884695$

0.2847264359

$-2.0285555006$

$-2.3499275240$

$-2.5360064986$

1.0257289617

0.7899708172

$-1.3744257195$

$-2.5479932627$

$-2.2385631745$

$-3.3759991378$

$-1.5433605217$

$-1.2999741399$

$-3.4882921457$

$-3.9830261050$

$-4.2199983643$

$-3.0551954222$ 


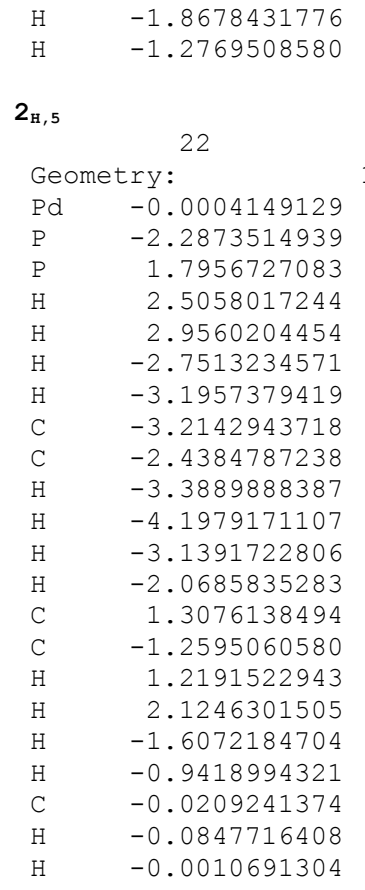

\section{$2_{\mathrm{H}, 6}$}

\begin{tabular}{lr}
\multicolumn{2}{c}{25} \\
Geometry \\
Pd & 0.0004747657 \\
$\mathrm{P}$ & 0.0043966887 \\
$\mathrm{P}$ & 0.8977349136 \\
$\mathrm{C}$ & 2.7080874813 \\
$\mathrm{C}$ & 3.5429938372 \\
$\mathrm{C}$ & 3.3050217214 \\
$\mathrm{C}$ & 3.4431507630 \\
$\mathrm{C}$ & 2.8896185363 \\
$\mathrm{H}$ & -0.9736308036 \\
$\mathrm{H}$ & -0.1496125058 \\
$\mathrm{H}$ & 0.9787998536 \\
$\mathrm{H}$ & 0.4189662740 \\
$\mathrm{H}$ & 2.7493164649 \\
$\mathrm{H}$ & 3.1101805291 \\
$\mathrm{H}$ & 4.6062581589 \\
$\mathrm{H}$ & 3.3160239708 \\
$\mathrm{H}$ & 2.8509161856 \\
$\mathrm{H}$ & 3.5921247828 \\
$\mathrm{H}$ & 3.9902839858 \\
$\mathrm{H}$ & 2.2866703374 \\
$\mathrm{H}$ & 4.4997982498 \\
$\mathrm{H}$ & 2.9166137231 \\
$\mathrm{C}$ & 1.5171862343 \\
$\mathrm{H}$ & 1.5289443816 \\
$\mathrm{H}$ & 1.3512411002
\end{tabular}

\section{$I_{\mathrm{H}, 2}{ }^{12}$}

$\begin{array}{lr}\text { Geometry: } \\ \text { C } & -0.9963451098 \\ \text { C } & -0.3294776271 \\ \text { C } & 0.9053891619 \\ \text { C } & 1.4660470645 \\ \text { C } & 0.7811275627 \\ \text { C } & -0.4579283456 \\ \text { Cl } & 1.9846116855 \\ \text { Pd } & -0.8050602662 \\ \text { C } & -2.9814748545 \\ \text { P } & -2.9167584567 \\ \text { C } & -1.6079363658 \\ \text { P } & -0.2487992474 \\ \text { H } & 0.8591940341 \\ \text { H } & -0.3554239557 \\ \text { H } & -4.2726576387 \\ \text { H } & -3.1205381814\end{array}$

$-1.5220711875$

$-1.7974178813$

12 Total energy:

$-0.2171487047$

0.1032970922

$-0.1941860032$

1.0271535370

$-1.0180849082$

1. 4089107172

$-0.5714286953$

$-0.1430947929$

0.3969722043

$-1.2208165199$

0.3335105412

0.5175893720

1. 4058476336

$-0.4722065193$

$-0.5120448765$

$-1.5562905265$

$-0.1263716520$

$-1.2594512256$

$-1.0723478157$

0.2211408499

0.2945935826

1. 2557878300
$-3.9020523624$

$-2.2683861195$

$-1008.44552192071$

$-0.0524899069$

0.1321818013

$-1.5150891003$

$-1.6699811822$

$-1.4828103431$

0.4482316113

0.9958533116

$-1.4887218588$

$-2.7093373759$

$-1.6032966834$

$-1.4080128123$

$-3.5471963268$

$-2.4813983348$

$-3.3127721598$

$-3.1230523795$

$-3.4614632215$

$-3.9563533755$

$-3.8506970427$

$-2.2347081148$

$-3.6850939767$

$-4.7795777390$

$-3.3166114769$

$-1047.76034576150$

$-0.0002792996$

2.3134474857

$-2.1446274052$

$-2.2118347746$

$-1.0893457980$

0.3011568198

1.4666300261

2.8190927801

3. 0937584524

2.9567941487

$-2.8525711676$

$-3.2448080536$

$-2.1247440881$

$-3.1975016785$

$-1.3572294705$

$-1.0547484251$

2.8139413993

3. 6202333633

0.4496073503

0.3288183508

1. 5858659679

1.1999887472

3. 2422961829

3. 1262338198

4. 3073297744
8 Total energy: 0.6911602627 1. 5658464000 2.1350127721 1. 8085471683 0.9622808335 0.3991479815 2.8302189564 3. 7650561600 6.3326438562 4. 6732430596 7.0269705592 5.8783341341 6.7446745190 6.1367637947 4. 2875630972 5.1309563019
$-1582.34668146071$

0.8515451204

1. 7522051986

1. 3409488341

0.0795790822

$-0.7760307590$

$-0.3906177637$

2. 5770161039

1.1360147031

0.8536440371

1.7471349941

0.8172542118

0.1932673354

0.4063300961

$-1.2028055844$

1. 5349311462

3. 0797959430 
-3.7268775001
-3.3216952182
-1.3146996449
-1.6603806593
2.4306811917
1.2068228361
-0.9775418463
-1.9310807725
-0.6312450070

\section{$I_{H, 2} 23$}

Geometry:

$\begin{array}{lr}\mathrm{C} & 0.0330295459 \\ \mathrm{Cl} & 0.0537079333 \\ \mathrm{Pd} & 2.8217192440 \\ \mathrm{C} & 5.8953449934 \\ \mathrm{P} & 4.2694355417 \\ \mathrm{C} & 5.6779963212 \\ \mathrm{P} & 4.4695174394 \\ \mathrm{H} & 4.4309774473 \\ \mathrm{H} & 5.3830625403 \\ \mathrm{H} & 4.8059223648 \\ \mathrm{H} & 4.0534767841 \\ \mathrm{H} & 6.5236598878 \\ \mathrm{H} & 6.4267569636 \\ \mathrm{H} & 5.2432434978 \\ \mathrm{H} & 6.6377953506 \\ \mathrm{C} & -0.7323666269 \\ \mathrm{C} & -0.7566594877 \\ \mathrm{C} & -0.0279909779 \\ \mathrm{C} & 0.7631146214 \\ \mathrm{C} & 0.8074762822 \\ \mathrm{H} & -1.3125309378 \\ \mathrm{H} & -1.3650860757 \\ \mathrm{H} & -0.0684350521 \\ \mathrm{H} & 1.1562633129 \\ \mathrm{H} & 1.2133238178\end{array}$

31 Total energy: 0.0475590762

0.1247207886

0.0688641634

$-1.0474116811$

$-0.7037719573$

$-1.6075979067$

$-0.5656287472$

$-1.3810877034$

0.3850125516

$-0.1068478054$

$-1.9820406896$

$-1.7301534632$

$-0.0882061836$

$-2.6140757375$

$-1.7004217639$

$-0.9101651690$

$-0.9451234543$

$-0.0310195077$

0.9536155994

0.9932673695

$-1.6201482229$

$-1.6938650258$

$-0.0483518106$

1. 7885322275

1. 8538243474
1. 3111979516

$-0.1688084147$

1.8311348087

0.2106244164

$-0.1940352062$

$-1.7474423766$

$-1.0634080259$

1.1676496881

2. 7934830249

\section{$I_{\mathrm{H}, 2}{ }^{34}$}

Geometry: 8 Total energy: $\quad-1582.35171166926$

$\begin{array}{lrrr}\mathrm{C} & -0.0127726894 & -0.0045535708 & -0.0504610257 \\ \mathrm{Pd} & 0.0507226074 & 0.0063903177 & 3.1270650604 \\ \mathrm{C} & 2.4158628653 & -0.0357903997 & 5.5270401776 \\ \mathrm{P} & 2.3275660628 & -0.3716666954 & 3.6720429694 \\ \mathrm{C} & 1.4962964194 & 1.1233855655 & 5.9515899643 \\ \mathrm{P} & -0.2601719256 & 0.9192470772 & 5.2904267608 \\ \mathrm{H} & -0.7902763846 & 2.1522865771 & 5.7659920069 \\ \mathrm{H} & -0.8033469960 & 0.1313343175 & 6.3436459396 \\ \mathrm{H} & 3.1779191785 & -1.5131927302 & 3.6408363108 \\ \mathrm{H} & 3.3230500358 & 0.5429916717 & 3.2291807804 \\ \mathrm{H} & 3.4471169063 & 0.1658484456 & 5.8381842250 \\ \mathrm{H} & 2.1033968940 & -0.9631920796 & 6.0248699519 \\ \mathrm{H} & 1.8729604609 & 2.0696812336 & 5.5416622950 \\ \mathrm{H} & 1.4923152091 & 1.2231699340 & 7.0428953310 \\ \mathrm{C} & -0.4300344614 & 1.2655038993 & -0.3971987756 \\ \mathrm{C} & -1.5236906503 & 1.8881893004 & 0.2420687875 \\ \mathrm{C} & -2.1939481935 & 1.2072465116 & 1.2440861671 \\ \mathrm{C} & -1.7914426016 & -0.0951901144 & 1.6384832009 \\ \mathrm{C} & -0.6884847605 & -0.7035656738 & 0.9842773816 \\ \mathrm{H} & -1.8321734552 & 2.8835943681 & -0.0614517108 \\ \mathrm{H} & -3.0488285010 & 1.6710912215 & 1.7296336552 \\ \mathrm{H} & -2.4375873258 & -0.6828314175 & 2.2844315848 \\ \mathrm{Cl} & 0.4105079648 & 2.1401127552 & -1.6765479863 \\ \mathrm{H} & -0.4804337632 & -1.7605494964 & 1.1251830076 \\ \mathrm{H} & 0.8197638156 & -0.4703093157 & -0.5685541770 \\ \mathrm{X} & -1.2409838418 & -0.3848978603 & 1.2996880162 \\ & & & \end{array}$

\section{$\mathbf{T}_{\mathrm{SH}, 2}$ ox}

$$
25
$$

$\begin{array}{llrrr}\text { Geometry: } & 6 \text { Total } & \text { energy: } & -1582.32333289078 \\ \mathrm{C} & 0.0026549214 & 0.0093383979 & 0.0056043059 \\ \mathrm{Cl} & 0.0043395509 & 0.0133352117 & 2.0877731925 \\ \mathrm{Pd} & 2.0646192299 & -0.0082511360 & 0.6342747787 \\ \mathrm{C} & 5.5006048035 & 0.1449110085 & 0.3924234646 \\ \mathrm{P} & 4.2375106849 & -0.8672054703 & 1.3494585457\end{array}$



5.0225385377
3. 2928470250
3. 0785270728
3. 6197539885
4. 8993332077
4.6334527371
6.4673849077
5. 6497673561
4.9713383277
5.7358158600
$-0.1851543132$
$-0.8385597518$
$-1.3574944839$
$-1.2269077412$
$-0.5690114239$
0.1644320223
$-0.9607211553$
$-1.8818652244$
$-1.6432259006$
$-0.4742089007$

$\mathrm{II}_{2}$

Geometry:

$\begin{array}{lr}\text { C } & -3.3270451747 \\ \text { P } & -2.8208323335 \\ \text { Pd } & -0.3764268316 \\ \text { C } & 1.6583925433 \\ \text { C } & -2.2957457427 \\ \text { P } & -0.5481263236 \\ \text { Cl } & -0.2047892102 \\ \mathrm{H} & 0.2329941089 \\ \mathrm{H} & -0.3251734249 \\ \mathrm{H} & -3.7138902131 \\ \mathrm{H} & -3.4500439925 \\ \mathrm{H} & -4.3282561556 \\ \mathrm{H} & -3.3702738702 \\ \mathrm{H} & -2.3503276500 \\ \mathrm{H} & -2.5028853400 \\ \mathrm{C} & 2.4310069293 \\ \mathrm{C} & 3.8281274626 \\ \mathrm{C} & 4.4606026056 \\ \mathrm{C} & 3.6893104276 \\ \mathrm{C} & 2.2911419965 \\ \mathrm{H} & 1.9581141349 \\ \mathrm{H} & 4.4200516145 \\ \mathrm{H} & 5.5446402201 \\ \mathrm{H} & 4.1712286776 \\ \mathrm{H} & 1.7093572835\end{array}$

$\mathrm{C}_{\mathrm{H}, 3}$

\begin{tabular}{lr}
\multicolumn{2}{c}{29} \\
Geometry: \\
C & 0.1416507718 \\
C & -0.0295054632 \\
C & 1.4212639610 \\
X & 0.0644224485 \\
Pd & -1.4451613407 \\
C & 2.5280000669 \\
C & 2.3420120098 \\
C & 1.0664898328 \\
P & -2.0557909729 \\
P & -1.5713185783 \\
H & -1.4696374119 \\
H & -3.3942573738 \\
H & -2.7441015239 \\
H & -0.6797865932 \\
H & 3.1986336455 \\
H & 0.9270578643 \\
H & -1.0235571505 \\
C & -2.0139743852 \\
C & -1.6783783060 \\
C & -2.6011766820 \\
H & -2.0048592673 \\
H & -0.6533727093 \\
H & -3.5468100201 \\
H & -2.8716828310 \\
&
\end{tabular}

36 Total energy:

1.4153297269

$-0.1527569958$

$-0.2352756619$

$-0.1145119609$

2.5366966092

1. 9199158499

$-2.4701031266$

2. 9692584905

2.1595848244

$-1.1016036061$

0.0130804232

1. 7485688342

1.1652715468

2.8847837363

3. 4010114380

0.2291517635

0.2816393446

$-0.0038059002$

$-0.3477007808$

$-0.3989887208$

0.4303307102

0.5379921902

0.0344226130

$-0.5822820107$

$-0.6871828864$

$-1.0342274936$

$-1.0369174483$

$-2.4394236033$

$-0.8620745544$

2. 6060989243

0.9467314707

0.3551522155

0.9572191534

$-1.6340412636$

$-1.5282325264$

$-0.5489028448$

$-1.7840716365$

$-2.4212867653$

$-1.8114133016$

$-0.5916133020$

$-0.0236555565$

$-2.2295087670$

$-3.3678885812$

$-2.2929207480$

$-0.1152356570$

$-1582.36939022067$

$-0.6536749561$

0.2435560644

0.1026798130

$-0.0650502252$

$-0.4144325088$

$-0.6711469318$

0.9182549589

$-0.1412793906$

$-2.0472735152$

$-0.3023376251$

1. 5010431329

$-0.3602751639$

$-1.7211478762$

0.6247427578

$-1.0552928553$

1.0507795228

0.9505347172

$-0.2634397120$

$-1.3769654714$

$-1.2807642234$

2. 0085508712

1. 8267058409

$-0.3383449002$

$-2.3237915954$

$-2.1528506917$

20 Total energy:

0.0597377345

$-0.1350748021$

$-0.0636213946$

$-0.0410415542$

5.0270798234

$-0.3541449977$

$-0.5366213638$

$-0.4280300190$

6.2152452972

6.2619273904

6.4100394307

6.1655148927

6.2300936557

6.4869238682

$-0.7670984056$

$-0.5758365998$

$-0.0482250809$

8.0049905533

8.0365397568

8.2790266292

8.6759866285

8.3421819925

7. 7336919050

9.3435794619
$-1621.65798977584$

0.0131865693

1. 3870980241

$-0.5310385968$

0.7124034597

0.2685484327

1. 6418303886

2. 2040952071

$-1.3574274296$

2.4579481493

$-2.6431950242$

$-1.8376867939$

3. 2631702416

3. 5491147964

2. 2699548813

3. 2714157575

1. 8176248857

$-0.7816718321$

1. 8443867892

0.6247537223

2.6733819734

1.5971891100

0.7518262994

0.6453446578
0.5179643387 


$\begin{array}{lr}\mathrm{H} & -0.9614432954 \\ \mathrm{H} & -2.5427759836 \\ \mathrm{H} & -0.7032141419 \\ \mathrm{Cl} & 1.6467026769 \\ \mathrm{H} & 3.5135602422\end{array}$

8.3157281425

8.6230689469

0.2882086902

0.1550662806

$-0.4433116976$

\section{$I_{\mathrm{H}, 3}{ }^{12}$}

\begin{tabular}{|c|c|}
\hline \multicolumn{2}{|c|}{ Geometry: } \\
\hline $\mathrm{Pd}$ & 0.0159686353 \\
\hline $\mathrm{C}$ & 0.0066026227 \\
\hline $\mathrm{Cl}$ & 1.7368148099 \\
\hline $\mathrm{C}$ & -0.5822210627 \\
\hline C & -0.8067146158 \\
\hline $\mathrm{C}$ & -2.1850074502 \\
\hline $\mathrm{C}$ & -2.7915372201 \\
\hline $\mathrm{C}$ & -2.0038463132 \\
\hline $\mathrm{P}$ & 0.8054778187 \\
\hline $\mathrm{P}$ & -0.3034698219 \\
\hline $\mathrm{H}$ & 0.5491555049 \\
\hline $\mathrm{H}$ & 2.2089437338 \\
\hline $\mathrm{H}$ & 0.7749399548 \\
\hline $\mathrm{H}$ & -1.3168287159 \\
\hline $\mathrm{H}$ & -0.3330787882 \\
\hline $\mathrm{H}$ & -2.8025140330 \\
\hline $\mathrm{H}$ & -3.8725127399 \\
\hline $\mathrm{H}$ & -2.4612454909 \\
\hline $\mathrm{H}$ & 0.0308632708 \\
\hline C & 0.3014761676 \\
\hline $\mathrm{C}$ & -0.5093969104 \\
\hline $\mathrm{C}$ & 0.5253265523 \\
\hline $\mathrm{H}$ & -0.4727266611 \\
\hline $\mathrm{H}$ & -1.5205783185 \\
\hline $\mathrm{H}$ & 1.5426267546 \\
\hline $\mathrm{H}$ & 0.4873343680 \\
\hline $\mathrm{H}$ & -0.7654887638 \\
\hline $\mathrm{H}$ & 0.8495683306 \\
\hline
\end{tabular}

\section{$I_{H, 3}{ }^{23}$}

$\begin{array}{lr}\text { Geometry: } \\ \text { Pd } & 0.0022212059 \\ \text { C } & 0.0034710871 \\ \text { C } & 1.3631022829 \\ \text { C } & -0.5424330262 \\ \text { C } & 0.1978977894 \\ \text { C } & 1.5497242683 \\ \text { C } & 2.1244796565 \\ \text { P } & -2.1904060238 \\ \text { P } & 1.2223183820 \\ \text { H } & -3.3583501920 \\ \text { H } & -2.8155272292 \\ \text { H } & 1.6397864809 \\ \text { H } & 2.4322927544 \\ \text { H } & 2.1364725237 \\ \text { H } & 3.1690742421 \\ \text { H } & 1.8672497932 \\ \text { C } & -2.2870584638 \\ \text { C } & 0.2027962097 \\ \text { C } & -1.2084976503 \\ \text { H } & 0.7563078029 \\ \text { H } & 0.1194241060 \\ \text { H } & -1.1511464494 \\ \text { H } & -1.5570601751 \\ \text { H } & -2.2059423448 \\ \text { H } & -3.2802455092 \\ \text { H } & -0.5325163993 \\ \text { Cl } & -2.2171220344 \\ \text { H } & -0.2508172554 \\ \text { X } & 0.6767856508\end{array}$

\section{$I_{H, 3}{ }^{34}$}

$$
\text { Geometry: }
$$

Pd $\quad-0.0006001800$

C $\quad-0.0042964633$

C $\quad 1.3487341370$
5 Total energy: 0.0206503042 $-0.0059228631$

$-0.0227866844$

$-1.2111369114$

1.0361164646

0.8967891401

$-0.2905052213$

$-1.3259742092$

2. 0595828521

$-1.1595960176$

3. 3869345812

2. 2374928512

$-1.9440228634$

$-2.1093091959$

1. 9327316388

1.7049360529

$-0.3912244734$

$-2.2550281198$

$-2.0937716101$

2. 3229458162

$-0.0459619100$

1.0978959795

$-0.6595684832$

0.3850160832

0.7034625041

1.4631016356

2.5936337683

3. 1886453043

2 Total energy: $-0.0031625492$ $-0.0021903839$ $-0.0106170434$ $-1.2021656411$ $-2.3639790035$ $-2.3567290475$ $-1.2033084009$ 0.0546414412 0.0031328197 $-0.5963175579$

1. 3198467897 1. 2491338785 $-0.6825205853$ $-3.2668366410$ $-1.1972607120$ $-0.5500563046$ $-0.5977517785$ 0.0213420727 $-0.4041389678$ $-1.6881309893$ 1.1142916986 $-0.1516125927$ $-1.6444795134$ $-0.3187774214$ 0.9308622770 $-1.1822861617$ $-3.2646027797$ $-0.0063732309$

2 Total energy: 0.0019271894 $-0.0032738842$ 0.0015053674 0.9304522595
$-0.8083487405$

$-1.5171887351$

$-0.6282255419$

$-2.2636219914$

$-0.1772092236$

$-1621.66157049427$

0.0230268869

2.3739910970

2. 8020837415

1. 9012365508

2.8966072854

2. 9031325409

2. 4287575541

1. 9454224146

$-0.8964944828$

$-1.9926236592$

$-0.4421674687$

$-1.0448927870$

$-2.4878614458$

$-2.3243989440$

3. 2834622708

3.2884487421

2. 4606164466

1.6086318839

1.7408841458

$-2.6885287731$

$-3.4898813927$

$-3.6053619218$

$-4.3990784363$

$-3.4380734654$

$-3.4722223562$

$-4.6401390784$

$-2.6870292090$

$-3.0861879668$

$-1621.66746610743$

$-0.0031898007$

2.3619427214

1. 9478995375

2. 8854183813

2. 9871903386

2. 5690042660

2.0631044240

$-0.8594103874$

$-2.0140709545$

$-0.3686001381$

$-1.0407313090$

$-2.5608833489$

$-2.3327174749$

2. 6595440360

1.7634337572

1. 7476809904

$-2.6332209098$

$-3.4703808394$

$-3.5798797457$

$-4.3964104386$

$-3.3736546920$

$-3.4709591783$

$-4.6074865047$

$-2.6024209721$

$-3.0350395296$

2. 4999846574

3. 4347491743

3. 3931289180

2.1332119275

$-1621.66631761752$

0.0051822692

2.3979287039

1. 9724087925 


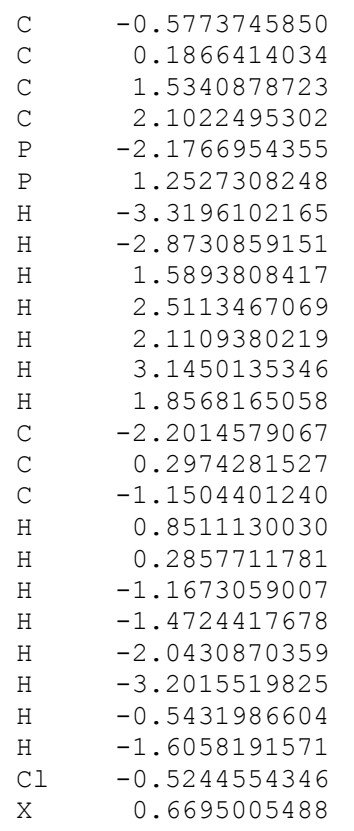

$-1.1984399828$ $-2.3477229872$ $-2.3642377352$ $-1.1974131222$ $-0.0619415519$ 0.0631341069 $-0.7669441281$ 1.1588368524 1.3313766634 $-0.5376266359$ $-3.2808704552$ $-1.1949160345$ 0.9405588374 $-0.7200251027$ $-0.6187709511$ $-0.0973990386$ $-0.3972370100$ $-1.7114834779$ 0.9990352989 $-0.3153299460$ $-1.8046337486$ $-0.5708064270$ 0.9314590521 $-1.2037048409$ $-3.8386728474$ $-0.0008604182$
2.9060908940

2.9634066238

2.5431970093

2.0599489520

$-0.8919146956$

$-1.9903204605$

$-0.4107521073$

$-1.1242726210$

$-2.5423493318$

$-2.2922243584$

2.6105112937

1.7528893389

1.7742816748

$-2.6498146181$

$-3.4564180508$

$-3.5952008827$

$-4.3762742504$

$-3.3499367667$

$-3.5100655135$

$-4.6230532971$

$-2.5877284565$

$-3.0726455979$

2.5232008803

3.2528812715

3.5839868287

2.1705973695

\section{$\mathbf{T}_{\mathrm{SH}, 3}{ }^{\text {ox }}$
28}

Geometry: 16 Total energy: -1621.63833789074

Pd 0.0024066842

C $\quad 0.0088580896$

$\mathrm{Cl} \quad 1.9937102248$

$\begin{array}{lr}\mathrm{C} 1 & 1.9937102248 \\ \mathrm{C} & -0.3939048363\end{array}$

C $\quad-0.4723977727$

$-1.4634711090$

$-1.9217227831$

$-1.3717926918$

$-1.9564529398$

0.2650654685

$-2.2752862414$

$-3.2106289555$

$-0.3175239748$

1.4950737390

$-0.0757522534$

$-1.8568297158$

$-2.6763639569$

$-1.7097684524$

0.0376917078

$-2.2382676704$

$-0.5732268051$

$-2.0361363886$

$-0.5338864515$

0.0292374427

$-2.6010104167$

$-2.4970315799$

$-1.5434925196$

$-3.2507293239$

$-0.0005030496$

0.0000738659

0.0015484694

$-1.1300294342$

1.2783265804

1.3998432275

0.2770639513

$-0.9833993624$

$-1.2770208814$

0.8651496499

$-2.5368570449$

$-0.6726420867$

2.1262380943

1.1119349068

2.1575158748

2.3861742335

0.3794940617

$-1.8616385536$

$-0.0049681260$

2.1605264137

1.5595858567

2. 8964870261

2.4911812243

3.4740246125

4.1669372559

3.8772410105

$-0.4866601617$

$-2.2570458305$

0.0950437867

$-0.2036298467$

$-2.5609013886$

$-2.9276766972$

1.9931387488

3.7095195264

4.9416696362

4.4230657162

2.6797085703

$-2.1018206980$

$-1.7109905050$

$-0.1574376462$

$-0.5455098620$

0.3908061904

$-2.2866749516$

$-3.5854642784$

$-3.2788472433$

$-4.5331678177$

$-3.7188039344$

0.0653086372

0.3375047183

$-0.8469316645$

$-2.9463887262$

$-4.2293413022$

$-2.5314320644$

$-2.5248499566$

$-2.1142814402$

$-2.4023793212$

\section{$\mathrm{II}_{3}$}

$$
28
$$

$\begin{array}{lr}\text { Geometry: } \\ \text { Pd } & 0.0654091193 \\ \text { C } & -0.0120505487 \\ \text { Cl } & 2.4631510233 \\ \text { C } & 0.1349959105 \\ \text { C } & -0.1604791882 \\ \text { C } & -0.1556715332 \\ \text { C } & -0.0112682312 \\ \text { C } & 0.1353907445 \\ \text { P } & -2.2146004723 \\ \text { P } & 0.2251065343 \\ \text { H } & -2.9130505980 \\ \text { H } & -2.7426540936 \\ \text { H } & 0.7382711643 \\ \text { H } & 1.1020596025 \\ \text { H } & -0.2590292652\end{array}$

76 Total energy: $-0.0168562447$ 0.0022429939

$-0.1312517515$

$-1.1942546791$

1.2043561809

1.2114520017

0.0158824853

$-1.1864119076$

0.1060039647

$-0.0052408742$

$-0.7130833117$

1.3582312017

1.1856217748

$-0.9222359721$

2.1470399142

$-1621.68741790966$

$-0.0205110176$

2.0281986724

0.0314086605

2.7413231761

2.7300660436

4.1324511710

4.8414666833

4.1419205125

0.0294132977

$-2.4348749780$

0.9420056631

0.4231096760

$-2.9996377815$

$-3.0523265877$

2.1960673186 


$\begin{array}{rrr}-0.2593123802 & 2.1542537491 & 4.6654576812 \\ -0.0060866492 & 0.0205607666 & 5.9285670661 \\ 0.2607459870 & -2.1213612201 & 4.6842943096 \\ 0.2754672575 & -2.1347811742 & 2.2149826635 \\ -3.1518444093 & -0.2229454040 & -1.5474420128 \\ -1.3383427426 & -0.2458008310 & -3.4292551834 \\ -2.5809594699 & 0.4414835791 & -2.8207591102 \\ -1.1836122255 & 0.1126149859 & -4.4531141238 \\ -1.5166876093 & -1.3270913254 & -3.4973573331 \\ -2.3762605427 & 1.5058370486 & -2.6361486019 \\ -3.3733002143 & 0.4205049964 & -3.5803086844 \\ -3.1811881712 & -1.3122221933 & -1.6809681737 \\ -4.1875254869 & 0.1025450059 & -1.3948809875\end{array}$

$\mathrm{II}_{\mathrm{SH}, 3}$

Geometry: 28 Total energy: $\quad-2020.55066944179$

0.0464817582

Cl 2.5209291826

$\begin{array}{lr}C 1 & 2.5209291826 \\ C & -0.4737912997\end{array}$

0.5709184729

0.5719341287

0.0515548050

$-0.4674291356$

$-2.1936775094$

0.0671538555

$-2.9278378804$

$-2.5692334958$

0.8973552117

0.5782221275

1.0043951561

0.9879703079

0.0538785117

$-0.8711828937$

$-0.8838993388$

$-3.2408173775$

$-1.5220693336$

$-2.5277431524$

$-1.2836310720$

$-1.9615728332$

$-2.0402167865$

$-3.3056217246$

$-3.6236349279$

$-4.0924269828$

$-1.9144338625$

$-0.0507482450$

0.0266465683

0.0676644577

2.0774680173

$-0.1182356261 \quad-0.0211866385$

$\begin{array}{ll}-0.9883242619 & 2.8424143523\end{array}$

$1.1809591208 \quad 2.7516140411$

$1.2403076219 \quad 4.1515237859$

$0.1811798158 \quad 4.9049068957$

$\begin{array}{lll}-0.9363954787 & 4.2434676001\end{array}$

$\begin{array}{ll}0.2023173247 & 0.1247073887\end{array}$

$-0.2547503896-2.3752966022$

$-0.6869055335 \quad 0.9263366890$

$\begin{array}{ll}1.4219643676 & 0.7645577276\end{array}$

$0.6647210503 \quad-3.0748119784$

$-1.4465564329-2.9219369770$

$2.0000729503 \quad 2.1833207218$

$2.1129476813 \quad 4.6536509139$

$0.2257003478 \quad 5.9924640427$

$-1.7713166530 \quad 4.8146834875$

$\begin{array}{ll}-1.8653479670 & 2.3453405160\end{array}$

$0.3443881051-1.4109310597$

$-0.0428855552-3.3296128050$

$0.9123144363-2.6544687886$

$0.3257877252-4.3354611371$

$-1.0408140856-3.4242291668$

$1.8677305080 \quad-2.4076641341$

$1.1554552530-3.3935415660$

$-0.6583032887-1.6223510073$

$0.9889063466-1.1564807869$

$-2.8214762546-1.1759038509$

$\begin{array}{lll}-0.9123165371 & -3.2974046409 & -0.4010852361\end{array}$

\section{$\mathrm{TS}_{\mathrm{SH}, 3}^{\mathrm{SH}-\mathrm{Cl}}$}

30

Geometry: $\quad 33$ Total energy: $\quad-2020.53879812158$

Pd $\quad 0.0572524762$

C $\quad 0.1419179570$

$\mathrm{Cl} \quad 2.5712850510$

$-0.8444702760$

1.1961083465

1.2609907240

0.2714586280

$-0.7766325939$

$-1.1702303993$

0.0434686805

$-2.0268507878$

$-0.5561319726$

1.0391276375

0.1999680740

1. 9827932099

2.0888651199

0.3190208482

$-1.5470066108$

$-1.6551079262$

$-2.3886143322$

$-1.4719727425$

$-1.8048661099$

$-1.3424398063$

$-2.3017826346$

$-0.9176729167$

$-0.0576454490$

0.0470262409

$-0.0063693386$

$-0.6399685401$

$-0.4976195015$

0.7663957843

0351434352

$-0.3352241095$

2. 8704246308

2.6215544688

$0.9439385154 \quad 4.0098573569$

$0.4018656183 \quad 4.8380785623$

$-0.3246798480 \quad 4.2615019122$

$\begin{array}{ll}1.9252163451 & 0.0490800717\end{array}$

$-0.0942029426 \quad-2.3814734804$

$2.1615527574 \quad 1.1533019793$

3.2140661671 0.0609997289

$0.6597775206-3.0666686323$

$-1.3174230708 \quad-3.0619238476$

$1.1715169868 \quad 1.9902788847$

$1.5052923488 \quad 4.4428457359$

$0.5418770110 \quad 5.9169600125$

$-0.7622652538 \quad 4.8959305051$

$-1.0738004144 \quad 2.4310944975$

$2.2037079214-1.3427770399$

$0.6099281014-3.2082180399$

$2.0528569718 \quad-2.7649790313$

$0.5897739587 \quad-4.2975458046$

$-0.0628113757 \quad-2.9606866319$

$2.6955229756-2.8740056698$

$2.4553099787 \quad-3.4672847729$ 


\section{$-3.1933067874$ \\ $-2.8332961215$ \\ $-1.6615930848$ \\ $-0.6440592277$}

II ' $\mathrm{Cl}, 3$
1.4714844226

3. 2010408481

$-2.2072107821$

$-3.0969104173$
$-1.1998495876$

$-1.2353699164$

$-0.4452113287$

$-0.3795959013$
Geometry:

Pd $\quad 0.1474311644$

0.1532731638

2. 5280646742

0.0467155184

0.2696241034

0.2846720547

0.1793760866

0.0595802350

$-2.1898696987$

0.0419735511

$-2.8695003374$

$-2.8043050701$

$-0.3520460179$

1.2055966105

0.3634421310

0.3835798100

0.1932477261

$-0.0186508772$

$-0.0314068521$

$-2.9727163266$

$-1.1694536926$

$-2.6367289686$

$-1.1139678168$

$-0.8158061209$

$-2.9636889537$

$-3.2429665298$

$-2.6775167440$

$-4.0602653699$

2.7442605019

$-2.9989576276$

\section{II' 3}

Geometry:

$\begin{array}{lr}\text { Pd } & 0.0287812795 \\ \text { C } & 0.0746146244 \\ \text { S } & 2.3924997463 \\ \text { C } & 0.0248054125 \\ \text { C } & 0.1458608905 \\ \text { C } & 0.1703313165 \\ \text { C } & 0.1231217247 \\ \mathrm{C} & 0.0502040737 \\ \mathrm{P} & -2.3023236730 \\ \mathrm{P} & -0.0086565245 \\ \mathrm{H} & -2.9448684502 \\ \mathrm{H} & -2.8814366410 \\ \mathrm{H} & 0.0941990505 \\ \mathrm{H} & 1.0047427970 \\ \mathrm{H} & 0.2061215471 \\ \mathrm{H} & 0.2361801579 \\ \mathrm{H} & 0.1499198210 \\ \mathrm{H} & 0.0231870383 \\ \mathrm{H} & -0.0093062638 \\ \mathrm{C} & -3.2972606393 \\ \mathrm{C} & -1.5383931541 \\ \mathrm{C} & -2.8643749917 \\ \mathrm{H} & -1.4712428114 \\ \mathrm{H} & -1.5280838751 \\ \mathrm{H} & -2.8339786855 \\ \mathrm{H} & -3.6566617382 \\ \mathrm{H} & -3.2180733373 \\ \mathrm{H} & -4.3521980586 \\ \mathrm{H} & 2.6241886607\end{array}$

29 Total energy: 0.0784538457

$-0.0464748006$

$-0.1126925314$

$-1.2945599479$

1. 1114272732

1.0231450782

$-0.2242955911$

$-1.3834635972$

0.2004840174

0.1900853762

$-0.4610821203$

1. 4809687775

1. 4656621793

$-0.4590848058$

2. 0897823331

1. 9320205805

$-0.2931721207$

$-2.3610032548$

$-2.2081928478$

$-0.4270845555$

$-0.4560587161$

0.1070737061

$-0.2387076897$

$-1.5487866811$

1.2060559295

$-0.1580593194$

$-1.5219589873$

$-0.1835189573$

$-0.1403311113$
$-2020.57148537539$

0.1818794657

2. 2139572086

0.1687717683

2. 7890843816

3.0804699287

4. 4714795825

5.0294000316

4.1798777093

0.1542665060

$-2.2086988723$

1. 3124862517

$-0.0978991159$

$-2.7075167904$

$-2.9833158561$

2. 6682326930

5.1183323689

6.1092799733

4. 5976381057

2.1473599463

$-1.1452439595$

$-3.0562946668$

$-2.6108850263$

$-4.1379480193$

$-2.8686543348$

$-2.8127473862$

$-3.2437836626$

$-0.9101233666$

$-1.0196310137$

1. 4807186635

$-2.1600522272$

$-1560.26471569604$

$-0.0144606975$

2.0369143957

$-0.0063028108$

2. 6762296866

2. 8253488951

4.2247289721

4.8539667305

4.0746514699

0.1038205328

$-2.4309622035$

1. 1712726270

0.2880321065

$-3.0402200616$

$-3.1724827389$

2. 3545780913

4.8193802382

5.9386927157

4.5520197992

2.0874268410

$-1.3437611605$

$-3.2818074963$

$-2.7258415994$

$-4.3536945708$

$-3.1774829457$

$-2.7147256506$

$-3.4381840301$

$-1.3304038436$

$-1.1721078844$

1. 3228869323

\section{$\mathrm{TS}_{\mathrm{SH}, 3}{ }^{\mathrm{RED}}$}

$\begin{array}{ll} & 29 \\ \text { Geometry: } & \\ \text { C } & -0.0338973803 \\ \text { S } & -0.0231406390\end{array}$

62 Total energy:

0.0573692026

$-1560.22889138569$

0.0586472767

2. 2111886741 


$\begin{array}{lr}\mathrm{Pd} & 1.9517788646 \\ \mathrm{C} & -0.3501433008 \\ \mathrm{C} & -0.4686962432 \\ \mathrm{C} & -1.1537078429 \\ \mathrm{C} & -1.4292984658 \\ \mathrm{C} & -1.0215546549 \\ \mathrm{P} & 3.2657467758 \\ \mathrm{P} & 3.9065371216 \\ \mathrm{H} & 2.7321077798 \\ \mathrm{H} & 3.7997099391 \\ \mathrm{H} & 4.6112855704 \\ \mathrm{H} & 3.8855581597 \\ \mathrm{H} & -0.2487828154 \\ \mathrm{H} & -1.4628871379 \\ \mathrm{H} & -1.9624304478 \\ \mathrm{H} & -1.2402002593 \\ \mathrm{H} & -0.0841849361 \\ \mathrm{C} & 4.8451616055 \\ \mathrm{C} & 5.3381219526 \\ \mathrm{C} & 5.7699568941 \\ \mathrm{H} & 6.1982291272 \\ \mathrm{H} & 5.0318387168 \\ \mathrm{H} & 5.9312042246 \\ \mathrm{H} & 6.7559279968 \\ \mathrm{H} & 4.5515652756 \\ \mathrm{H} & 5.3972195284 \\ \mathrm{H} & -0.4281100445 \\ \mathrm{H} & \end{array}$

0.0342204293

$-1.2547324885$

1. 1362945292

0.9017172701

$-0.4057265186$

$-1.4774271288$

0.5290209603

$-0.7470180708$

0.4045865123

1. 8363914787

0.2026590066

$-1.7635282660$

2. 1587812472

1. 7482295277

$-0.5814008291$

$-2.4988326746$

$-2.1006270966$

$-0.4528424340$

$-1.3886042701$

$-0.4701385691$

$-1.5696764711$

$-2.3649733159$

0.5540923641

$-0.8186686791$

$-1.4797754142$

$-0.0595486871$

1.6035636558
0.8529016418

$-0.3542441368$

$-0.7426207343$

$-1.9344217413$

$-2.3609494699$

$-1.5610429894$

$-1.1052613013$

2.1014075625

$-2.4144225942$

$-1.2838345858$

2. 8894530076

3.0964349944

$-0.4449071265$

$-2.5444852029$

$-3.2913178032$

$-1.8659581235$

0.2737267741

$-1.3273369086$

1.0740309647

$-0.0906938578$

1.7284855719

0.6757402411

0.2758750757

$-0.4271944125$

$-1.5814703238$

$-2.1884709810$

1. 9445492687

$\mathrm{C}_{\mathrm{H}, 4}$

32

Geometry: $\quad 70$ Total energy: $\quad-1660.97695755312$

$\begin{array}{lrrr}\mathrm{C} & -0.2962865212 & -0.0405775553 & 0.1324588193 \\ \mathrm{C} & -0.2206853307 & -0.0582478540 & 1.5286886881 \\ \mathrm{C} & 1.0284205082 & 0.0676890108 & 2.1383391711 \\ \mathrm{C} & 2.1954763523 & 0.2084857391 & 1.3845846934 \\ \mathrm{C} & 2.1055133338 & 0.2237169585 & -0.0113574676 \\ \mathrm{C} & 0.8619155434 & 0.0997906140 & -0.6390767919 \\ \mathrm{C} 1 & 1.1321051238 & 0.0467350742 & 3.8976161196 \\ \mathrm{H} & 3.1562344295 & 0.3040004971 & 1.8800093707 \\ \mathrm{H} & 3.0129204876 & 0.3286488010 & -0.6055255586 \\ \mathrm{H} & 0.7972023558 & 0.1125359946 & -1.7234411413 \\ \mathrm{H} & -1.2658899906 & -0.1374060721 & -0.3493937342 \\ \mathrm{H} & -1.1146141019 & -0.1671899736 & 2.1346326035 \\ \mathrm{X} & -0.2584423184 & -0.0494228971 & 0.8313791126 \\ \mathrm{Pd} & 5.0815696696 & 1.0816295640 & -2.4659334599 \\ \mathrm{P} & 6.0034019776 & -0.9545331092 & -3.0518464713 \\ \mathrm{P} & 5.8263269067 & 3.2555945228 & -2.7471730937 \\ \mathrm{C} & 7.4787108609 & 3.2114868411 & -3.6563783908 \\ \mathrm{C} & 8.2106770781 & 1.8621018040 & -3.4724734426 \\ \mathrm{C} & 7.7826734831 & 0.7587737924 & -4.4755398853 \\ \mathrm{C} & 7.6805287737 & -0.6551765564 & -3.8616084659 \\ \mathrm{H} & 6.3353604403 & -2.0779399538 & -2.2441701948 \\ \mathrm{H} & 5.3905630444 & -1.7164308414 & -4.0844522610 \\ \mathrm{H} & 5.2152485064 & 4.3530302209 & -3.4155914932 \\ \mathrm{H} & 6.2235096557 & 4.0031822847 & -1.6053190713 \\ \mathrm{H} & 8.0497416359 & 1.5074065953 & -2.4463627575 \\ \mathrm{H} & 9.2921536528 & 2.0288065487 & -3.5690546991 \\ \mathrm{H} & 7.2800692530 & 3.3960160561 & -4.7194971314 \\ \mathrm{H} & 8.0969141076 & 4.0438253431 & -3.3013462676 \\ \mathrm{H} & 7.8465902961 & -1.4276346049 & -4.6213935404 \\ \mathrm{H} & 8.4512397161 & -0.7963059521 & -3.0937735606 \\ \mathrm{H} & 8.5032120980 & 0.7322169334 & -5.3040621671 \\ \mathrm{H} & 6.8158412416 & 1.0223683817 & -4.9233006332 \\ & & & \end{array}$

$I_{H, 4}{ }^{23}$

\begin{tabular}{ll}
\multicolumn{2}{l}{31} \\
Geometry: \\
C & 0.0856903669 \\
$\mathrm{Cl}$ & 0.1242922407 \\
$\mathrm{Pd}$ & 2.9110828409 \\
$\mathrm{C}$ & 5.6950238565 \\
$\mathrm{C}$ & 5.9555261121 \\
$\mathrm{C}$ & 6.4713158735 \\
$\mathrm{C}$ & 5.7561409296 \\
$\mathrm{P}$ & 4.1549032906 \\
$\mathrm{P}$ & 3.9897091391 \\
$\mathrm{H}$ & 3.6568130181
\end{tabular}

34 Total energy:

0.0078176397

0.1724391760

0.1013570271

$-1.2508037755$

$-2.0161367201$

$-1.1292885803$

$-1.3581965452$

$-0.3763527044$

$-0.4557356159$

$-0.9732237586$

$-1660.97686666579$

0.0511477676

1.8068976516

$-1.4456375398$

0.5669393010

$-0.7464908953$

$-1.9114986761$

$-3.2579407077$

$-3.3936683819$

0.5962967009

$-4.5887030186$ 


$\begin{array}{rr}\mathrm{H} & 4.6840590424 \\ \mathrm{H} & 4.1597480812 \\ \mathrm{H} & 3.3033832412 \\ \mathrm{H} & 5.0349846011 \\ \mathrm{H} & 6.6888208519 \\ \mathrm{H} & 6.4449641454 \\ \mathrm{H} & 5.7866824556 \\ \mathrm{H} & 6.4084316106 \\ \mathrm{H} & 5.5097463522 \\ \mathrm{H} & 7.5439160050 \\ \mathrm{H} & 6.3889152634 \\ \mathrm{C} & -0.6193785929 \\ \mathrm{C} & -0.6587366441 \\ \mathrm{C} & -0.0039245413 \\ \mathrm{C} & 0.7290542659 \\ \mathrm{C} & 0.7861710649 \\ \mathrm{H} & -1.1407497479 \\ \mathrm{H} & -1.2180143107 \\ \mathrm{H} & -0.0529751981 \\ \mathrm{H} & 1.0791833445 \\ \mathrm{H} & 1.1680629056\end{array}$

$I_{H, 4}{ }^{34}$
0.7739211724 0.3683303534 $-1.4861811529$ $-2.5318723779$ $-2.8100291150$ $-0.4613865635$ $-1.9190004112$ $-1.0895250092$ $-2.4205856097$ $-1.3159033067$ $-0.0701936600$ $-1.0335271305$ $-1.1405569724$ $-0.2145504529$ 0.8470575274 0.9631626681 $-1.7511863616$ $-1.9545215413$ $-0.2898525101$ 1. 6683152544 1. 8617805565

18 Total energy: 0.0506996223 $-0.0599194364$ $-0.0440339990$ $-1.2326400179$ $-2.4081366472$ $-2.4468659684$ $-1.2761589872$ 0.3187240194 0.0727347293 0.4840316728 $-0.6192766718$ $-0.1233476992$ $-0.1171351088$ 0.8989498299 $-1.2221771092$ $-0.2846849431$ 1. 6501418412 0.6723229333 $-1.3617451856$ $-1.4344861118$ $-1.0640484430$ 0.8980671975 $-0.7463756277$ 0.6441874906 $-0.9861164909$ 0.7747162906 1.3889527991 $-3.8953485739$ $-3.3809655930$ $-1.2929615310$ 0.8710135525 $-0.0520114836$
$-4.0418591190$

1. 7441809775

1.2808767110

$-1.0522682892$

$-0.5528459355$

0.6991295280

1. 4307541848

$-4.0966890657$

$-3.3790102914$

$-2.0512507126$

$-1.6342699503$

$-0.5226978091$

$-1.9319300810$

$-2.7272280346$

$-2.1383948987$

$-0.7252106687$

0.1018924275

$-2.3858704760$

$-3.8105951812$

$-2.7570191729$

$-0.2518900197$

$-1660.97686249888$

0.0348886720

2.5105321953

2. 1493595015

2. 2235952148

2. 6244861211

2. 9773798904

2. 9253145316

$-0.6126298916$

$-2.4265520112$

$-3.3580639862$

$-3.5785008039$

$-3.4646305015$

$-1.6777761354$

1.9633163084

1.9675059543

$-0.0466164092$

$-0.4802629360$

$-1.8559524189$

$-1.6945116224$

$-2.8603056524$

$-4.5715580559$

$-3.8556069816$

$-4.0629159833$

$-2.6544935325$

$-2.5733306006$

$-4.3314434820$

$-2.9590987075$

2. 7050739175

3.2944445282

3. 2140496408

2. 6210157491

2. 3307362890

\section{$\mathrm{T}_{\mathrm{SH}, 4}{ }^{\mathrm{ox}}$}

$\begin{array}{ll}\text { Geometry: } \\ \text { C } & 0.0309426688 \\ \text { Cl } & 0.0246014663 \\ \text { Pd } & 2.1157952466 \\ \text { C } & 5.6558210445 \\ \text { C } & 6.3402423052 \\ \text { C } & 5.8414119789 \\ \text { C } & 4.8149942571 \\ \text { P } & 3.0108803455 \\ \text { P } & 4.0955417181 \\ \text { H } & 2.4305020622 \\ \text { H } & 2.8861413584 \\ \text { H } & 4.0300392740 \\ \text { H } & 4.6788221909 \\ \text { H } & 6.3460191083 \\ \text { H } & 7.3952364669 \\ \text { H } & 5.3952647937\end{array}$

24 Total energy:

0.0074149205

0.1048041767

0.0102224240

1.2017138641

$-0.0979790344$

$-0.7520202182$

$-1.9050331651$

$-1.4014889852$

1. 0001717493

$-2.6833814131$

$-0.9736578211$

2. 3223794584

0.4288674476

$-0.8465615602$

0.1613821982

1. 8272985117

\footnotetext{
$-1660.94691237139$

$-0.0359893410$

2. 0224857264

0.5810213179

0.5480183293

0.0658004391

$-1.2483602818$

$-1.1437363957$

$-1.1111238919$

1. 5658469034

$-1.3201272107$

$-2.4600136057$

2.0834175857

2.7303230095

0.8710143711

$-0.0927091772$

$-0.3157552950$
} 


$\begin{array}{rrr}6.3727858225 & 1.7757973795 & 1.1461435922 \\ 4.9552226377 & -2.5853637910 & -1.9916993589 \\ 5.0041250099 & -2.4930364240 & -0.2363422138 \\ 6.7246141191 & -1.1754863387 & -1.7442927438 \\ 5.4732711981 & 0.0269742984 & -1.9316454160 \\ -0.5718753916 & -1.1425932261 & -0.5812768516 \\ -1.2302263324 & -1.0453080890 & -1.8050364277 \\ -1.3340129880 & 0.1861328156 & -2.4742644800 \\ -0.7870793640 & 1.3309950353 & -1.8900514237 \\ -0.1319025734 & 1.2602523533 & -0.6538297904 \\ -0.5021017329 & -2.0917111111 & -0.0594725725 \\ -1.6677148670 & -1.9399479048 & -2.2429150759 \\ -1.8581481380 & 0.2505177013 & -3.4236434632 \\ -0.8871504115 & 2.2964917664 & -2.3812184556 \\ 0.2422662663 & 2.1589563001 & -0.1738670708\end{array}$

$\mathrm{II}_{4}$

31

$\begin{array}{lrrrr}\text { Geometry: } & 43 \text { Total } & \text { energy: } & -1660.99765303191 \\ \mathrm{C} & -2.8999820439 & 1.8234565338 & -1.7712976170 \\ \mathrm{C} & -2.1961565622 & 3.1958821057 & -1.7875455032 \\ \mathrm{C} & -0.7010573164 & 3.2382226458 & -2.1840101822 \\ \mathrm{C} & 0.3458219973 & 3.1501795322 & -1.0463983031 \\ \mathrm{P} & 1.0066778436 & 1.4592555015 & -0.6393213171 \\ \mathrm{Pd} & -0.3254310620 & -0.2065716399 & 0.1785454923 \\ \mathrm{C} & 1.4568070029 & -1.1276093694 & 0.6099460589 \\ \mathrm{P} & -2.5173649701 & 0.7000076499 & -0.3336323608 \\ \mathrm{Cl} & -1.5157662118 & -2.0135494355 & 1.2245387051 \\ \mathrm{H} & 2.1008998918 & 1.8038452821 & 0.1818589353 \\ \mathrm{H} & 1.7229772267 & 1.1528746238 & -1.8213133268 \\ \mathrm{H} & -3.4747846256 & -0.3137759251 & -0.5386385267 \\ \mathrm{H} & -3.1728192392 & 1.3906050805 & 0.7158622804 \\ \mathrm{H} & -2.3371414981 & 3.7057099511 & -0.8234636888 \\ \mathrm{H} & -2.7383970501 & 3.8068353422 & -2.5212673003 \\ \mathrm{H} & -2.6456560292 & 1.2664720216 & -2.6828894691 \\ \mathrm{H} & -3.9852509321 & 1.9788871125 & -1.7933445963 \\ \mathrm{H} & 1.2161379090 & 3.7664458106 & -1.3036348691 \\ \mathrm{H} & -0.0635118010 & 3.5663664933 & -0.1170810036 \\ \mathrm{H} & -0.5321660587 & 4.2023263609 & -2.6794623208 \\ \mathrm{H} & -0.5020430261 & 2.4790110916 & -2.9541204624 \\ \mathrm{C} & 1.9600213479 & -1.1185080423 & 1.9163863599 \\ \mathrm{C} & 3.1506063030 & -1.7915014670 & 2.2206204563 \\ \mathrm{C} & 3.8491813621 & -2.4806576706 & 1.2229619343 \\ \mathrm{C} & 3.3474661965 & -2.4953053073 & -0.0808096128 \\ \mathrm{C} & 2.1578628652 & -1.8173055175 & -0.3869942846 \\ \mathrm{H} & 1.4160237423 & -0.6135997778 & 2.7099792578 \\ \mathrm{H} & 3.5253223790 & -1.7846428852 & 3.2419560548 \\ \mathrm{H} & 4.7715484643 & -3.0048489426 & 1.4617433157 \\ \mathrm{H} & 3.8754301712 & -3.0360826491 & -0.8637801710 \\ \mathrm{H} & 1.7785434353 & -1.8501698843 & -1.4059464516 \\ & & & \end{array}$

$\mathrm{C}_{\mathrm{H}, 5}$

$\begin{array}{lr}\text { Geometry: } \\ \text { Pd } & -0.0102781375 \\ \text { P } & -0.0003307407 \\ \text { P } & 1.5821381274 \\ \text { C } & 3.1845671450 \\ \text { C } & 3.2212299822 \\ \text { C } & 3.2940433640 \\ \text { C } & 2.8859707217 \\ \text { C } & 1.5329867720 \\ \text { H } & -0.0508624197 \\ \text { H } & -0.9543284298 \\ \text { H } & 1.5554802683 \\ \text { H } & 2.0717409470 \\ \text { H } & 4.0453572016 \\ \text { H } & 3.2461936843 \\ \text { H } & 4.0829974798 \\ \text { H } & 2.3212998337 \\ \text { H } & 1.5442173724 \\ \text { H } & 1.4012748850 \\ \text { H } & 4.3156697598 \\ \text { H } & 2.6398999968 \\ \text { H } & 3.6463713087 \\ \text { H } & 2.8802198689 \\ \text { Cl } & -3.0195192753 \\ & \end{array}$

75 Total energy: $-0.0076797211$ $-0.0015629419$ $-0.0057176811$ 0.8049894548 1.0215830473 $-0.2722171412$ $-0.0802827837$ $-0.7063345830$ 1. 2442216782 $-0.6451091541$ 0.5222257668 $-1.2807233579$ 0.2149780164 1. 7764508997 1.6589081489 1.5828004916 $-1.7777891525$ $-0.6239205099$ $-0.6739801380$ $-1.0307235796$ $-0.5297470237$ 0.9901995562 1. 4190689065

$-1700.29085462763$

$-0.0133385960$

2.3010879130

$-1.6919987164$

$-1.1062369359$

0.4152645116

1. 2480293568

2. 7313264236

3.1320994058

2. 9877436635

3.1364692032

$-3.0144543178$

$-2.0856981781$

$-1.4443318399$

$-1.6107971139$

0.6642004541

0.6977761244

2. 8927551705

4. 2180314253

1.1902566812

0.7987057609

3.3838201596

2. 9815329125

$-2.5751619548$ 
-3.4308777799
-4.3303887166
-4.6735778934
-4.1194809121
-3.2124412055
-2.8584709374
-4.7535812812
-5.3768552806
-4.3909712224
-2.7693090737
-2.1529156748

$I_{H, 5}{ }^{23}$

Geomet

Pd $\quad 0.0023971896$

C $\quad-0.0121313774$

1. 3425883005

$-0.5480943934$

2.1197453767

1.5563359047

0.2068881668

$-2.2982859754$

$-2.7659017224$

$-1.6053520582$

$-1.2247201050$

0.2143715284

1. 2770642723

1. 6073604552

$-0.5773368194$

0.6679592905

2.1766377872

2. 8605559137

$-3.4292381189$

$-2.7851877445$

$-3.0246128072$

$-3.6661235639$

$-1.8626130877$

$-0.7383534806$

$-1.9384838287$

$-1.3425501002$

0.2226560077

0.5296600746

0.9796175986

2.2275713816

1. 8204410229

3. 1650282718

2.1534642128

$-0.2344378136$

$\mathrm{Cl} \quad-2.2256667304$

\section{$I_{H, 5} 34$}

Geometry:

$\mathrm{Pd} \quad-0.0326541118$

$-0.0963950215$

1. 2660382857

$-0.6022723487$

2.1040068485

1. 6036165456

0.2446605056

$-2.2794531494$

$-2.5171351952$

$-1.3066921912$

$-1.1039992256$

0.3351950295

1. 2539811441

1. 6128815247

$-0.7206724334$

0.5873211705

2. 3482883052

2.7677120948

$-3.3821397036$

$-2.9563248220$

$-2.6448785579$

$-3.4439913882$
0.3668152317

0.8207040827

$-0.0249355419$

$-1.3058606757$

$-1.7390401362$

$-0.9050183260$

1.8170490590

0.3220367518

$-1.9601596392$

$-2.7288156646$

$-1.2400661349$

5 Total energy:

0.0204732155

0.0058249269

0.0009013828

1.1947211141

1. 1716540190

2. 3265677938

2. 3450015147

$-0.0283724160$

1. 1498482221

1. 3726926303

0.1282236185

0.1562410599

$-0.5852091954$

0.0389161539

$-0.9163254802$

0.0033532300

1.2989170568

$-0.6245679273$

0.1887278810

$-1.2552001817$

2.0998577312

0.7929960371

2.1938014842

1. 7122143341

0.0274020822

$-0.7777224302$

$-0.3134877808$

1.1946870754

$-1.6347364774$

$-0.5810739775$

$-0.9396325797$

1. 1538196922

3. 2253506087

3. 2425130413

1. 1943882272

14 Total energy:

$-0.0628801236$

0.0669567075

$-0.0567080632$

1.3112218108

1.0796000580

2. 3048373331

2. 4043531708

0.2098367356

1. 2597548854

1. 1813585733

$-0.2008647973$

$-0.4564422150$

$-1.2296226123$

$-0.4520461360$

$-0.8174244983$

0.0048974451

0.6813587020

$-1.2369752076$

0.7183024689

$-0.9750894146$

2. 2914312223

0.9773362946
$-1.2259623296$

$-0.2595285654$

0.7996355914

0.8902822336

$-0.0803475340$

$-1.1466719025$

$-0.3362059449$

1.5523765087

1. 7145401064

$-0.0127306095$

$-1.8991040881$

$-1700.29115475544$

$-0.0087470665$

2. 4484815121

2. 0446036817

2. 9977162306

2.1840394404

2. 7143998903

3.1297676988

$-0.5655295217$

$-1.9553168216$

$-2.9355196027$

$-3.7605719530$

$-4.3221524346$

$-3.4865217347$

$-1.7430626670$

2. 5196050972

2. 2457284645

$-2.0730015221$

$-1.5810111604$

0.2737586787

$-1.0975797835$

$-1.4731514715$

$-2.4707346008$

$-3.6192270405$

$-2.3558401147$

$-4.5892706011$

$-3.1518248689$

$-5.3135086522$

$-4.4833344215$

$-3.3765257124$

$-4.0327356488$

1. 7861319309

1.8895768933

2.8254947331

3. 5552862369

3. 5433231943

$-1700.29134127183$

0.0543884223

2. 5344821050

2.1803124516

2. 9788513547

2. 2659402548

2. 6872021193

3. 0426185305

$-0.6094542735$

$-2.1519430467$

$-3.0923131277$

$-3.7438950605$

$-4.2445034542$

$-3.2750564862$

$-1.5958785381$

2. 6211775650

2. 3567475139

$-2.0449843683$

$-1.3063045960$

0.1383304103

$-1.0068934539$

$-1.8014282048$

$-2.6660913637$ 


$$
\begin{array}{r}
-1.4105410708 \\
-0.4145807416 \\
-1.8078093470 \\
-1.3726379172 \\
0.2977553852 \\
0.8070047337 \\
0.8060834407 \\
2.2131758808 \\
1.6971929088 \\
3.1567871563 \\
2.2462843176
\end{array}
$$

$\mathrm{Cl} \quad-0.3839274425$

$\mathrm{H} \quad-1.6444309899$

\section{$\mathbf{T}_{\mathrm{SH}, 5}{ }^{\mathrm{ox}}$}

Geometry:

$\mathrm{Pd} \quad-0.0145376544$

P $\quad 0.0264649710$

P $\quad 2.2942887574$

2.2942887574
3.5005121306

3. 2212756868

3. 4784199109

2. 7050818328

1.3403371727

$-0.1996508196$

$-1.0879344945$

2. 5982435354

3. 0466757993

4. 5341732137

3. 3646221625

3.8395606579

2.1793332802

1. 4965420066

0.9092108872

4.5567205018

3. 2152641278

3. 3065363245

2. 5746591590

$-1.6317045061$

$-2.1173781947$

$-3.0777390741$

$-3.9735762785$

$-3.9545591546$

$-3.0363485438$

$-2.1343786048$

$-3.1059775360$

$-4.6977797091$

$-4.6653538586$

$-3.0296408301$

$-1.4611433160$
1. 9399653443

1. 4552988702

$-0.2981298831$

$-0.9948202106$

$-1.0476189934$

0.4983656674

$-2.2096679459$

$-1.4203393140$

$-1.0369274323$

0.9898027531

3. 1765715287

3.9605114429

1. 4055296892
$-3.8814059674$

$-2.5159645624$

$-4.5815796504$

$-3.0342046671$

$-5.1698805180$

$-4.5196970703$

$-3.0611200010$

$-3.7709155630$

2.0025768015

2. 0099716866

2.7549415740

3.5870789965

3. 2690595637
46 Total energy: $-0.0286380361$ 0.0590405220

$-0.0725049967$

1.1503980656

1.3723174633

0.1372936231

0.1564213160

$-0.5632846691$

1.3392391936

$-0.5749736331$

0.1565819093

$-1.2737302846$

0.8258003177

2.0932628023

2. 2058188232

1. 6991459101

$-1.6207866681$

$-0.5401438728$

0.0654589953

$-0.7826887262$

$-0.3373536501$

1.1946498834

0.7761003392

$-0.4761281757$

0.0481401111

$-0.8212939299$

$-2.1978674946$

$-2.6949617416$

$-1.8374087046$

1. 1146381466

$-0.4202261302$

$-2.8643330965$

$-3.7537295652$

$-2.2135890883$
$-1700.26179575036$

0.0092985722

2.4192736910

$-0.8431540526$

$-0.0769136609$

1. 4170448311

2. 3034162995

3. 6385463316

3. 6174672311

2. 9990350344

3.0249337744

$-2.2111323413$

$-0.7376097170$

$-0.2478142617$

$-0.6203102030$

1.7795527264

1.5209802914

3. 3652484917

4. 6255472998

2.5000264251

1. 7632685856

4. 4137915376

3.9769698330

$-1.7762479787$

$-0.1832567684$

0.7013501682

1.3198733857

1. 0392551329

0.1108699714

$-0.5324031213$

0.9026006300

2.0256873270

1. 5197377747

$-0.1389511842$

$-1.2966687134$

$\mathrm{II}_{5}$

34

Geometry: 55 Total energy: -1700.30877758812

Pd $\quad-0.0031855167$

0.0000983157

2. 3789030365

3.1078313040

2.7037079713

3.3204680313

2. 3680685620

1.4961548486

$-0.7283145602$

$-0.7344223884$

2.7267416936

3.3408732509

4.1979790244

2. 7007869395

2.9914045107

1.6084315218

2. 1151087804

1.1065625146

4. 1783174083

3.7390643661

2. 9759421606

1. 7369961186

$-0.0152480606$

0.0339474464

0.0847631417

2. 3395051083

$-0.0007598573$

1. 7010185214

2.3137460969

1. 6038104407

$-0.4902401930$

$-0.2762131930$

1.0775902406

2. 3104741920

3. 5029076414

. 3812188561

3. 4587888168

2.8630110736

1. 1774259806

2. 9434167012

$-0.9566638354$

$-0.3155539339$

$-0.8128351387$

1. 6758407921

2. 3045808772

3.3727721170

2. 3041401191

$-0.7599637026$

$-1.8183400831$

0.1669353785

$-0.3950201590$

$-1.0961516534$

1. 0839840935

1.1399574275

3.1885539881

4. 4655153461

2. 6595291305

2. 0230746556

.1920260304

$\begin{array}{ll}1.2936301479 & 4.4129303911 \\ 2.2689847967 & 3.6518386878\end{array}$ 


$\begin{array}{ll}\mathrm{C} & -2.7059285863 \\ \mathrm{C} & -4.1043010963 \\ \mathrm{C} & -4.8467121361 \\ \mathrm{C} & -4.1835413538 \\ \mathrm{C} & -2.7854260987 \\ \mathrm{H} & -2.1472496347 \\ \mathrm{H} & -4.6106170490 \\ \mathrm{H} & -5.9314102985 \\ \mathrm{H} & -4.7520041789 \\ \mathrm{H} & -2.2889706145 \\ \mathrm{C} & -2.0387396800 \\ \mathrm{Cl} & -0.3439175000\end{array}$

\section{$\mathrm{C}_{\mathrm{H}, 6}$}

Geometry:

Cl $\quad 0.0587794229$

C $\quad 0.0613342821$

$\mathrm{Pd} \quad 4.8707427326$

$\mathrm{P} \quad 5.0772615210$

P $\quad 4.6774680248$

C $\quad 5.4720965781$

C $\quad 3.8208977867$

4. 3025247332

3. 9145413257

5.0685076733

4. 6181331017

4.0454057797

5.8847574591

3. 9635925407

6.0267365241

6.5373586000

5.3376619106

4.6470373125

3. 5674180407

5.3908020351

3.8662872159

5.8153480922

5.5707790358

3.5040170538

3.1012760072

2. 7529879353

3. 9225397920

$-1.1645200312$

$-1.1728775934$

0.0302552215

1. 2457699406

1.2733914746

$-2.0916577678$

$-2.1219439242$

0.0200553527

2.1848766609

2. 2241426577

\section{$I_{H, 6}{ }^{23}$}

\begin{tabular}{lr}
\multicolumn{2}{c}{38} \\
Geometry: \\
Pd & 0.0111947389 \\
$\mathrm{C}$ & -0.0087028458 \\
$\mathrm{C}$ & 1.3479934951 \\
$\mathrm{C}$ & 1.5051628724 \\
$\mathrm{C}$ & 0.0377667173 \\
$\mathrm{C}$ & -0.4656906964 \\
$\mathrm{C}$ & -1.9927367063 \\
$\mathrm{C}$ & -2.8016558025 \\
$\mathrm{C}$ & -3.0722596244 \\
$\mathrm{P}$ & -2.3041849671 \\
$\mathrm{P}$ & 1.7521126769 \\
$\mathrm{H}$ & -3.0502581927 \\
$\mathrm{H}$ & -3.1188058991 \\
$\mathrm{H}$ & 3.1170920410 \\
$\mathrm{H}$ & 2.0500117663 \\
$\mathrm{H}$ & 2.1650203683 \\
$\mathrm{H}$ & 1.8297081371 \\
$\mathrm{H}$ & -0.0869340765 \\
$\mathrm{H}$ & -0.5690240908
\end{tabular}
1.2888630445
1. 3299896995
0.1461996405
$-1.0794296690$
$-1.1212442617$
2. 2218808745
2. 2898088851
0.1788794138
$-2.0066287724$
$-2.0818196551$
0.0637737778
0.0188023514
0.4100056996
0.5016579817
0.4808017948
0.3636164904
0.2699778029
0.4100357473
0.5816742664
0.5484995300
0.3345520050
0.1561679315
0.2914519351
$-2.3419608035$

2 Total energy:

0.1299283630

$-1739.60945194975$

$-0.1084564315$

0.0589224760

$-0.0424940600$

$-2.3439172025$

2. 0714313216

$-3.1035974249$

2. 2415188772

1. 3062295940

$-0.1863307303$

$-1.0951148654$

$-2.5038726138$

3. 1662107533

2.7621792731

$-3.1372844624$

$-3.0240586196$

$-2.9209844925$

$-4.1891066134$

$-3.1910270459$

$-2.4693200876$

1. 4019324452

1. 6986018032

$-1.1846576380$

$-0.5994654807$

$-0.5633326712$

$-0.2643100837$

2. 0721010124

3. 2865149046

0.0677389042

0.0111365103

$-0.0539243377$

$-0.0635978847$

$-0.0054024345$

0.1173648771

0.0180051774

$-0.0962894483$

$-0.1124231976$

$-0.0130332159$

1.6586413219

0.8692201141

0.9918941897

$-0.0874060839$

$-0.6746981840$

$-1.7487015347$

$-2.8782470383$

$-2.7183906297$

$-2.2595068807$

$-1.8135092847$

0.5596946380

$-0.3789897771$

1. 3764704544

1.8013418216

$-0.8673832787$

$-0.6089045494$

$-2.6697580919$

$-1.4940457205$

$-3.0013461387$

$-3.8067899479$

$-3.0622373488$

$-1.4174169910$

$-3.6654775649$

$-1.9846387447$

$-1.5598171528$

$-2.0666692802$

2. 3298893297

3. 7258801534

4. 4361222262

3. 7447897495

2. 3472151540

1. 7679593320

4.2559793498

5.5219740038

4. 2897210105

1. 8138715750

$-1739.60372473787$

$-0.0279519337$

2. 5472550628

2.1615979686

$-3.2676095632$

$-3.7223930396$

$-4.1194160920$

$-4.2909072363$

$-2.9900237333$

$-2.1246353823$

$-0.4113347630$

$-1.6007142549$

0.1803439326

0.1151440688

$-1.4101790370$

$-2.0259854402$

$-4.0211460151$

$-3.1359268374$

$-4.5768326589$

$-2.9058602376$ 


$$
\begin{array}{r}
-2.2942829738 \\
-3.7729430852 \\
0.0258718730 \\
-0.1425679142 \\
-2.1822048598 \\
-2.3889600850 \\
-2.6865844767 \\
-4.1508647044 \\
2.1362020793 \\
1.5766077109 \\
0.2250978331 \\
-0.5409524442 \\
1.8106173605 \\
3.1843109931 \\
2.1821354853 \\
-0.2133332848 \\
-2.2236761399 \\
-0.5851889574 \\
0.6508059398
\end{array}
$$

1.8765364557 1. 5363097776 1.0505502403

1. 5046512288 1. 7745737011 0.0347150614 $-1.0588217687$ $-0.3103651830$ 1.1119566369 2. 2750442606 2. 3065082779 1. 1589875864 $-0.9872047357$ 1.0879807341 3. 1696330799 3.2102612383 1. 1744327765 $-0.9479402622$ $-0.0373434927$
$-2.3947359772$

$-3.2617959772$

$-5.0603226126$

$-3.3749556825$

$-4.9283950416$

$-4.8430167607$

$-2.6139180943$

$-2.0080580434$

2. 3131764499

2. 8276555187

3. 2160804749

3.0805778559

1. 8810371112

2.0290148899

2. 9417925628

3. 6266124060

3. 6002809814

2. 5802319953

2. 3127193595

$I_{\mathrm{H}, 6}{ }^{34}$

38

Geometry: 84 Total energy: $\quad-1739.60318164425$

1.2437686624

1. 3553656541

$-0.1248640199$

$-0.6441736533$

$-2.1768477605$

$-2.9280203802$

$-3.1761589359$

$-2.3563911941$

1.6523476982

$-3.0926940825$

$-3.1618269600$

3.0305312694

1. 9138588041

1.9901846943

1. 6892901580

$-0.2714410698$

$-0.7076333359$

$-2.3886761138$

$-3.9057938801$

$-0.1898886064$

$-0.2923716824$

$-2.3934501977$

$-2.5948376759$

$-2.8059948224$

$-4.2525473871$

2.0856955989

1.6117934635

0.2805333959

$-0.5687739804$

1. 6531798026

3.1177254087

2.2561696026

$-0.3167320144$

$-1.5895087607$

$-0.7110509029$

0.5713343737

0.0017460729

$-0.0272979743$

0.0102086849

2. 5982885234

$-0.0914053504$

$-1.0365006309$

$-1.0675324857$

2.1506709983

$0.2695825794 \quad-4.2069195913$

$0.3885321735-4.3311577233$

$0.7531007865-3.0310586037$

$-0.3918213048 \quad-2.0117351300$

$-0.1243302065-0.3431310309$

$-0.0080878335-1.6919421327$

$\begin{array}{ll}-1.1089960062 & 0.3829741529\end{array}$

$\begin{array}{ll}0.9691573013 & 0.0860125152\end{array}$

$-0.3327481174-1.5205675600$

$1.2570088686-2.2868960396$

$-0.6759498796-4.0605484573$

$-2.0533451321 \quad-2.9991059587$

$-1.8467268353-4.4170390652$

$-1.3696490541-2.7785496882$

$1.5824473087 \quad-2.5517698963$

$1.1642102603-3.3137329613$

$0.4307572872-5.1943741961$

$1.0960226445-3.5766110388$

$1.1809783270 \quad-5.0606930780$

$-0.5352768551 \quad-4.7596648295$

$-1.3476165696 \quad-2.3993497857$

$-0.5236905322-1.8494863469$

$1.0366893890 \quad 2.2252534029$

$2.2461181959 \quad 2.7203716191$

$2.3259375927 \quad 3.1594239373$

$1.2303447993 \quad 3.1153837400$

$-1.0561612738 \quad 1.8673215141$

$0.9616314054 \quad 1.8924271068$

$3.1170728355 \quad 2.7733166618$

3.8575157333 3.7951383800

$1.3122923385 \quad 3.4747644153$

$-0.8775444354 \quad 2.6600540139$

$\begin{array}{ll}-0.0401741826 & 2.3740997777\end{array}$

$\mathrm{T}_{\mathrm{SH}, 6}{ }_{37}$

eometry: 4 Total energy: -1739.57264833204

C 0.0017677430

$\mathrm{Pd} \quad 2.0921077628$

P $\quad 2.9876011556$

3.7272152491

4.4679061904

5.5789247032

6.4565743365

7. 1412934541

6.2282244989

5.5308775506

3. 3230715777

0.0148142198

1. 4550229027

$1.2466086927 \quad 3.2754453076$

$-1.1481307230 \quad 0.0027301922$

$0.6350385098 \quad 4.2517592460$

$-1.4557335682 \quad 0.1276693536$

$-0.1766612259 \quad 0.0441488668$

$0.2988530375 \quad 1.3433022421$

$0.9568393255 \quad 2.3979495495$

$-0.0211063476 \quad 3.3570968967$

$-2.4818991002-0.2772005779$ 

3.6941416509
2.1497080524
3.4204075574
4.8959391336
4.0779775269
6.2899914640
5.0499103904
5.8767847236
7.2495097486
6.8216688428
5.4823524377
7.9058488113
7.6903073812
5.7639602497
5.8489243508
$-0.5776488179$
$-1.2654332619$
$-1.4250182174$
$-0.9022260820$
$-0.2137099254$
$-0.4700020085$
$-1.6856523130$
$-1.9726821742$
$-1.0452784908$
0.1482582046

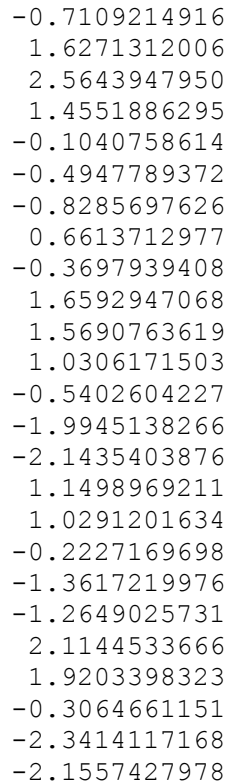
$-2.1557427978$

$\mathrm{II}_{6}$

Geometry:

37

\begin{tabular}{lr}
$\mathrm{Cl}$ & 0.0129651989 \\
$\mathrm{C}$ & 0.0072348197 \\
$\mathrm{Pd}$ & 1.5964090733 \\
$\mathrm{P}$ & 2.8356310574 \\
$\mathrm{P}$ & 3.1379251430 \\
$\mathrm{C}$ & 4.5334913049 \\
$\mathrm{C}$ & 4.9674650858 \\
$\mathrm{C}$ & 5.7294543969 \\
$\mathrm{C}$ & 6.5642560025 \\
$\mathrm{C}$ & 5.7766606533 \\
$\mathrm{C}$ & 5.4447551534 \\
$\mathrm{H}$ & 2.7624710062 \\
$\mathrm{H}$ & 2.6840642741 \\
$\mathrm{H}$ & 2.2026164452 \\
$\mathrm{H}$ & 3.0292634459 \\
$\mathrm{H}$ & 4.9916321450 \\
$\mathrm{H}$ & 4.3754911282 \\
$\mathrm{H}$ & 6.3816181189 \\
$\mathrm{H}$ & 4.9683599977 \\
$\mathrm{H}$ & 5.0335415618 \\
$\mathrm{H}$ & 6.4192065281 \\
$\mathrm{H}$ & 6.3557047085 \\
$\mathrm{H}$ & 4.8551938512 \\
$\mathrm{H}$ & 7.1405382537 \\
$\mathrm{H}$ & 7.3077295715 \\
$\mathrm{H}$ & 5.4588237289 \\
$\mathrm{H}$ & 5.0012156789 \\
$\mathrm{C}$ & -0.4882551637 \\
$\mathrm{C}$ & -1.6190364333 \\
$\mathrm{C}$ & -2.2551742254 \\
$\mathrm{C}$ & -1.7594930710 \\
$\mathrm{C}$ & -0.6320247266 \\
$\mathrm{H}$ & -0.0192318020 \\
$\mathrm{H}$ & -2.0028104771 \\
$\mathrm{H}$ & -3.1334724527 \\
$\mathrm{H}$ & -2.2558407042 \\
$\mathrm{H}$ & -0.2766183077 \\
& \\
\hline
\end{tabular}

\section{$\mathrm{II}_{\mathrm{SH}, 6}$}

Geometry:

Cl $\quad 0.1386599159$

C $\quad 0.0297254952$

$\mathrm{Pd} \quad 1.6140745631$

P $\quad 3.0125489432$

P $\quad 3.4442309469$

C $\quad 3.1184532529$

C $\quad 3.6849958230$
29 Total energy: $-0.0254902631$ 0.0028060937 0.0464875381 0.0641905663 $-0.7034781050$ 0.1511284437 1. 4035686166 1. 2542708463 1.0025091813 $-0.4740046581$ $-0.9966534102$ 1. 1302217101 $-0.4989783252$ 1. 3575327520 $-0.3180142305$ $-1.7791180924$ $-1.0264592781$ $-0.9248395186$ 2. 2463204416 1. 7108368303 1.3822874029 1. 6014940880 2. 1816951662 0.4548568585 $-0.7663204825$ 0.1185593971 1. 2156279546 1. 2309078665 0.0357352998 $-1.1753100326$ $-1.1945528725$ 2.1565888069 2. 1807392711 0.0472203305 $-2.1105969660$ $-2.1413404038$

18 Total energy: $-0.1439042202$ $-0.0631740319$ $-0.0362571938$ $-0.1888574703$ $-0.0015716619$ $-1.9339171318$ $-1.4584821622$ $-0.0045189268$
$-1.3480909520$

4. 3542279241

2. 9634863027

4. 8422446076

4.9625962947

3.9961820545

2. 7955295041

$-0.3694250313$

$-0.6895975647$

3. 0006753514

1.8740092807

1. 0488397951

1.7962869988

1.0635189390

$-0.6800694523$

2.6486063683

3. 8544532758

4. 4709634705

3. 8532440021

2. 6370746005

2.1622509829

4.3151020533

5. 4051446140

4. 3024370432

2.1342432799

$-1739.61983581491$

0.0131183880

3. 1003782614

1. 8276463087

3. 7838802986

$-0.1757470474$

3. 9509958567

$-0.6138472093$

$-0.1151455926$

1. 1719383656

2. 4723562975

2.7387991119

$-1.0278769281$

$-0.9817381646$

4. 9188341721

4. 2801237271

4. 8710028062

4.0968879209

2. 8977878609

1.8632855431

0.0083099008

$-0.9121636407$

3. 3262875120

2. 4433402607

1. 2885554246

1. 0325750982

$-0.2695446302$

$-1.7090085005$

3. 5891103381

4. 4182211982

4. 7635102423

4.2703358406

3. 4392206996

3. 3122121014

4. 7836779665

5.4037773800

4. 5205852510

3. 0420841164

$-2138.48851462610$

$-0.0405380951$

3.2261165477

1. 9202051671

3. 7513773888

0.2959761657

4. 4113746957

$-0.8546210885$ 


$\begin{array}{lr}\mathrm{C} & 4.4736977141 \\ \mathrm{C} & 3.8242978696 \\ \mathrm{C} & 4.1715774587 \\ \mathrm{C} & 3.0431683419 \\ \mathrm{H} & 3.2594299800 \\ \mathrm{H} & 4.7750094567 \\ \mathrm{H} & 2.6280532803 \\ \mathrm{H} & 4.4177335093 \\ \mathrm{H} & 4.0559901587 \\ \mathrm{H} & 2.2858445165 \\ \mathrm{H} & 3.0436265059 \\ \mathrm{H} & 2.0758599624 \\ \mathrm{H} & 5.4912367252 \\ \mathrm{H} & 4.5808819258 \\ \mathrm{H} & 5.0927115117 \\ \mathrm{H} & 4.4314714383 \\ \mathrm{H} & 4.1289990094 \\ \mathrm{H} & 2.7340186052 \\ \mathrm{H} & 2.6750829029 \\ \mathrm{H} & 4.1950928416 \\ \mathrm{C} & -0.2541790986 \\ \mathrm{C} & -1.3827463468 \\ \mathrm{C} & -2.2466209118 \\ \mathrm{C} & -1.9771730024 \\ \mathrm{C} & -0.8566416620 \\ \mathrm{H} & 0.4043776281 \\ \mathrm{H} & -1.5810635445 \\ \mathrm{H} & -3.1185344259 \\ \mathrm{H} & -2.6468277196 \\ \mathrm{H} & -0.6882205105 \\ \mathrm{~S} & 6.4912746919 \\ \mathrm{H} & 6.1483958847 \\ & \\ \mathrm{H} & \end{array}$

$\mathrm{TS}_{\mathrm{SH}, 6}^{\mathrm{SH}-\mathrm{Cl}}$

Geometry:

Cl $\quad 0.0932161185$

C $\quad-0.0479634382$

$\mathrm{Pd} \quad 1.5818681767$

P $\quad 2.5881450428$

P $\quad 3.2868034393$

1. 9416622229

2. 9884551731

3.1703159749

2.1962475148

2. 6729774144

1. 5325072770

3.4811454925

4. 6523744126

2.5762440564

3.9791786936

2. 6970149919

1.0654752080

1.0921480778

0.7365952347

4.2076440780

3.0432953118

3. 2955554376

3.3391429096

2.0357848588

1.2210319509

1. 9587004770

3. 6421550956

$-0.1496232592$

$-1.2683893436$

$-2.3051360180$

$-2.2233595214$

$-1.1072208270$

0.6403257905

$-1.3290128980$

$-3.1684637373$

$-3.0335588358$

$-1.0899781453$

3. 1213737217

2.0095408885
$-2.6755148134$

$-3.4308446070$

$-2.8401566878$

$-2.9723098760$

1.0273733023

0.2826912870

0.5287438361

0.1995851689

$-2.0235208519$

$-2.0807413312$

$-3.9828972243$

$-2.8467649697$

$-2.3665932344$

$-3.3651085247$

$-3.3003455946$

$-1.7827695872$

$-4.4868218548$

$-3.4256230251$

$-1.7630739552$

$-1.0779614207$

1.0022712945

0.9836317881

$-0.1155220323$

$-1.1849141747$

$-1.1537113070$

1.8672193877

1.8280122052

$-0.1375931362$

$-2.0431804527$

$-1.9781321258$

0.6047903647

1. 9155605681

61 Total energy: 0.6363416965 $-0.2396979295$ $-0.0435360912$ $-1.4635841000$ $-0.0908066029$ $-3.2307181163$ $-1.1362729573$ $-2.6717024807$ $-3.4827131291$ $-3.6581048837$ $-3.6912913472$ 1. 1584913044 $-0.3945961345$ $-1.1545824225$ $-1.7053234448$ $-3.8908834627$ $-3.2340224765$ $-4.6984272097$ $-3.0232927250$ $-2.9297891821$ $-2.9959900029$ $-4.5613793919$ $-2.8201334067$ $-4.4720987966$ $-2.9794451077$ $-0.8864119418$ $-0.7283785290$ 0.4574814597 0.2900071618 $-0.5736325137$ $-1.2525936033$ $-1.0817960301$ 1.1512876057 0.8414120616 $-0.7118221806$ $-1.9089614169$ $-1.5669397670$ 2. 4947574377 2. 8999883294
$-0.3227453892$

0.8555835662

2.2396797077

3. 2785954594

$-0.6545989323$

0.7080629477

4.9093517969

3. 5511924494

4. 9762616464

5.1090667670

3.7139168806

2.7763856209

$-0.0473564918$

$-1.1730624702$

2. 6232207501

2. 1217458724

0.8200956551

0.7132047380

$-1.1587183772$

$-1.7492438594$

4.0962758083

4. 9287929341

4.9200539787

4.0591153297

3.2191224317

4.1300739795

5.5870774114

5.5712162134

4.0320555093

2.5318970556

2. 9634408875

2. 8828877470

$-2138.46650750234$

0.1174934517

3.2971848162

2. 0581807886

3. 5359685084

0.3089072622

3. 5691068693

$-1.2326400593$

$-1.2164436606$

$-0.3342469197$

1.1249800469

2.1603027368

$-0.3116437622$

0.5738120901

4. 9140314576

3.3970754257

4.0170879325

4. 2272926027

2.2168066940

1.8167016073

$-0.9510895668$

$-2.2601965902$

1. 2144790238

1.3607315990

$-0.7863869550$

$-0.3411660688$

$-1.5161513051$

$-2.0149878117$

4.5103763162

5. 3406658547

4. 9702583819

3. 7487842747

2. 9196152182

4. 7906536418

6.2780074526

5. 6191023367

3. 4321043285

1. 9477586029

2. 8420068351

2.1806980644

II ' $\mathrm{Cl}, 6$ 
39

$\begin{array}{lrrrr}\text { Geometry: } & \text { 20 Total } & \text { energy: } & -2138.50586376618 \\ \mathrm{~S} & 0.0176812043 & -0.1152611147 & 0.0253975502 \\ \mathrm{C} & -0.0081771002 & -0.0126219038 & 3.1609279534 \\ \mathrm{Pd} & 1.5706620501 & -0.0874706427 & 1.8497736307 \\ \mathrm{P} & 3.0209772365 & -0.1259275547 & 3.6912068823 \\ \mathrm{P} & 3.3972285991 & -0.0772839332 & 0.2348081578 \\ \mathrm{C} & 3.5156988075 & -1.8502658450 & 4.1942303037 \\ \mathrm{C} & 4.1202993107 & -1.6523907684 & -0.5016607297 \\ \mathrm{C} & 5.5703606986 & -2.0285892898 & -0.1004460432 \\ \mathrm{C} & 5.7628787689 & -2.9420084012 & 1.1283563498 \\ \mathrm{C} & 5.4007402635 & -2.3273380517 & 2.4981983230 \\ \mathrm{C} & 3.9938766779 & -2.6948140440 & 2.9991571972 \\ \mathrm{H} & 3.0436827116 & 0.6156350738 & -0.9455751609 \\ \mathrm{H} & 4.5656912962 & 0.6382746701 & 0.6072988007 \\ \mathrm{H} & 2.5444014088 & 0.3965018219 & 4.9163223604 \\ \mathrm{H} & 4.2612541306 & 0.5586078118 & 3.5477539634 \\ \mathrm{H} & 4.2919939097 & -1.7953240009 & 4.9686310748 \\ \mathrm{H} & 2.6253869859 & -2.3043189593 & 4.6460385828 \\ \mathrm{H} & 3.9872216066 & -3.7561191536 & 3.2918820267 \\ \mathrm{H} & 3.2567907800 & -2.6004263624 & 2.1913244791 \\ \mathrm{H} & 6.1660100142 & -1.1182041057 & 0.0441514310 \\ \mathrm{H} & 6.0115023526 & -2.5530909911 & -0.9616177512 \\ \mathrm{H} & 6.1254059898 & -2.6652898747 & 3.2528807902 \\ \mathrm{H} & 5.5415662726 & -1.2414165248 & 2.4434572190 \\ \mathrm{H} & 6.8258731104 & -3.2192868933 & 1.1401528935 \\ \mathrm{H} & 5.2083135706 & -3.8821143716 & 0.9758986320 \\ \mathrm{H} & 3.4297982306 & -2.4752553461 & -0.2824466236 \\ \mathrm{H} & 4.0870928064 & -1.5081932757 & -1.5874961503 \\ \mathrm{C} & -0.4888401605 & 1.2225750132 & 3.6286564463 \\ \mathrm{C} & -1.5773401784 & 1.2947672378 & 4.5066703292 \\ \mathrm{C} & -2.2102351930 & 0.1267929646 & 4.9452748846 \\ \mathrm{C} & -1.7424004422 & -1.1102845918 & 4.4923429365 \\ \mathrm{C} & -0.6548175682 & -1.1757836205 & 3.6108452880 \\ \mathrm{H} & -0.0187780232 & 2.1444333095 & 3.2939137951 \\ \mathrm{H} & -1.9324912105 & 2.2668990034 & 4.8451552999 \\ \mathrm{H} & -3.0565075986 & 0.1809504956 & 5.6266666377 \\ \mathrm{H} & -2.2269788936 & -2.0289985043 & 4.8194526865 \\ \mathrm{H} & -0.3201683557 & -2.1488582372 & 3.2582661496 \\ \mathrm{H} & -1.1433101106 & -0.1725397448 & 0.7129413972 \\ \mathrm{Cl} & 6.2265954253 & 1.2657033917 & 2.3331496978\end{array}$

II ' 6

Geometry: 16 Total energy: -1678.19819593256

$\begin{array}{lrrr}\mathrm{S} & -0.0558898232 & 0.0143750957 & -0.0144175882 \\ \mathrm{C} & -0.0069538643 & 0.0111474570 & 3.0912912003 \\ \mathrm{Pd} & 1.5391406213 & 0.0121637979 & 1.7361999698 \\ \mathrm{P} & 2.9581620774 & -0.0618432079 & 3.6159781970 \\ \mathrm{P} & 3.2875142453 & -0.0289401582 & 0.0132583885 \\ \mathrm{C} & 3.4939015408 & -1.7767690849 & 4.1070213159 \\ \mathrm{C} & 4.0215055838 & -1.6197470483 & -0.6799550606 \\ \mathrm{C} & 5.4792215728 & -1.9773824292 & -0.2973312208 \\ \mathrm{C} & 5.7133085821 & -2.7934152964 & 0.9883114019 \\ \mathrm{C} & 5.3295033486 & -2.1022969484 & 2.3174794847 \\ \mathrm{C} & 3.9968364855 & -2.5947561229 & 2.9067183013 \\ \mathrm{H} & 2.8414815732 & 0.5852753579 & -1.1779203286 \\ \mathrm{H} & 4.4719786249 & 0.7380663427 & 0.1695312821 \\ \mathrm{H} & 2.4691359476 & 0.4423838432 & 4.8398622992 \\ \mathrm{H} & 4.1922484875 & 0.6370915135 & 3.6017238460 \\ \mathrm{H} & 4.2510935183 & -1.7220131415 & 4.8992497237 \\ \mathrm{H} & 2.6043812617 & -2.2524574318 & 4.5363201049 \\ \mathrm{H} & 4.1193231837 & -3.6405113433 & 3.2213726144 \\ \mathrm{H} & 3.2177856745 & -2.5960583911 & 2.1336057315 \\ \mathrm{H} & 6.0891220758 & -1.0635854684 & -0.2599369387 \\ \mathrm{H} & 5.8877567118 & -2.5681775217 & -1.1285286109 \\ \mathrm{H} & 6.1178028979 & -2.2679174699 & 3.0647378242 \\ \mathrm{H} & 5.3013996618 & -1.0146086528 & 2.1624276488 \\ \mathrm{H} & 6.7806744600 & -3.0493462995 & 1.0089864240 \\ \mathrm{H} & 5.1809441016 & -3.7523102950 & 0.9066719656 \\ \mathrm{H} & 3.3401653911 & -2.4352949886 & -0.4132754128 \\ \mathrm{H} & 3.9662548350 & -1.5182592972 & -1.7683172834 \\ \mathrm{C} & -0.4507408852 & 1.2080177910 & 3.6730467664 \\ \mathrm{C} & -1.4722733121 & 1.2007582250 & 4.6320559558 \\ \mathrm{C} & -2.0641160212 & -0.0042785923 & 5.0247357138 \\ \mathrm{C} & -1.6308046675 & -1.2008725424 & 4.4467103602 \\ \mathrm{C} & -0.6100009833 & -1.1928371702 & 3.4852440314\end{array}$




$\begin{array}{lrr}-0.0099541944 & 2.1561841915 & 3.3741762161 \\ -1.8062498219 & 2.1398114015 & 5.0690611615 \\ -2.8566888887 & -0.0094564894 & 5.7688740700 \\ -2.0895711366 & -2.1443308823 & 4.7363511925 \\ -0.2992504384 & -2.1321832969 & 3.0339686189 \\ -1.1965490760 & -0.0832501653 & 0.6991877648\end{array}$

$\mathrm{TS}_{\mathrm{SH}, 6}{ }^{\mathrm{RED}}$
38

Geometry: 13 Total energy: -1678.16368521768

$\begin{array}{lrrr}\mathrm{S} & 0.0850318230 & -0.1924901835 & -0.0696492937 \\ \mathrm{C} & 0.0234510178 & -0.0072075391 & 2.1029873812 \\ \mathrm{Pd} & 2.0199312611 & -0.0864872648 & 1.3631845722 \\ \mathrm{P} & 3.2295226751 & -1.0221529826 & 3.2641710433 \\ \mathrm{P} & 3.7993208122 & 0.9263962173 & -0.0334048528 \\ \mathrm{C} & 3.9217124858 & -2.7638151533 & 3.0437460653 \\ \mathrm{C} & 4.4707990909 & 0.1482004762 & -1.6078449262 \\ \mathrm{C} & 5.4602627623 & -1.0269632829 & -1.4531441189 \\ \mathrm{C} & 4.9090016717 & -2.2725658470 & -0.7278103589 \\ \mathrm{C} & 5.0795147834 & -2.2320482777 & 0.8023680483 \\ \mathrm{C} & 4.0841219147 & -3.1266146035 & 1.5574891637 \\ \mathrm{H} & 3.3853962488 & 2.1636421513 & -0.5956549114 \\ \mathrm{H} & 5.0228750741 & 1.4263854618 & 0.4985340048 \\ \mathrm{H} & 2.6409814309 & -1.1589515107 & 4.5479482785 \\ \mathrm{H} & 4.3932264824 & -0.3433488337 & 3.7189849319 \\ \mathrm{H} & 4.8690097959 & -2.8636675315 & 3.5865979975 \\ \mathrm{H} & 3.2083258476 & -3.4502590731 & 3.5148123993 \\ \mathrm{H} & 4.3824769940 & -4.1811204618 & 1.4699049855 \\ \mathrm{H} & 3.0999743947 & -3.0372368298 & 1.0781946146 \\ \mathrm{H} & 6.3758382957 & -0.6785039521 & -0.9530936792 \\ \mathrm{H} & 5.7644064579 & -1.3086536220 & -2.4705459007 \\ \mathrm{H} & 6.1120770987 & -2.4987865314 & 1.0712749825 \\ \mathrm{H} & 4.9402430961 & -1.1987562582 & 1.1414838400 \\ \mathrm{H} & 5.3990224092 & -3.1723924780 & -1.1230093407 \\ \mathrm{H} & 3.8425573168 & -2.3766476061 & -0.9711428942 \\ \mathrm{H} & 3.5885546346 & -0.1894426712 & -2.1673798300 \\ \mathrm{H} & 4.9434108606 & 0.9393713248 & -2.2012009427 \\ \mathrm{C} & -0.1883669042 & 1.3062084770 & 2.5705909948 \\ \mathrm{C} & -0.8799716947 & 1.5311386879 & 3.7661613878 \\ \mathrm{C} & -1.4121609665 & 0.4642210989 & 4.4951468271 \\ \mathrm{C} & -1.2433705385 & -0.8403480029 & 4.0098671430 \\ \mathrm{C} & -0.5374732755 & -1.0787136586 & 2.8305755363 \\ \mathrm{H} & 0.1753484911 & 2.1530628227 & 1.9956217629 \\ \mathrm{H} & -1.0147202249 & 2.5520792733 & 4.1172915469 \\ \mathrm{H} & -1.9598381862 & 0.6428769203 & 5.4164427622 \\ \mathrm{H} & -1.6533893461 & -1.6826485607 & 4.5636538413 \\ \mathrm{H} & -0.4049855322 & -2.1005621930 & 2.4831667965 \\ \mathrm{H} & -0.4407869890 & -1.4201772766 & 0.1231242635\end{array}$

$\mathrm{C}_{\mathrm{SH}, 6}$

Geometry:

\begin{tabular}{|c|c|c|c|}
\hline & Cy: & 0 Total energy: & -1678.1995 \\
\hline S & 0.4785713885 & 0.0001272763 & -0.1414974643 \\
\hline C & -0.3516728277 & 0.2009852226 & 1.4292898689 \\
\hline $\mathrm{Pd}$ & 4.2294657196 & -0.2577316807 & 2.8403613070 \\
\hline $\mathrm{P}$ & 4.0910143051 & -2.5588230350 & 3.1448583578 \\
\hline $\mathrm{P}$ & 4.8476436747 & 1.7114375571 & 1.7792864764 \\
\hline $\mathrm{C}$ & 5.2241296635 & -3.5426802501 & 2.0204135533 \\
\hline C & 5.0770764441 & 1.7234928159 & -0.0824235217 \\
\hline C & 6.0159234795 & 0.6565535249 & -0.6944360554 \\
\hline C & 5.4727454735 & -0.7947164729 & -0.7276334635 \\
\hline C & 6.0078910740 & -1.7048556814 & 0.3914448998 \\
\hline $\mathrm{C}$ & 5.2183884468 & -3.0153111850 & 0.5711736830 \\
\hline $\mathrm{H}$ & 4.1135616036 & 2.9246193115 & 1.8869098378 \\
\hline $\mathrm{H}$ & 6.0968406073 & 2.2720939454 & 2.1647761642 \\
\hline $\mathrm{H}$ & 2.8654364323 & -3.2296504179 & 2.8849609455 \\
\hline $\mathrm{H}$ & 4.3595084725 & -3.2280119544 & 4.3691649160 \\
\hline $\mathrm{H}$ & 6.2340260484 & -3.4789183193 & 2.4448064697 \\
\hline $\mathrm{H}$ & 4.9209772690 & -4.5948017440 & 2.0528818691 \\
\hline $\mathrm{H}$ & 5.6266944912 & -3.7950888330 & -0.0869730910 \\
\hline $\mathrm{H}$ & 4.1791567211 & -2.8629720832 & 0.2487350221 \\
\hline $\mathrm{H}$ & 6.9923905189 & 0.6801387831 & -0.1893705161 \\
\hline $\mathrm{H}$ & 6.2100407807 & 0.9826526303 & -1.7248363212 \\
\hline $\mathrm{H}$ & 7.0690299825 & -1.9345885629 & 0.2123431857 \\
\hline $\mathrm{H}$ & 5.9640897165 & -1.1433746425 & 1.3345661430 \\
\hline $\mathrm{H}$ & 5.7065166610 & -1.2476684333 & -1.7015286430 \\
\hline $\mathrm{H}$ & 4.3766569091 & -0.7628576019 & -0.6658380159 \\
\hline
\end{tabular}




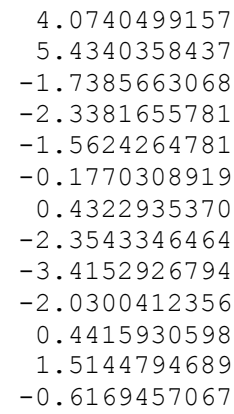

$1_{\mathrm{v}, \mathrm{Cl}}$
1. 6161569864 2. 7239022520 0.3743651561 0.5243926299 0.5018979620 0.3283526941 0.1789033550 0.3925680427 0.6586345391 0.6184252927 0.3098128965 0.0471373616 0.1547071755

3 Total energy: 0.0056993173 $-0.0133879736$ $-0.0301520078$ 0.0417503653 $-1.1754234059$ $-2.0155412652$ $-1.2010125921$ $-2.7369284175$ $-2.0020474880$ 1.5994107300 1.8245746609 1.0331289014 2.8148268203 2. 4311051429 $-1.6207158324$ $-1.9044288963$ $-1.1479015107$ $-2.9100879326$ $-2.4186708764$ 1. 1311249243 1. 9423447571 1. 9394319970 2. 6290123745 1. 1460556677 0.2299076583 0.3384222674 0.2838746155 0.4768865296 0.2877711821 $-0.1579145617$ $-0.2440962820$ $-0.1964227365$ $-0.3495991726$ $-0.2101193575$
$-0.5138167550$

$-0.3547325232$

1. 5268361557

2. 7797294991

3. 9433119601

3.8392844114

2. 5902648111

0.6307750149

2.8404775241

4.9170335971

4.7331035958

2. 5324173521

$-0.9155076106$

$-1737.73219919173$

0.8305031947

3.4638316049

$-0.0674817994$

$-0.1864116177$

$-1.5167693815$

$-2.0029126076$

$-1.9102361684$

$-2.7851319361$

$-1.6274909178$

$-1.0461384205$

$-2.3660676987$

$-3.0895446955$

$-2.7697537064$

$-0.3598987413$

$-0.8273987311$

$-2.1305822311$

$-2.8981881500$

$-2.4765556803$

$-0.0950898350$

$-1.4250856659$

$-2.0000654293$

$-1.6828507111$

$-2.7996491159$

$-1.7605844034$

1.0240406629

2.3493668763

0.5690787630

2. 9996080103

2.8229136147

0.8256285737

2.1586886834

0.3160232307

2. 7607317958

2.6865497764

\section{$1_{\mathrm{v}}$}

Geometry:

$\begin{array}{lr}\text { C } & 1.1599777392 \\ \text { P } & 1.2924985294 \\ \text { C } & 3.1117274918 \\ \text { C } & 3.6767547678 \\ \text { C } & 0.3484526634 \\ \text { Pd } & 0.0000526212 \\ \text { P } & -1.2943169468 \\ \text { C } & -1.1592000376 \\ \text { C } & -0.3548845517 \\ \text { C } & 1.0082920325 \\ \text { C } & 0.0993138958 \\ \text { C } & -1.0133015786 \\ \text { C } & -0.0961095977 \\ \text { C } & -3.1139870517 \\ \text { C } & -3.6783947078 \\ \text { H } & -1.7594905401 \\ \text { H } & -0.2728595623 \\ \text { H } & 0.2482247952 \\ \text { H } & -3.7374314508 \\ \text { H } & -4.7577496651 \\ \text { H } & -3.0681700062 \\ \text { H } & -1.6194588305\end{array}$

3 Total energy: $-1.6842648661$ $-1.5972460384$ $-1.4832972055$ $-0.5226492266$ $-0.8734726880$ $-0.0009394186$ 1.5964936735 1.6931232252 0.8786749611 $-3.3535016480$ $-3.7077693378$ 3.3503005497 3. 7015040083 1. 4793422201 0.5213134205 2. 4408881897 0.9321387518 0.1295003706 2. 2202083798 0.4483017183 $-0.2221215752$ 4. 1146126788
$-1277.45576488257$

$-2.9093850436$

$-1.0819508330$

$-0.8778638132$

$-0.1417052005$

$-3.5939477563$

0.0013705413

1. 0808579145

2. 9076688238

3.5962955445

$-0.6357210478$

0.2768315359

0.6246784580

$-0.2806192240$

0.8794012750

0.1392494279

3. 4303991574

4.6804839894

3. 0871839876

1.3844795239

0.0178909927

$-0.3696530375$

1. 1157333793 

0.0740134321
4.7413385758
$-0.5542149630$
0.5145390239
1.7663396984
0.2656179845
$-0.2603113201$
1.6064616888
$-0.0714949885$
$-0.5033404937$
3.7345873153
4.7559740990
2.9493302809
$-0.7761114042$
$-2.4245558289$
$-3.4357885413$
$-0.9234541301 \quad-4.6782553489$
$-0.1313548950-3.0812938543$
$-4.1166001320 \quad-1.1383411092$
$\begin{array}{ll}-4.7488462641 & 0.5452105852\end{array}$
$\begin{array}{ll}-2.9569943572 & 0.7840270323\end{array}$
$-2.2280379402-1.3779507725$
$-0.4522482829 \quad-0.0175977903$
$\begin{array}{ll}3.0672247974 & 0.2257409669\end{array}$
0.3606858228

\section{$\mathrm{C}_{\mathrm{v}}$}

45

Geometry: 11 Total energy: -1969.29460111760

C $\quad 0.2030377827$

1.5273507351

$-0.6004133619$

$-1.6270180327$

$-1.5101442497$

$-1.8705570281$

$-1.9439481501$

0.7333464698

1.9531759166

3.3084640830

3.6188358403

0.9986506786

$-0.3200920410$

2.8369942443

2.8094855288

1.5375362253

$-0.8901352352$

$-0.8719741681$

3.8666114058

4.4224855332

3.0706147589

3. 3971193373

3. 3337768255

2.2552820980

$-0.4303664685$

2.0100604956

2.1686017844

$-2.3399788788$

$-2.1084286569$

$-0.8040155262$

$-2.5656377667$

$-2.6824732705$

$-1.2564175035$

$-4.4812973897$

$-3.8996442929$

$-4.0071820921$

$-4.6874020773$

$-5.2636120803$

$-5.1645094507$

$-3.3670135327$

$-3.5523586386$

$-4.7665862017$

$-5.7935870513$

$-5.6072822197$

energy: $\quad-1969.2$
-0.2537918065

$-0.3240117866$

$-0.3911834037$

1.3940721157

$-0.2105594329$

1.3453897298

$-0.2478315049$

2.2740708602

$-1.7693558116$

$\begin{array}{ll}1.8361592435 & 2.1476682283\end{array}$

$\begin{array}{ll}3.2733476874 & 0.0257985272\end{array}$

$5.2466234033 \quad 0.2555984228$

$5.5457809539-0.9462103178$

$4.6547657637-1.8993813419$

$\begin{array}{rr}6.8104213519 & 0.1069249867\end{array}$

$6.8126175213 \quad-0.1333207391$

$5.5241792899 \quad 1.8454981477$

$4.6240483126 \quad 2.8330666361$

$\begin{array}{ll}7.7553780992 & 0.2164079175\end{array}$

$7.7392579660 \quad-0.2260864085$

$5.8802922410 \quad-0.2462392961$

$6.4823269808-0.8662247159$

$4.8305018263-2.6134518773$

$3.7176441411-1.9912617041$

$6.4538578868 \quad 1.9708525360$

$4.7852109686 \quad 3.7770247005$

$\begin{array}{ll}3.6933901799 & 2.7212538344\end{array}$

$-1.1319250808 \quad-0.4646894960$

$-1.3660291975-0.2536034609$

$0.4769083541-0.0707939136$

$0.5268065306-1.8878461934$

$2.2434065350 \quad-3.6335698671$

$3.0957746602-2.6200530214$

$0.2563583296 \quad 0.8990222999$

$1.6447190831 \quad 2.9246296511$

$2.6656707797 \quad 2.3042415754$

$5.9796046282-1.7468487080$

$4.9449549027-1.0139684624$

$4.9602052988 \quad 0.3799821975$

$5.9942095865 \quad 1.0256217352$

$\begin{array}{rr}7.0205564962 & 0.2738301179\end{array}$

$7.0204348165-1.1201342126$

$4.1453039700 \quad-1.5171290837$

$4.1569686849 \quad 0.9524733907$

$6.0009838836 \quad 2.1094552371$

$\begin{array}{ll}7.8286231490 & 0.7691488445\end{array}$

$7.8142455259-1.7121872006$

$5.9749364468 \quad-3.5071306336$

$\mathrm{TS}_{\mathrm{v}}{ }^{\mathrm{ox}}$

\begin{tabular}{lr}
\multicolumn{2}{c}{45} \\
Geometry: \\
C & -0.0750099472 \\
C1 & -0.0912464512 \\
C & 1.2317524558 \\
C & -1.0907169200 \\
C & -0.8160196371 \\
C & 0.4630468735 \\
C & 1.4809288779 \\
Pd & -0.2961709315 \\
P & -1.9865544101 \\
C & -3.5591637639 \\
& \\
C & -3.9099292181 \\
P & 0.7991176683
\end{tabular}

24 Total energy:

$-0.0163378432$

0.1350027029

$-0.0788638676$

$-0.8278528440$

$-1.6059151736$

$-1.6177544525$

$-0.8551815713$

2.0414637805

2.6958627448

1. 7605466364

$-1969.25548235979$

0.1454751724

2. 2703263112

$-0.3738454026$

$-0.3894002372$

$-1.5123466741$

$-2.0898720432$

$-1.5136478097$

0.5886336770

$-1.0087235730$

$-1.0300489832$

0.8373854415

$-1.9329343630$

1. 3562916308 


$$
\begin{array}{r}
2.1597399097 \\
2.5355321181 \\
-1.5367587162 \\
-0.4670212401 \\
-2.6587143760 \\
-2.3207401577 \\
1.6162143068 \\
1.6389108062 \\
-0.2764399334 \\
-0.3215698308 \\
2.0992505529 \\
2.1318759986 \\
1.1606547236 \\
-0.9681665738 \\
-1.0354573652 \\
0.3393722579 \\
2.6725906748 \\
3.3567233004 \\
2.0303109842 \\
-2.2122292359 \\
-0.2457073527 \\
0.2141513148 \\
-3.3578085051 \\
-2.7417424383 \\
-1.6141150141 \\
-4.2033749384 \\
-4.8388913447 \\
-3.2847464747 \\
-2.0831349837 \\
-1.6144813272 \\
0.6648167797 \\
2.4858725893 \\
2.0375204584
\end{array}
$$

\section{$I I_{\mathrm{v}}$}

Geometry:

$\begin{array}{lrr}\mathrm{C} & 2.0671472211 & 0.5845237475 \\ \mathrm{C} & 1.9240436523 & -0.0801694782 \\ \mathrm{C} & 3.0404317838 & -0.6903144766 \\ \mathrm{C} & 4.2856323769 & -0.6447164749 \\ \mathrm{C} & 4.4233920590 & 0.0056097341 \\ \mathrm{C} & 3.3111291722 & 0.6228663230 \\ \mathrm{Pd} & 0.1293263537 & -0.0328138110 \\ \mathrm{Cl} & 0.8027424805 & 2.1788860344 \\ \mathrm{P} & -0.3483819677 & -2.2343421261 \\ \mathrm{C} & 0.7476614154 & -3.3475490169 \\ \mathrm{C} & 1.6826766579 & -2.9916123472 \\ \mathrm{P} & -2.0114586479 & 0.4471807159 \\ \mathrm{C} & -1.7927631937 & 0.9595299527 \\ \mathrm{C} & -0.5995455544 & 1.0585943812 \\ \mathrm{C} & -1.8951086501 & -2.4543414871 \\ \mathrm{C} & -2.5529390400 & -1.4420096976 \\ \mathrm{C} & -0.5675447959 & -3.2882227128 \\ \mathrm{C} & -1.6874420867 & -3.9111304607 \\ \mathrm{C} & -2.9518806858 & 1.8357836754 \\ \mathrm{C} & -2.4593240995 & 2.6412962786 \\ \mathrm{C} & -3.3961831559 & -0.7456018907 \\ \mathrm{C} & -3.7977653804 & -1.3444976057 \\ \mathrm{H} & -3.9662425194 & 1.9822248222 \\ \mathrm{H} & -3.0588737735 & 3.4525996333 \\ \mathrm{H} & -1.4390561541 & 2.5452127742 \\ \mathrm{H} & -3.8978211205 & -0.9782685924 \\ \mathrm{H} & -4.6221973548 & -2.0551320277 \\ \mathrm{H} & -3.3189997843 & -1.1585809971 \\ \mathrm{H} & -2.7040198897 & 1.2020604940 \\ \mathrm{H} & -0.5127004955 & 1.3791364891 \\ \mathrm{H} & 0.3228982696 & 0.8650266036 \\ \mathrm{H} & -2.2284353163 & -3.4814807984 \\ \mathrm{H} & -3.4329453012 & -1.6137788077 \\ \mathrm{H} & -2.2343788942 & -0.4099219999 \\ \mathrm{H} & 0.3303692527 & -3.3669467702 \\ \mathrm{H} & -1.7248647553 & -4.4996489446 \\ \mathrm{H} & -2.6088500103 & -3.8482647172 \\ \mathrm{H} & 0.5390865949 & -4.4054422378 \\ \mathrm{H} & 2.2346742853 & -3.7531301979 \\ & 1.9355816674 & -1.9581455165\end{array}$

2. 5714744402

3. 0863882397

$-2.7811510693$

$-3.2789601215$

$-0.7249044425$

$-1.3830145478$

0.0893054551

$-1.2099970528$

2.1524440175

3. 4602018840

0.4417174060

$-1.9375661361$

$-1.5907673598$

1. 4648452529

3. 8631773604

4.1762001812

2.8636446564

3. 7970597097

2. 8262546697

$-3.4401236025$

$-4.3446933229$

$-2.6464300587$

0.1103556340

$-1.1115170966$

$-2.2090946469$

$-0.1696359510$

$-1.8399222632$

$-2.7922531762$

0.0465933112

$-1.9467341490$

$-2.9612725284$

$-1.9292373192$

0.1078533276

\footnotetext{
$-1969.29555138115$

$-2.2637734751$

$-1.0397127587$

$-0.4590198128$

$-1.0984777779$

$-2.3271459499$

$-2.9066310438$

$-0.0778723811$

0.5899342977

$-0.7357900806$

$-1.7027252174$

$-2.5866169656$

1. 1827336336

2. 9209058584

3. 5121923325

$-1.6892404540$

$-2.2619619131$

0.7418820771

1. 1178582118

0.4365142372

$-0.5087583264$

1. 3423582788

2. 4685189371

0.8107515789

$-0.9187106960$

$-0.8673519378$

0.4035626897

2. 4683012607

3. 4264459927

3. 4692122376

4. 5489173285

2. 9740555135

$-1.8436071720$

$-2.8789975398$

$-2.1355106439$

1. 3553953501

2. 0321136211

0.5469410808

$-1.5329167484$

$-3.1349383363$

$-2.7911302462$
} 


\begin{abstract}
2.9529867496
5.1464095708

5.3892892284

3.4083157291

1. 2169310257
\end{abstract}

\section{$\mathrm{C}_{\mathrm{v}, \mathrm{c} 1}$}

Geometry:

C $\quad 0.2930271036$

C $\quad 0.3195603541$

P $\quad 1.8281697420$

C $\quad 1.3610613322$

C 2.2608786407

$\mathrm{Pd} \quad 3.9009963490$

P $\quad 4.8104959603$

C $\quad 6.5883140083$

C $\quad 7.3649683129$

$\mathrm{Cl} \quad 5.6071538386$

C $\quad 4.0844142808$

C $\quad 3.2664203816$

C $\quad 4.7516918010$

4.1005854471

1.5114449061

0.7087896958

$-0.6653820211$

$-0.5932660264$

1. 2638124288

0.0939091775

0.6539152931

2.1254627412

2. 9929805479

2.8224551915

4. 3121463621

3.5674404130

4.0782509335

5.2784389416

6.9956483693

8.4147096667

6.9759720270

0.3311808675

1. 9913441777

3.2906706248

2. 2156310194

2. 9357735580

4. 0061135324

4.3384999463

3. 6053698082

2.5338675338

2.6765901504

4. 5688874453

5.1709412966

3. 8617387036

1. 9543842901

$\mathrm{Cl} \quad 0.8562755833$

\section{$\mathrm{TS}_{\mathrm{V}, \mathrm{Cl}}{ }^{\mathrm{ox}}$}

Geometry:

C $\quad 0.0018406440$

$\mathrm{Cl} \quad 0.0749552600$

Pd $\quad 2.0596799077$

C $\quad-0.7784525290$

C $\quad-0.1210579217$

-0.1210579217
-0.8886500616

$-1.6079653727$

$-1.5554901506$

6.7290091946

4.1663137196

5.1447029661

4.7102073364

2.7334868264

4. 4931128997

5.0063019217

1.9080391690
$-1.1996997956$

$-1.1194238415$

0.0380729108

1.1419501461

1.0858774363

1 Total energy: $-0.0273157546$

$-0.0163456108$

$-0.1029419447$

1. 1014005884

1. 9508262198

0.0303633316

$-0.9245430260$

$-0.6545562503$

0.1197958010

1. 6010846494

$-0.4226804424$

$-1.1381848579$

$-2.7594051141$

$-3.5402594514$

$-1.6516822472$

$-2.6684034146$

0.0151061736

0.0396582420

$-0.0605081179$

$-2.6354649201$

$-3.5790499182$

$-1.7358571404$

$-2.1631983577$

$-0.7139755140$

0.6098637327

$-3.1088034718$

$-4.6250280624$

$-3.2084181181$

$-1.1381386623$

0.2852140998

0.6104948000

1.0989856454

2. 6473696411

1.9737573976

4. 9700291845

3. 9799705520

4. 3562026088

5.7078659168

6.6869705677

6.3232053121

2.9295685753

3. 5832543592

5. 9932059448

7.7391552659

7.0723926218

4.5120097530

17 Total energy:

0.0005033184

0.0134293291

$-0.0046835296$

$-1.0305117217$

1. 3170062081

1. 5597641034

0.5291940827

$-0.7605070028$

$-1.6862610755$

1.2111508237

0.6272799261

$-0.3371169809$

$-2.1096748177$

$-2.5933257523$

$-3.4249962126$

$-3.6182147418$
0.4976397281

$-0.6322254048$

$-2.8250213598$

3.8578813580

$-2.7188155743$

$-2429.58013108883$

0.1241207256

1. 4599690631

$-0.8920322228$

$-2.2118102470$

$-2.7197697812$

0.1272236770

2. 0291824679

2. 4533485208

1. 6877058719

$-1.1994174728$

3. 6464821300

4.4304214929

2.2035236181

1. 3364628226

$-1.8380316800$

$-1.4951854476$

$-0.4007434317$

2.0528661835

1.9995834507

$-0.5983469175$

$-2.0905408218$

$-2.7366398061$

4.1891460749

5. 3300148548

3. 9188597227

0.4911962748

1. 4435454744

3. 0500916922

3. 3468134389

1. 9357997225

0.7934486827

$-2.5820886799$

$-3.5151950686$

$-2.3588061092$

1. 9308818218

1. 2622974431

0.4436868150

0.3071391248

0.9834773694

1. 8033433299

1. 3634744144

$-0.0844238533$

$-0.3314148703$

0.8770561151

2. 3344535621

2. 9732025321

$-2429.53690619290$

0.0325330483

2. 1765331410

0.3740167679

$-0.5309841543$

$-0.4580375429$

$-1.6063828610$

$-2.2091255383$

$-1.6532896535$

$-3.1223833392$

0.8201877161

2. 2536113355

3. 0688040489

$-0.6582094607$

$-0.5921559334$

0.3218921818

$-0.0091045340$ 


\begin{abstract}
1. 2153678456
2.3219527069

3.1851013715

5.4244162779

5.2171079876

3. 9783627385

4.1001278027

2.0499897102

0.7729433479

1. 0602402950

4. 4058950236

6.0729140497

5.1652981963

4.3668944547

3. 9245507688

3. 7016714634

3. 7430687013

5.3086084823

6.1230397284

6.3913570355

5.9891927926

4.2688110976

1. 2509565182

2. 8038800896

4.2731359326

0.3639812763

$-0.9444372735$

$-2.2199877857$

$-2.1175384829$

$-0.7257021280$
\end{abstract}

\section{$I I_{\mathrm{v}, \mathrm{Cl}}$}

Geometry:

$\mathrm{Pd} \quad 1.7143625042$

C $\quad-0.1062146511$

$\mathrm{Cl} \quad 0.4505075797$

C $\quad-0.8350354537$

$-0.6914688084$

$-1.9715411482$

$-2.6860014125$

$-2.1128910472$

4. 9247507297

3.8143552290

5.2143010328

5.0786974469

2. 6733971857

4.4058386883

5.4217074519

2. 6391533202

2.2577429508

1. 7976168210

2. 2051295791

4. 6232295801

4.1141649703

3. 5863618532

4. 1862522317

2. 9651498195

2. 2558997705

1. 9251320823

5. 3020558976

6.4325984349

4. 5905131500

4. 8922287708

3. 9686164501

2. 8530927103

4.1129977974

5. 9322448132

6.2022824864

5. 5667477287

4. 6140526205

3.1831429982

0.8418171974

1. 5780587077

3.1190707310

$-0.1468338372$

$-2.4056233979$
$-3.6399869178$

$-2.1915292003$

$-2.2306953101$

1. 2979174715

0.8305542508

2.9964228524

3. 5771378734

$-4.5348239583$

$-4.5605088463$

$-2.7374178042$

$-3.9118141274$

$-3.6409482866$

$-2.1445724410$

3. 0086595366

4.6427743709

3. 6008312194

$-0.8104559401$

$-0.6925958324$

1.0845063937

1. 7272216947

0.8207548571

0.3794215222

$-2.0984043233$

$-2.1905295846$

$-2.2616542437$

2. 1366579510

2. 5721881401

0.7235210428

$-1.5716168977$

$-2.0324566711$

8 Total energy:

0.0074017011

0.9497002728

$-2.0722107948$

1. 1698120665

1. 3073792927

1.8721252032

2.0971634145

1. 7410690459

2.2732500673

$-1.3153126333$

$-0.9936148693$

$-0.4175307645$

2. 1502417979

2.3228944287

2. 5714120480

2. 9810203970

2.3809082090

3.3556521012

3. 7698416168

$-1.2388570778$

$-0.5534586515$

$-3.1224934093$

$-3.9007221558$

4.0219048603

2. 9044238148

1. 3455247041

2. 6768243797

2.6666955786

2. 2179570994

$-3.5089170778$

$-4.9658670780$

$-3.5379843187$

$-0.0716720737$

$-0.2516246428$

$-1.3097325701$

$-1.7765803450$

$-0.4795307098$

$-0.0007453677$

3.6789136215

4. 4638512417

3. 4304165661

1. 1548252550

2.1400599076
1. 1344537557

$-2.4365579451$

$-3.4609108521$

$-0.4949865160$

$-1.7322012996$

1.2107576302

2.4099430239

$-0.5845449887$

1. 5148157063

1. 7230742316

1.0884713132

0.3175631564

$-1.3375140118$

3.2977848666

2.5468309906

0.3449636414

2. 9143985302

3. 9064360733

2. 4149750607

$-0.2260044998$

$-2.4952738068$

$-2.0147777401$

$-2.6246667484$

$-4.4822447900$

$-3.3564178788$

0.0649031215

$-2.0010965147$

$-3.0860241180$

$-2.1127081522$

$-0.1209591148$

$-2429.58944124897$

$-0.0240323724$

$-0.0007712880$

$-0.1423199296$

$-1.1768334001$

1. 2218576188

1. 2682733748

0.0884270931

$-1.1350679700$

3.9966995701

0.0886256990

$-1.0521857604$

$-2.2491266248$

0.0672363543

0.6010247154

$-0.2311823270$

$-1.5649704338$

$-2.6954382338$

1. 1214831670

2. 3273338307

1. 7172018402

2. 7483949022

$-0.1225315579$

$-1.0270474979$

$-1.5755550655$

$-3.6504467941$

$-2.6966226174$

$-1.3077614974$

0.1613517347

1. 6826750864

$-1.7550366472$

$-1.0832226203$

0.5645478368

$-2.6091268840$

$-2.9048000781$

$-0.7132600070$

1.8237838270

3.7080724900

2.6633514821

0.7130315134

2.8879599926

2.8251800773

2.1501777849

2. 2301502207 
$1_{\mathrm{Ph}}$ Geometry: 69 Pd -0.0215295546 -0.0031451287 $\begin{array}{lr}\mathrm{P} & -0.0031451287 \\ \mathrm{P} & 0.0080889589\end{array}$ C $\quad-2.5940987030$ $-1.1873599126$ $-1.3493776775$ $-3.6597092960$ $-3.4904377553$ $-2.2524241835$

2. 1049613503

2.1888752008

1. 5432671958

3. 2814659825

3. 9066614931

3. 3566645497

$-0.3487519749$

0.4042707515

0.3233710453 $-0.9365766987$

$-0.8464339038$ $-0.1695158076$

1. 6289250413

0.8993749058

$-1.4689084012$

$-1.1831143988$

$-1.7257390685$

$-1.0475993773$

$-1.9604285387$

$-2.6289926044$

$-2.5134818056$

$-0.0091666664$

$-1.5995757098$

$-0.5627142169$

$-0.4904714077$

$-1.5321751180$

$-2.0876909498$

4.2946623635

2. 7833508757

4.0788552139

3. 2084515920

1. 9083959317

1. 6816190042

$-2.7115549875$

$-0.2279294789$

$-4.6165290077$

$-4.3161516356$

$-2.1118885710$

1.7737633143

3.7092061158

4. 8222780001

3. 8431881655

$-0.4223193440$

0.7729820194

$-1.3103288554$

$-0.6858347192$

$-1.6455651247$

$-2.0545163079$

$-3.2438751559$

$-3.0380991438$

0.8025165739

$-2.0071154418$

$-0.0486617956$

$-1.9034142729$

$-2.8909083331$

5.3044269067

2. 6139337447

4.9195256660

3. 3700974394

1.0712140027 53

$0.0066750479-0.0015757180$

$0.0046453463 \quad 2.3317309318$

$-0.0127724922-2.3349494653$

2.4680661916

4.3093470895

3.1210045076

3.0040846791

4.1908840486

4.8381980156

4.2800393141

2.3969134460

3.0843618302

4.7818258233

4.0998058453

2. 9056418815

4.1871758269

2.4452645983

2.9802018365

4.8558314459

4. 3275971318

3.1191621240

4.8164053957

1.4914929465

5.7898247090

$-2.4413825053$

$-4.3389576041$

$-3.1229783714$

$-2.9755096750$

$-4.1895878097$

$-4.8664039353$

$-4.3127457496$

$-2.4971749446$

$-3.1331524548$

$-4.8489336679$

$-4.2183134474$

$-3.0412243329$

$-4.0453771151$

$-2.3923057187$

$-2.8812792566$

$-4.7165592208$

$-4.2353670115$

$-3.0715347054$

1. 5288853273

4.8185770188

2.4881994157

4.6034012356

5.7561926789

1.4527695290

5.7058684705

4. 4914805236

2.3628320486

4.5991376567

2. 4479830045

4.8534058570

$-1.4823946803$

$-4.8709875217$

$-2.4372198529$

$-4.6011604230$

$-5.8063457082$

$-4.8091857889$

$-1.5649372172$

$-5.7594454857$

$-4.6364877183$

$-2.5387657899$

$-4.4208642976$

$-1.4690185323$

$-2.3457300691$

$-5.6165549391$

$-4.7639002906$ 


\begin{tabular}{|c|c|c|c|}
\hline $\mathrm{Cl}$ & 0.78300 & -0.64964 & -0.05347 \\
\hline $\mathrm{C}$ & 1.98430 & -0.51522 & 5.57147 \\
\hline C & 3.70942 & 0.93685 & 4.71328 \\
\hline $\mathrm{C}$ & 2.36316 & 0.54457 & 4.72502 \\
\hline C & 2.91581 & -1.14317 & 6.39750 \\
\hline C & 4.25583 & -0.74437 & 6.37619 \\
\hline C & 4.64796 & 0.29256 & 5.52703 \\
\hline C & 2.34955 & 3.82379 & 3.28137 \\
\hline C & 2.27521 & 2.35959 & 1.35200 \\
\hline $\mathrm{C}$ & 1.97357 & 2.59911 & 2.70265 \\
\hline $\mathrm{C}$ & 3.02832 & 4.78647 & 2.53049 \\
\hline C & 3.33794 & 4.53681 & 1.18914 \\
\hline C & 2.95967 & 3.32560 & 0.60572 \\
\hline C & -1.55809 & 3.89962 & 6.46772 \\
\hline C & -1.11233 & 2.88108 & 4.31391 \\
\hline C & -1.91525 & 3.67738 & 5.13176 \\
\hline C & -0.38931 & 3.32315 & 6.96980 \\
\hline C & 0.42507 & 2.53973 & 6.14299 \\
\hline C & 0.07687 & 2.30645 & 4.80272 \\
\hline $\mathrm{P}$ & 1.05407 & 1.25616 & 3.61277 \\
\hline $\mathrm{H}$ & 2.10704 & 4.02660 & 4.32145 \\
\hline $\mathrm{H}$ & -1.41149 & 2.68071 & 3.28763 \\
\hline $\mathrm{H}$ & -0.10348 & 3.48456 & 8.00756 \\
\hline C & -3.57699 & 0.18817 & 3.90420 \\
\hline C & -4.62031 & -1.31007 & 2.32607 \\
\hline C & -3.49125 & -0.94760 & 3.07618 \\
\hline C & -4.76058 & 0.91954 & 3.99968 \\
\hline C & -5.88027 & 0.54868 & 3.24603 \\
\hline C & -5.80187 & -0.56376 & 2.40586 \\
\hline C & -3.00061 & -4.35810 & 2.28187 \\
\hline C & -1.65347 & -3.26815 & 0.58742 \\
\hline C & -2.21932 & -3.26276 & 1.87256 \\
\hline C & -3.22677 & -5.43260 & 1.41920 \\
\hline C & -2.66991 & -5.42699 & 0.13528 \\
\hline C & -1.88491 & -4.34687 & -0.27434 \\
\hline $\mathrm{C}$ & -1.36774 & -3.96946 & 7.08209 \\
\hline C & -0.50285 & -3.25118 & 4.93461 \\
\hline C & -0.32341 & -3.91178 & 6.15089 \\
\hline C & -2.59010 & -3.36636 & 6.77877 \\
\hline C & -2.77436 & -2.71903 & 5.55101 \\
\hline C & -1.73425 & -2.65048 & 4.61030 \\
\hline $\mathrm{P}$ & -1.84167 & -1.79357 & 2.95809 \\
\hline $\mathrm{Pd}$ & -0.09683 & -0.36410 & 2.41113 \\
\hline $\mathrm{H}$ & 0.95324 & -0.85542 & 5.56412 \\
\hline $\mathrm{H}$ & 4.02823 & 1.74496 & 4.06248 \\
\hline $\mathrm{H}$ & 2.59505 & -1.95602 & 7.04540 \\
\hline $\mathrm{H}$ & 4.98661 & -1.24377 & 7.00894 \\
\hline $\mathrm{H}$ & 5.68859 & 0.60609 & 5.49537 \\
\hline $\mathrm{H}$ & 1.94880 & 1.43050 & 0.88201 \\
\hline $\mathrm{H}$ & 3.31175 & 5.73135 & 2.99069 \\
\hline $\mathrm{H}$ & 3.86347 & 5.28826 & 0.60245 \\
\hline $\mathrm{H}$ & 3.18344 & 3.12827 & -0.44003 \\
\hline $\mathrm{H}$ & -2.18971 & 4.50906 & 7.11058 \\
\hline $\mathrm{H}$ & -2.82607 & 4.11550 & 4.72899 \\
\hline $\mathrm{H}$ & 1.33473 & 2.10426 & 6.54609 \\
\hline $\mathrm{H}$ & -2.69994 & 0.50522 & 4.46127 \\
\hline $\mathrm{H}$ & -4.57711 & -2.17587 & 1.67298 \\
\hline $\mathrm{H}$ & -4.80053 & 1.78968 & 4.65114 \\
\hline $\mathrm{H}$ & -6.79964 & 1.12753 & 3.30760 \\
\hline $\mathrm{H}$ & -6.66248 & -0.85725 & 1.80836 \\
\hline $\mathrm{H}$ & -3.42891 & -4.37249 & 3.28085 \\
\hline H & -1.01102 & -2.44277 & 0.27636 \\
\hline H & -3.83216 & -6.27469 & 1.74963 \\
\hline $\mathrm{H}$ & -2.84163 & -6.26636 & -0.53612 \\
\hline $\mathrm{H}$ & -1.43629 & -4.34082 & -1.26495 \\
\hline $\mathrm{H}$ & -1.22547 & -4.47257 & 8.03632 \\
\hline $\mathrm{H}$ & 0.32086 & -3.17793 & 4.22803 \\
\hline $\mathrm{H}$ & 0.63581 & -4.37285 & 6.37617 \\
\hline $\mathrm{H}$ & -3.40810 & -3.40008 & 7.49606 \\
\hline $\mathrm{H}$ & -3.73253 & -2.25901 & 5.32854 \\
\hline
\end{tabular}

$\mathrm{C}_{\mathrm{ph}}$ 


\begin{tabular}{|c|c|c|c|}
\hline $\mathrm{Geo}$ & Total & energy & \\
\hline C & 0.0541078675 & -0.0495747860 & -0.0513516536 \\
\hline C & 0.0814420327 & 0.0353673680 & 1.3509753925 \\
\hline $\mathrm{C}$ & 1.2941875939 & 0.0994782359 & 2.0367555796 \\
\hline C & 2.5010739143 & 0.0596456417 & 1.3345870652 \\
\hline $\mathrm{C}$ & 2.4847045289 & -0.0403394440 & -0.0569763576 \\
\hline C & 1.2702494664 & -0.0910102661 & -0.7475071524 \\
\hline $\mathrm{P}$ & -1.6024380149 & -0.1012466717 & -0.8780409398 \\
\hline C & -2.0291760039 & 1.6878512125 & -1.0634187785 \\
\hline $\mathrm{C}$ & -1.0965700482 & 2.7269279420 & -0.9226548544 \\
\hline $\mathrm{C}$ & -1.4921597184 & 4.0576370822 & -1.0775686286 \\
\hline C & -2.8192958257 & 4.3662045183 & -1.3781665486 \\
\hline C & -3.7552714217 & 3.3389992746 & -1.51859 \\
\hline C & -3.3646420140 & 2.0109013558 & -1.3534 \\
\hline $\mathrm{Pd}$ & -3.1773211330 & -1.4005806731 & 0.2490080432 \\
\hline $\mathrm{P}$ & -4.6009027478 & -2.6169314692 & 1.6269166278 \\
\hline C & -6.4150985638 & -2.3910101288 & 1625846 \\
\hline C & -6.8708066420 & -1.0801445359 & 81574 \\
\hline C & -8.2226923371 & -0.8226318470 & 7294 \\
\hline C & -9.1419955365 & -1.8747707917 & 2806 \\
\hline $\mathrm{C}$ & -8.6989725474 & -3.1802659125 & 9472 \\
\hline C & -7.3456876677 & -3.4392367576 & 1.32 \\
\hline C & -4.3712084069 & -4.4513957146 & 1.62 \\
\hline $\mathrm{C}$ & -3.9381344721 & -5.0541322196 & 0.42 \\
\hline $\mathrm{C}$ & -3.7542398303 & -6.4349177177 & 0.3 \\
\hline $\mathrm{C}$ & -3.9854432131 & -7.2332721226 & 9040 \\
\hline C & -4.4024679957 & -6.6424949454 & $2.6^{\prime}$ \\
\hline $\mathrm{C}$ & -4.5960994698 & -5.2620134360 & 2.7 \\
\hline C & -4.4170420448 & -2.1784201732 & 3.4 \\
\hline C & -5.5012975367 & -2.0390681898 & 38066 \\
\hline $\mathrm{C}$ & -5.2895622299 & -1.6842897324 & 2410 \\
\hline $\mathrm{C}$ & -3.9946085854 & -1.4723390859 & $\$ 345$ \\
\hline C & -2.9079403437 & -1.6109355804 & 8471 \\
\hline C & -3.1178558856 & -1.9525814929 & 1437 \\
\hline C & -1.1917205423 & -0.6169763724 & -2.60 \\
\hline C & -1.1798790789 & -1.9925072476 & -2.8 \\
\hline $\mathrm{C}$ & -0.8687293745 & -2.4527742802 & -4.1 \\
\hline C & 826519671 & -1.5437014240 & -5.1 \\
\hline C & -0.6059709692 & -0.1745223985 & -4.9 \\
\hline C & -0.9053058907 & 0.2878928049 & -3.6 \\
\hline $\mathrm{H}$ & -6.5143170648 & -2.2000838668 & 3.9 \\
\hline $\mathrm{H}$ & -6.1551126309 & -0.2601112581 & 1.1 \\
\hline $\mathrm{H}$ & -9.4072221234 & -4.0051854063 & 3112 \\
\hline $\mathrm{H}$ & -3.7328562237 & -4.4277357816 & -0.435 \\
\hline $\mathrm{H}$ & -4.9161598381 & -4.8140232864 & 712 \\
\hline $\mathrm{H}$ & -3.4189147279 & -6.8855169887 & -0.57 \\
\hline $\mathrm{H}$ & -3.8331354076 & -8.3081456132 & 1.422 \\
\hline $\mathrm{H}$ & -4.5756546284 & -7.2565784563 & 7447 \\
\hline $\mathrm{H}$ & -2.2740484957 & -2.0343104562 & 2901 \\
\hline $\mathrm{H}$ & -6.1405356717 & -1.5722114589 & 6.295 \\
\hline $\mathrm{H}$ & -3.8323412281 & -1.1934291322 & 7.139 \\
\hline $\mathrm{H}$ & -1.8963196890 & -1.4407874033 & 6644 \\
\hline $\mathrm{H}$ & -10.1943954222 & -1.6772696748 & 0.695 \\
\hline $\mathrm{H}$ & -8.5537998544 & 0.1966731965 & 0.722 \\
\hline $\mathrm{H}$ & -7.0172417964 & -4.4609100883 & 4818 \\
\hline $\mathrm{H}$ & -1.4261413378 & -2.6979497016 & -2.0971 \\
\hline $\mathrm{H}$ & -0.9214564282 & 1.3561489879 & -3.4 \\
\hline $\mathrm{H}$ & -0.8589326457 & -3.5210984836 & -4.3 \\
\hline $\mathrm{H}$ & -0.3495963224 & -1.9013357113 & -6.1876 \\
\hline $\mathrm{H}$ & -0.3915885702 & 0.5385210712 & -5.7135 \\
\hline $\mathrm{H}$ & -0.0603083187 & 2.4997212512 & -0.6899 \\
\hline $\mathrm{H}$ & -4.0982944417 & 1.2153783076 & -1.4528 \\
\hline $\mathrm{H}$ & -0.7596480823 & 4.8533502084 & -0.9623 \\
\hline $\mathrm{H}$ & -3.12474252 & 5.4030380205 & -1.497 \\
\hline $\mathrm{H}$ & -4.7932503869 & 3.5689019547 & -1.7454248786 \\
\hline $\mathrm{H}$ & 3.4467787574 & 0.1005074393 & 1.8696609216 \\
\hline $\mathrm{H}$ & -0.8565974761 & 0.0455113952 & 1.9005271793 \\
\hline $\mathrm{H}$ & 1.2973894054 & 0.1720397914 & 3.1215608386 \\
\hline $\mathrm{H}$ & 3.4189963456 & -0.0774672538 & -0.6112246859 \\
\hline $\mathrm{H}$ & 1.2727296352 & -0.1632006259 & -1.8310074659 \\
\hline $\mathrm{Cl}$ & -7.7098126599 & 1.9064184564 & -2.0202995602 \\
\hline $\mathrm{C}$ & -7.0768856613 & 0.6735364048 & -3.1168528158 \\
\hline $\mathrm{C}$ & -7.3159251702 & 0.7935213177 & -4.4840340286 \\
\hline C & -6.8045078620 & -0.1782755541 & -5.3459334669 \\
\hline $\mathrm{C}$ & -6.0664858416 & -1.2504010141 & -4.8443429096 \\
\hline C & -5.8399043292 & -1.3527760544 & -3.4721665536 \\
\hline $\mathrm{C}$ & -6.3464528876 & -0.3909321755 & -2.595754259 \\
\hline
\end{tabular}


-7.8866436841
-6.9836622124
-5.6674278045
-5.2608990289

$-6.1722342712$
1.6329402217

$-0.0908034232$ $-2.0007254384$

$-2.1780461044$

$-0.4711524223$
$-4.8652299298$

$-6.4141001175$

$-5.5211635346$

$-3.0691270959$

$-1.5291307815$

$\mathrm{C}_{\mathrm{Ph}, \mathrm{Cl}}$

Geometry: 10 Total energy: -3351.50617078550

$-0.1655117542$

1. 0233613125

2. 2519313991

2. 2804391688

1.0885027773

$-1.7973471334$

$-3.6545673466$

$-4.1333572201$

$-2.7144037103$

$-1.7654068431$

$-0.6577214147$

$-0.4597009456$

$-1.3851712097$

$-2.5072144519$

$-4.8811310014$

$-4.5304624024$

$-5.0513582198$

$-5.9471181021$

$-6.3181271575$

$-5.7887147892$

$-5.3582396076$

$-5.2315117040$

$-6.1900284141$

$-7.2893714852$

$-7.4207012689$

$-6.4597860519$

$-1.3128034335$

$-1.0835197789$

$-0.7641629426$

$-0.6855961568$

$-0.9239244673$

$-1.2312392420$

$-1.9735227188$

$-0.9136373677$

$-1.1407091851$

$-2.4303096125$

$-3.4913935384$

$-3.2720092552$

$-0.1288556287$

0.0490334297

1149670083

1.4521112863

$-0.1446861662 \quad 2.1828320975$

$-0.2110511854 \quad 1.5215762206$

$-0.2445611625 \quad 0.1255633504$

$-0.1993988467-0.6044774167$

$-0.0743268066-0.8294381059$

$\begin{array}{ll}-0.9555333552 & 0.2328376786\end{array}$

$-2.0598984958 \quad 2.2167007869$

$-2.8462324531 \quad 3.1361497016$

$-3.5425675545 \quad 2.3657695628$

$\begin{array}{ll}-4.1439449934 & 2.9639864358\end{array}$

$-4.0405964540 \quad 4.3468669093$

$-3.3394097557 \quad 5.1218335889$

$-2.7539222626 \quad 4.5233186349$

$-0.9678364899 \quad 3.5200485972$

$0.3921544883 \quad 3.4962998465$

$1.2822219558 \quad 4.4381152386$

$0.8289363848 \quad 5.4109631013$

$-0.5188073190 \quad 5.4332750879$

$-1.4108837265 \quad 4.4952111587$

$-3.4526119638 \quad 2.1325654740$

$\begin{array}{ll}-4.6690503944 & 2.8232632468\end{array}$

$\begin{array}{ll}-5.6759327446 & 2.6713607902\end{array}$

$-5.4760125261 \quad 1.8331918269$

$-4.2670977555 \quad 1.1409285222$

$-3.2626099809 \quad 1.2762693910$

$-0.7541537832 \quad-2.4911984914$

$-2.1397902057 \quad-2.5856211148$

$-2.7336666156-3.8061225317$

$-1.9563225028 \quad-4.9661966088$

$-0.5824314862 \quad-4.8891773140$

$0.0150146696-3.6618071282$

$1.7478394170-1.1642592776$

$2.6689891174-1.1185522070$

$4.0267031538-1.3553449254$

$4.4808357579 \quad-1.6471307185$

$3.5705418956-1.6890560261$

$2.2137152193-1.4420346415$

$-0.3696935876-1.0252059451$

$-0.1096454994 \quad 1.9599310300$

$-0.1383569215 \quad 3.2691184356$

$-0.2497541341 \quad 2.0899308328$

$-0.3064510471-0.3988794533$

$-0.2246353702 \quad-1.6897297294$

$-3.5947703861 \quad 1.2874519173$

$\begin{array}{ll}-4.6788002919 & 2.3466097737\end{array}$

$-4.4948595561 \quad 4.8122371622$

$-3.2469497231 \quad 6.1972025128$

$-2.2180440616 \quad 5.1413264518$

$0.7442195015 \quad 2.7140035600$

2.3319221628 4.4010265127

$1.5230315318 \quad 6.1389819060$

$-0.8780778448 \quad 6.1796713353$

$-2.4561593852 \quad 4.5171499876$

$\begin{array}{ll}-4.8339900851 & 3.4756879390\end{array}$

$\begin{array}{ll}-6.6166757237 & 3.2064882528\end{array}$

$-6.2614095197 \quad 1.7104608734$

$\begin{array}{ll}-4.1088040558 & 0.4726560580\end{array}$

$-2.3402242364 \quad 0.6992987238$

$-2.7579437444 \quad-1.6963299445$

$-3.8067620039-3.8524242662$

$-2.4205819284 \quad-5.9211826886$

$0.0313965277 \quad-5.7855357130$

$1.0843900318-3.6158831462$

$2.3273664402 \quad-0.8927669182$

$4.7279938761-1.3114461981$

$5.5385444543-1.8295631590$ 


$$
\begin{aligned}
& -4.5011004672 \\
& -4.1080885691 \\
& -5.2001242724 \\
& -4.1338687126 \\
& -3.6245578550 \\
& -4.1507415193 \\
& -5.2171574362 \\
& -5.7389287226 \\
& -5.6057844784 \\
& -3.7033108604 \\
& -2.2618980157 \\
& -3.7521152746 \\
& -5.6209201364 \\
& -6.5681648274
\end{aligned}
$$

$-1.9025150417$

$-1.4461872791$

$-3.7313837742$

$-2.9303550438$

\begin{tabular}{|c|c|c|c|}
\hline $\mathrm{Cl}$ & 0.0211052703 & -0.1152647680 & -0.0724948051 \\
\hline C & -0.0144064145 & 0.0282145650 & 1.9502519918 \\
\hline $\mathrm{Pd}$ & 2.1164574893 & -0.0586847229 & 1.3093974575 \\
\hline C & -0.5679300895 & -1.0881485069 & 2.6052113408 \\
\hline C & -1.3758260020 & -0.8986292021 & 3.7234720014 \\
\hline $\mathrm{C}$ & -1.6531201371 & 0.3866379725 & 4.2044761395 \\
\hline C & -1.1022481376 & 1.4911586293 & 3.5490509957 \\
\hline C & -0.3035356541 & 1.3257456407 & 2.4162166460 \\
\hline $\mathrm{H}$ & -0.3841153673 & -2.0863534362 & 2.2201068497 \\
\hline $\mathrm{H}$ & -1.7945460188 & -1.7692305806 & 4.2235971571 \\
\hline $\mathrm{H}$ & -2.2973165860 & 0.5239523728 & 5.0690658107 \\
\hline $\mathrm{H}$ & -1.3054031750 & 2.4975530646 & 3.9100254370 \\
\hline $\mathrm{H}$ & 0.0753834588 & 2.1905855930 & 1.8808087042 \\
\hline $\mathrm{P}$ & 3.3382445144 & -0.4639933585 & 3.3540558024 \\
\hline C & 4.8823938971 & -1.4505998798 & 3.0704973905 \\
\hline C & 3.9269496914 & 1.0951490552 & 4.1649236927 \\
\hline $\mathrm{C}$ & 2.6044791080 & -1.3474890481 & 4.80719 \\
\hline C & 6.0645863557 & -1.2938933287 & 3.8104523436 \\
\hline C & 7.1675218465 & -2.1148312680 & 3.56899 \\
\hline C & 7.1058217812 & -3.1101743165 & 2.59124 \\
\hline C & 5.9356519431 & -3.2737799895 & 1.8482959308 \\
\hline $\mathrm{C}$ & 4.8366901294 & -2.4452664787 & 2.0810 \\
\hline $\mathrm{H}$ & 6.1310142655 & -0.5262583227 & 4.5753 \\
\hline $\mathrm{H}$ & 8.0768928214 & -1.9767963733 & 4.14947 \\
\hline $\mathrm{H}$ & 7.9657242831 & -3.7486646495 & 2.40 \\
\hline $\mathrm{H}$ & 5.8796116430 & -4.0393374510 & 1.07 \\
\hline $\mathrm{H}$ & 3.9329249126 & -2.5615586192 & 1.4868539497 \\
\hline C & 4.3634787505 & 1.1413362650 & 5.5010131579 \\
\hline C & 4.8044396695 & 2.3385900323 & 6.0640 \\
\hline C & 4.8103518877 & 3.5116023553 & 5.30565 \\
\hline C & 4.3673098586 & 3.4818139214 & 3.9835738701 \\
\hline $\mathrm{C}$ & 3.9240973649 & 2.2834609481 & 3.41 \\
\hline $\mathrm{H}$ & 4.3438346294 & 0.2415003091 & 6633 \\
\hline $\mathrm{H}$ & 5.1385375770 & 2.3560840334 & $\$ 8685$ \\
\hline $\mathrm{H}$ & 5.1509388240 & 4.4446279097 & 5.74807 \\
\hline $\mathrm{H}$ & 4.3561083961 & 4.3908824834 & 3.3877 \\
\hline $\mathrm{H}$ & 3.5586899354 & 2.2709000210 & 2.3962314852 \\
\hline $\mathrm{C}$ & 3.0184858750 & -2.6250465203 & 5.21253 \\
\hline C & 2.4181791767 & -3.2519647202 & 6.30 \\
\hline $\mathrm{C}$ & 1.4000418898 & -2.6123556046 & 7.0129195137 \\
\hline $\mathrm{C}$ & 0.9735720178 & -1.3452535098 & 6.6093054844 \\
\hline C & 1.5624135567 & -0.7217619313 & 5.51111 \\
\hline $\mathrm{H}$ & 3.8209907906 & -3.1312949550 & 4.6855748513 \\
\hline $\mathrm{H}$ & 2.7571239926 & -4.2394503231 & 6.6108409785 \\
\hline $\mathrm{H}$ & 0.9390394198 & -3.0981426718 & 7.8693469916 \\
\hline $\mathrm{H}$ & 0.1714232037 & -0.8417937366 & 7.1431771675 \\
\hline $\mathrm{H}$ & 1.2059722828 & 0.2565997922 & 5.2015363842 \\
\hline $\mathrm{P}$ & 3.7642169105 & 0.6908589424 & -0.5024902421 \\
\hline $\mathrm{C}$ & 5.5831013397 & 0.3826682099 & -0.6093538657 \\
\hline $\mathrm{C}$ & 3.1411044568 & 0.2040039106 & -2.1716809370 \\
\hline $\mathrm{C}$ & 3.6472955407 & 2.5401688232 & -0.5601013706 \\
\hline $\mathrm{C}$ & 6.2222260196 & -0.0735876226 & -1.7714363024 \\
\hline $\mathrm{C}$ & 7.5969588942 & -0.3187770182 & -1.7740894233 \\
\hline $\mathrm{C}$ & 8.3531551834 & -0.1083404929 & -0.6213446688 \\
\hline C & 7.7268923854 & 0.3440267725 & 0.5417616966 \\
\hline $\mathrm{C}$ & 6.3530365882 & 0.5784798758 & 0.5502092166 \\
\hline $\mathrm{H}$ & 5.6487595591 & -0.2350652740 & -2.678563384 \\
\hline $\mathrm{H}$ & 8.0766041304 & -0.6727813183 & -2.683504991 \\
\hline $\mathrm{H}$ & 9.4232871357 & -0.3003424626 & -0.626598 \\
\hline
\end{tabular}

$-1.9765520539$

$-1.8011139149$

$-2.6103312624$

$-3.5728456263$

$-4.4789330152$

$-3.0415574488$

$-0.9706687549$

$-1.0545283166$

$-2.4554860511$

$-4.1996640034$

\section{$\mathrm{TS}_{\mathrm{Ph}}{ }^{\mathrm{ox}}$}

81

Geometry: 6 Total energy: -2891.17429855954 


$\begin{array}{lrr}8.3041793715 & 0.5030487478 & 1.4486898183 \\ 5.8779249351 & 0.9191088160 & 1.4658859321 \\ 3.2617138096 & 1.0283958270 & -3.3026468442 \\ 2.7521755025 & 0.6198869455 & -4.5354405936 \\ 2.1166222040 & -0.6185227961 & -4.6576430472 \\ 1.9966332872 & -1.4466631587 & -3.5411352355 \\ 2.4986037681 & -1.0358726069 & -2.3054534486 \\ 3.7472222845 & 1.9964856425 & -3.2167307734 \\ 2.8495757950 & 1.2714983895 & -5.4000519323 \\ 1.7146737051 & -0.9336077618 & -5.6168994287 \\ 1.4935962568 & -2.4063502639 & -3.6242913142 \\ 2.3779509408 & -1.6738122502 & -1.4348299600 \\ 4.7590054933 & 3.3948570091 & -0.5492512210 \\ 4.5885136951 & 4.7823426204 & -0.5443095262 \\ 3.3085634572 & 5.3351121806 & -0.5555885599 \\ 2.1938230717 & 4.4916653624 & -0.5658213580 \\ 2.3595655782 & 3.1079902118 & -0.5640976222 \\ 5.7629308488 & 2.9825151280 & -0.5445213078 \\ 5.4625036494 & 5.4295158971 & -0.5359669184 \\ 3.1779565134 & 6.4142502179 & -0.5526609073 \\ 1.1911532235 & 4.9122184741 & -0.5722686629 \\ 1.4849228982 & 2.4617345376 & -0.5655874514 \\ & & \end{array}$

$\mathrm{TS}_{\mathrm{Ph}, \mathrm{Cl}}{ }^{\mathrm{ox}}$

Geometry: 9 Total energy: $\quad-3351.45468187797$

\begin{tabular}{|c|c|c|c|}
\hline $\mathrm{Cl}$ & -0.0046045739 & -0.0659042946 & -0.0055588553 \\
\hline $\mathrm{C}$ & 0.0023262842 & -0.0122514423 & 1.9957838603 \\
\hline $\mathrm{Pd}$ & 2.1590612622 & -0.0099391502 & 1.4250861437 \\
\hline $\mathrm{C}$ & -0.5821322003 & -1.1393871818 & 2.6063962007 \\
\hline $\mathrm{C}$ & -1.2771855524 & -0.9860698493 & 3.7998150311 \\
\hline C & -1.4392080745 & 0.2778563429 & 4.3850210488 \\
\hline C & -0.9011178863 & 1.3969798140 & 3.7470246983 \\
\hline C & -0.2138190962 & 1.2699745738 & 2.5382051447 \\
\hline $\mathrm{H}$ & -0.4681691790 & -2.1176151539 & 2.1506705350 \\
\hline $\mathrm{H}$ & -1.6902068798 & -1.8672810490 & 4.2857230407 \\
\hline $\mathrm{H}$ & -1.9921625829 & 0.3865569431 & 5.3143043635 \\
\hline $\mathrm{H}$ & -1.0317262432 & 2.3875189629 & 4.1786277459 \\
\hline $\mathrm{H}$ & 0.1588604830 & 2.1482098007 & 2.0214702234 \\
\hline $\mathrm{P}$ & 3.4011008828 & -1.1024572534 & 3.1891409485 \\
\hline $\mathrm{C}$ & 4.5614416788 & -2.2685138932 & 2.3473555314 \\
\hline C & 4.4036822753 & 0.0763025712 & 4.1957263959 \\
\hline C & 2.7152274458 & -2.1542851146 & 4.5583829305 \\
\hline $\mathrm{C}$ & 5.9565909714 & -2.2686993378 & 2.4773659505 \\
\hline $\mathrm{C}$ & 6.7312037047 & -3.1936322808 & 1.7700945602 \\
\hline $\mathrm{C}$ & 6.1289013039 & -4.1237778675 & 0.9262388317 \\
\hline C & 4.7367025970 & -4.1270840634 & 0.7814973524 \\
\hline $\mathrm{C}$ & 3.9613167244 & -3.2035701245 & 1.4797551788 \\
\hline $\mathrm{H}$ & 6.5065016539 & -1.5742263355 & 3.1084655683 \\
\hline $\mathrm{H}$ & 7.8081758089 & -3.1486948502 & 1.9115269813 \\
\hline $\mathrm{H}$ & 6.7351604032 & -4.8408067855 & 0.3759757354 \\
\hline $\mathrm{H}$ & 4.2541972019 & -4.8448100763 & 0.1204774255 \\
\hline $\mathrm{H}$ & 2.8806349344 & -3.1998171247 & 1.3483264265 \\
\hline $\mathrm{C}$ & 5.4582564846 & -0.3212530842 & 5.0319109034 \\
\hline C & 6.1803671506 & 0.6247236862 & 5.7585280882 \\
\hline $\mathrm{C}$ & 5.8199336617 & 1.9713350675 & 5.7063222039 \\
\hline $\mathrm{C}$ & 4.7510202616 & 2.3770604985 & 4.9041381479 \\
\hline $\mathrm{C}$ & 4.0570678012 & 1.4375513972 & 4.1413703983 \\
\hline $\mathrm{H}$ & 5.7610946633 & -1.3599823821 & 5.0957173374 \\
\hline $\mathrm{H}$ & 7.0483893483 & 0.2991314541 & 6.3223054231 \\
\hline $\mathrm{H}$ & 6.3847176997 & 2.7073660973 & 6.2744423540 \\
\hline $\mathrm{H}$ & 4.4705487785 & 3.4271569804 & 4.8528485698 \\
\hline $\mathrm{H}$ & 3.2468728036 & 1.7528669655 & 3.4875077139 \\
\hline C & 3.1768161111 & -3.4476584311 & 4.8447787075 \\
\hline C & 2.6574529364 & -4.1643182064 & 5.9251546253 \\
\hline $\mathrm{C}$ & 1.6714983347 & -3.6011445476 & 6.7354331110 \\
\hline $\mathrm{C}$ & 1.2018601682 & -2.3156988465 & 6.4557538023 \\
\hline $\mathrm{C}$ & 1.7148848689 & -1.6015568473 & 5.3740031608 \\
\hline $\mathrm{H}$ & 3.9544730028 & -3.8925068774 & 4.2321075463 \\
\hline $\mathrm{H}$ & 3.0354132430 & -5.1621677073 & 6.1375346895 \\
\hline $\mathrm{H}$ & 1.2727255285 & -4.1585208173 & 7.5802957961 \\
\hline $\mathrm{H}$ & 0.4320931367 & -1.8664930886 & 7.0795675718 \\
\hline $\mathrm{H}$ & 1.3385312624 & -0.6039330847 & 5.1650179984 \\
\hline $\mathrm{P}$ & 3.7058554524 & 1.1255081764 & -0.1901954427 \\
\hline C & 5.5424879852 & 0.9485645677 & -0.2452451468 \\
\hline C & 3.2083416621 & 0.5583513605 & -1.8793698939 \\
\hline $\mathrm{C}$ & 3.4465393047 & 2.9594760349 & -0.2661414264 \\
\hline
\end{tabular}




$\begin{array}{ll}\mathrm{C} & 6.2654085033 \\ \mathrm{C} & 7.6505412374 \\ \mathrm{C} & 8.3257690202 \\ \mathrm{C} & 7.6229540074 \\ \mathrm{C} & 6.2368197862 \\ \mathrm{H} & 5.7463632406 \\ \mathrm{H} & 8.2003244101 \\ \mathrm{H} & 9.4022398106 \\ \mathrm{H} & 8.1321393128 \\ \mathrm{H} & 5.6929825288 \\ \mathrm{C} & 2.6790929716 \\ \mathrm{C} & 2.2596963020 \\ \mathrm{C} & 2.3684312674 \\ \mathrm{C} & 2.8966031549 \\ \mathrm{C} & 3.3031907561 \\ \mathrm{H} & 2.5906106362 \\ \mathrm{H} & 1.8471559568 \\ \mathrm{H} & 2.0395679648 \\ \mathrm{H} & 2.9815378871 \\ \mathrm{H} & 3.6958024397 \\ \mathrm{C} & 4.4947303189 \\ \mathrm{C} & 4.2411616135 \\ \mathrm{C} & 2.9387144254 \\ \mathrm{C} & 1.8855986708 \\ \mathrm{C} & 2.1383535872 \\ \mathrm{H} & 5.5130892766 \\ \mathrm{H} & 5.0681996111 \\ \mathrm{H} & 2.7444457791 \\ \mathrm{H} & 0.8658707155 \\ \mathrm{H} & 1.3149535066 \\ \mathrm{Cl} & 8.8760763459 \\ \mathrm{H} & \end{array}$

\section{$I I_{\mathrm{Ph}}$}

$\begin{array}{lrrrr}\text { Geometry: } & \text { 8 Total } & \text { energy: } & \text {-2891.22055914454 } \\ \text { Cl } & 0.0395797647 & 0.0402129977 & 0.0364228639 \\ \mathrm{C} & 0.0422630893 & -0.0376697907 & 3.0560305175 \\ \mathrm{Pd} & 1.6607619457 & 0.0145005718 & 1.8250950535 \\ \mathrm{C} & -0.3087223338 & -1.2267425994 & 3.7049379031 \\ \mathrm{C} & -1.4781822277 & -1.2982920067 & 4.4737261590 \\ \mathrm{C} & -2.2995966638 & -0.1765206084 & 4.6091774032 \\ \mathrm{C} & -1.9501860097 & 1.0134043724 & 3.9629219272 \\ \mathrm{C} & -0.7885233682 & 1.0812431673 & 3.1832538751 \\ \mathrm{H} & 0.3194767228 & -2.1101059296 & 3.6157964056 \\ \mathrm{H} & -1.7405079179 & -2.2320937995 & 4.9667165282 \\ \mathrm{H} & -3.2056490215 & -0.2296686107 & 5.2085308802 \\ \mathrm{H} & -2.5893502702 & 1.8897319619 & 4.0499544049 \\ \mathrm{H} & -0.5521415504 & 1.9994502452 & 2.6528237522 \\ \mathrm{P} & 3.0665199003 & 0.1531166093 & 3.7016114239 \\ \mathrm{C} & 4.3246428852 & -1.1966074770 & 3.7228574555 \\ \mathrm{C} & 3.9646654694 & 1.7632104027 & 3.7824487917 \\ \mathrm{C} & 2.3629772590 & 0.0718922168 & 5.4101443593 \\ \mathrm{C} & 5.6733588963 & -1.0130659276 & 4.0580707210 \\ \mathrm{C} & 6.5386537044 & -2.1093648212 & 4.1231627041 \\ \mathrm{C} & 6.0673101689 & -3.3976345740 & 3.8600955490 \\ \mathrm{C} & 4.7264827108 & -3.5877104871 & 3.5100027467 \\ \mathrm{C} & 3.8641786019 & -2.4925291261 & 3.4307467499 \\ \mathrm{H} & 6.0553459025 & -0.0184172240 & 4.2611796203 \\ \mathrm{H} & 7.5842691126 & -1.9519084637 & 4.3752529944 \\ \mathrm{H} & 6.7426823914 & -4.2474648000 & 3.9158399978 \\ \mathrm{H} & 4.3530127745 & -4.5843227708 & 3.2901030321 \\ \mathrm{H} & 2.8260564258 & -2.6415200300 & 3.1432879598 \\ \mathrm{C} & 4.7099932797 & 2.1336833010 & 4.9167192024 \\ \mathrm{C} & 5.3504079600 & 3.3729469907 & 4.9674926605 \\ \mathrm{C} & 5.2383376724 & 4.2664569310 & 3.8962149642 \\ \mathrm{C} & 4.4787523146 & 3.9175162943 & 2.7770035204 \\ \mathrm{C} & 3.8430535635 & 2.6731034707 & 2.7215197865 \\ \mathrm{H} & 4.7750459036 & 1.4599825625 & 5.7663642806 \\ \mathrm{H} & 5.9265513114 & 3.6451105457 & 5.8480256244 \\ \mathrm{H} & 5.7319706937 & 5.2336854838 & 3.9408890358 \\ \mathrm{H} & 4.3722277978 & 4.6096624491 & 1.9461612789 \\ \mathrm{H} & 3.2388542118 & 2.4119220368 & 1.8578263796 \\ \mathrm{C} & 2.6649088419 & -0.9637041793 & 6.3051436438 \\ \mathrm{C} & 2.1120447787 & -0.9681860906 & 7.5902354775 \\ \mathrm{C} & 1.2560502890 & 0.0583939726 & 7.9915698560 \\ \mathrm{C} & 0.9512816311 & 1.0948029826 & 7.1021525603 \\ \mathrm{C} & 1.4999068364 & 1.1026574777 & 5.8211743865 \\ & & & \\ & & & \end{array}$




$\begin{array}{lrr}3.3303225959 & -1.7672879657 & 6.0088453352 \\ 2.3552761722 & -1.7775464695 & 8.2738537366 \\ 0.8240374708 & 0.0512289982 & 8.9889117687 \\ 0.2788329078 & 1.8940225430 & 7.4017259403 \\ 1.2423406059 & 1.9016362978 & 5.1337007397 \\ 3.4246415144 & -0.0918873426 & -0.0424883480 \\ 5.2185672279 & -0.4713829977 & 0.2547513446 \\ 2.9264854552 & -1.4276556828 & -1.2145928922 \\ 3.5318493185 & 1.4369305497 & -1.0844972802 \\ 5.7577685659 & -1.7387304999 & -0.0165669340 \\ 7.1102550748 & -2.0036881494 & 0.2212398681 \\ 7.9459684611 & -1.0072684310 & 0.7299658630 \\ 7.4169350502 & 0.2557411016 & 1.0152146443 \\ 6.0651250272 & 0.5187230547 & 0.7881295720 \\ 5.1252269465 & -2.5191316986 & -0.4269943277 \\ 7.5085354073 & -2.9907692951 & -0.0004343966 \\ 8.9995025226 & -1.2117582644 & 0.9039117651 \\ 8.0556642408 & 1.0401795443 & 1.4140554119 \\ 5.6742059673 & 1.5065854464 & 1.0115726062 \\ 3.3076027330 & -1.4196757237 & -2.5656644433 \\ 2.9257182309 & -2.4629304693 & -3.4117840736 \\ 2.1653827012 & -3.5270457009 & -2.9149987434 \\ 1.7805225117 & -3.5393131137 & -1.5714385270 \\ 2.1484522495 & -2.4889115281 & -0.7277113273 \\ 3.8928859651 & -0.5950709908 & -2.9625288315 \\ 3.2199231153 & -2.4417218139 & -4.4584788673 \\ 1.8668902129 & -4.3378907463 & -3.5746819135 \\ 1.1752046628 & -4.3542512360 & -1.1820798538 \\ 1.8128392759 & -2.4797588092 & 0.3047260011 \\ 4.6715423800 & 1.7449092674 & -1.8501031605 \\ 4.7095731611 & 2.8986470648 & -2.6363736940 \\ 3.6109009634 & 3.7625582499 & -2.6675933410 \\ 2.4741632062 & 3.4633517611 & -1.9116860655 \\ 2.4300243374 & 2.3090313789 & -1.1244120168 \\ 5.5362432798 & 1.0895385040 & -1.8278347483 \\ 5.5987388344 & 3.1198327466 & -3.2217047311 \\ 3.6414929272 & 4.6617903568 & -3.2776794406 \\ 1.6122387479 & 4.1251004323 & -1.9351209958 \\ 1.5308989830 & 2.0642355662 & -0.5675787927\end{array}$




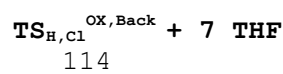

114

Geometry: 94 Total energy: $\quad-3592.33223311418$

C $\quad 5.1630238825 \quad-3.1007785853 \quad-8.6247063295$

$\begin{array}{llll}\mathrm{C} & 5.6603259817 & -1.8005064594 & -8.4141523589\end{array}$

C $\quad 6.4431325159-1.5436686312 \quad-7.2870853300$

C $\quad 6.7286304012-2.5599459062 \quad-6.3656281463$

$\mathrm{Cl} \quad 7.3303551969-5.3559151268 \quad-5.8443706569$

$\mathrm{H} \quad 5.8709659000 \quad-4.7436655192 \quad-1.2528691407$

$\mathrm{H} \quad 2.4150432704 \quad-3.1341242870 \quad-5.8736697154$

$\mathrm{H} \quad 3.8901936012-3.9262673130 \quad-1.5766877280$

$-2.6667259760-1.5281264832$

$\mathrm{H} \quad 2.4371934979 \quad-5.2394711189 \quad-5.9528555340$

$\mathrm{H} \quad 5.0208327691-5.1212302568 \quad-7.8712907782$

$-3.3130615750 \quad-9.5024366399$

5.4528234875

6.8598231843

7. 3916118903

2.0493993774

2.9793452532

5.2151696349

5.3396720162

1.2292929669

$-2.1543009032$

$-0.2285169701$

0.1036469930

$-6.5814025761$

$-6.0812412294$

$-6.1757718144$

$-7.1866963411$

1.6003032320

$-3.3029189703$

$-1.9899557374$

$-1.6003176329$

$-2.0936035123$

$-0.8237153117$

$-1.7595200313$

$-1.3278396395$

$-1.8035557697$

$-2.3534468066$

2. 4870103647

3. 2579544841

2. 8764306374

2. 1355790860

3. 7271281965

1. 4227550838

0.6229452163

1. 8939725490

3.1800342261

4. 3375574130

$-3.4362820304$

2. 9494565331

$-2.5603509283$

$-0.9534182553$

$-0.5487409366$

$-2.1655690944$

$-0.8926077253$

0.2159701524

$-3.3915916732$

$-4.1877671565$

$-2.3419722292$

$-1.3461634524$

$-0.5213717878$

$-7.0101501620$

$-5.7676663205$

$-6.7117777546$

$-1.2107687075$

$-6.5227985000$

$-5.2063552215$

$-5.0678592542$

$-6.7532572196$

$-2.0236822228$

$-2.1217303477$

$-2.1179596706$

$-1.0112762025$

$-0.5508906876$

$-2.3798014191$

$-4.2454103095$

$-4.2078084026$

$-3.8707649144$

$-4.1058838332$

$-3.9632002608$

$-5.9598717614$

$-5.9556608457$

$-5.3900334259$

$-6.3317851341$

$-5.2783728840$

$-3.9755657498$

$-4.3379749255$

$-9.0216571272$

$-2.7734762446$

$-2.0993152821$

$-1.2794904957$

$-2.1929985318$

$-7.7456902518$

$-5.7959511013$

$-6.3045313395$

$-6.0148378974$

$-7.3805885507$

$-8.6257371563$

$-7.3839918138$

$-7.3252359248$

$-6.5298072275$

$-7.1599196198$

$-10.0986739112$

$-8.5151200196$

$-8.4083309715$

$-9.4389790216$

$-7.5058680365$

$-7.4620851155$

$-6.4606660913$

$-5.2248935010$

$-5.7064522894$

$-5.6114207122$

$-6.2500794703$

$-8.3167702609$

$-7.7876611423$

$-3.8004424911$

$-2.2108262464$

$-1.6673211159$

$-2.9687155671$

$-1.1102433215$

$-7.2259010091$

$-6.6476648014$

$-4.2618322558$

$-2.8471518276$

$-3.1328265501$

$-3.7021352839$

$-5.4814079823$

$-5.2418070666$

$-4.7967811580$

$-0.3131422166$

$-1.4905901326$

$-9.1325076500$

$-7.1252147748$

$-5.5250608085$

$-4.0574973453$

$-5.1363606088$

$-2.1613964658$

$-4.6060288961$

$-1.3287411192$

3.1396680259

1. 6774659125

3.0583084533

$-3.6924274801$

$-4.6880200958$

$-3.8586133890$

$-2.7351423869$

$-1.2794007986$

$-0.8780972828$

$-0.4434630879$

$-1.6851841064$

$-2.8073864110$

$-4.0743437503$

1. 6535053183

$-4.1990459594$

$-2.7650528488$

$-2.3262749800$

$-0.0825622547$

$-0.6064597215$

$-2.1057233411$

$-2.2643435246$

$-2.7752630552$

$-1.3666830468$

$-1.2344694692$

0.8119203210

0.1620511403

$-0.4647573467$

$-2.5131965398$

$-0.1083015262$

$-2.7091154130$

$-2.1458032824$

$-4.5320401521$

$-4.9088769176$

$-5.0086262126$

$-3.7237428763$

$-0.5008278077$

$-0.5440673852$

$-3.7367822122$

$-3.0170024763$

$-1.7445274718$

$-4.1573372833$

$-3.0159671678$

$-1.7447646729$

$-0.2634758814$

$-4.4655454412$

$-3.4316283571$

$-5.0490198905$

$-5.5535856115$

1.2899482663

$-1.7075594068$

0.4585296081

$-1.2615944224$ 


$\begin{array}{rrr}-8.0826361148 & -3.7072277476 & -2.7306719102 \\ -2.8884235993 & -5.2013835366 & 3.4527314943 \\ -2.5890264576 & -6.9478169923 & 3.3481533267 \\ 1.0418047735 & -5.7649959657 & 3.4814890541 \\ 0.1512906726 & -4.2892893406 & 3.0395507306 \\ 0.2675814416 & -5.4284070903 & 0.8564227046 \\ 0.0406519909 & -7.0194723110 & 1.6330198217 \\ -2.2597874104 & -6.5234864538 & 1.0056506045 \\ -0.9588927696 & -5.8328215656 & 3.9148857537 \\ 2.3215808710 & -8.6067036600 & -2.4338986383 \\ -7.6198000382 & -5.6817003588 & -2.9689209916 \\ -1.6811990713 & -8.3817811241 & -3.1085284335 \\ -3.3054222757 & -2.7962308815 & -2.3151619275 \\ 0.7893310775 & -1.2942589111 & 2.2555317473 \\ 2.9884021781 & -1.9773100190 & 1.5780919072 \\ 2.8862173773 & -1.9017759797 & 3.1021632051 \\ 1.8234946610 & -1.0773371451 & 1.1343646409 \\ 0.2832478946 & -0.3620101336 & 2.5452653370 \\ 0.0240954593 & -2.0247397172 & 1.9681664519 \\ 3.2916210324 & -2.7792826812 & 3.6194229559 \\ 3.4067335219 & -1.0070856495 & 3.4896354455 \\ 3.9601027398 & -1.6418694924 & 1.1994671630 \\ 2.8124738441 & -2.9961659557 & 1.2175162105 \\ 1.4448059704 & -1.3773827682 & 0.1535879902 \\ 1.4908106598 & -1.8248534463 & 3.3945620204 \\ 2.1350594243 & -0.0252474566 & 1.0940457829 \\ -3.4915342827 & -10.6889650694 & -7.2314219110 \\ -4.4013597916 & -9.4818145256 & -7.4808593446 \\ -4.3835041967 & -8.7991988669 & -6.0979717409 \\ -4.1128645241 & -9.9730399170 & -5.1187987619 \\ -3.6554479770 & -11.5247577218 & -7.9206290273 \\ -2.4286570520 & -10.3935751634 & -7.2931973048 \\ -3.2717112534 & -9.7491303244 & -4.4505088258 \\ -5.3185659084 & -8.2751926185 & -5.8762356168 \\ -3.5737047542 & -8.0633331462 & -6.0431434838 \\ -4.0496024948 & -8.8320384975 & -8.2896472068 \\ -5.4119789421 & -9.8260035489 & -7.7313972310 \\ -4.9872013349 & -10.2188532027 & -4.5045201232 \\ -3.8268990842 & -11.1303030482 & -5.9248081026 \\ 6.7349185952 & -4.5937506383 & -6.1906956052\end{array}$

